\title{
A Linear Accelerator Bunch Length Monitor
}

\author{
A Thesis \\ Submitted to the College of Graduate Studies and Research \\ in Partial Fulfillment of the Requirements \\ for the Degree of Master of Science in Engineering Physics \\ in the \\ Department of Physics and \\ Engineering Physics \\ University of Saskatchewan
}

\author{
by \\ Darryl Sheldon Jessie \\ Saskatoon, Saskatchewan \\ CANADA
}

Fall, 1995

(c) 1995 D. S. Jessie. All rights reserved. 
In presenting this thesis in partial fulfilment of the requirements for a Postgraduate degree from the University of Saskatchewan, the author agrees that the Libraries of this University may make it freely available for inspection. The author further agrees that permission for copying of this thesis in any manner, in whole or in part, for scholarly purposes may be granted by the professor or professors who supervised the thesis work or, in their absence, by the Head of the Department or the Dean of the College in which the thesis work was done. It is understood that any copying or publication or use of this thesis or parts thereof for financial gain shall not be allowed without the author's written permission. It is also understood that due recognition shall be given to the author and to the University of Saskatchewan in any scholarly use which may be made of any material in the thesis.

Requests for permission to copy or make other use of material in this thesis in whole or part should be addressed to:

\author{
Head of the Department of Physics and Engineering Physics \\ University of Saskatchewan \\ Saskatoon, Saskatchewan S7N 0W0
}




\begin{abstract}
The Saskatchewan Accelerator Laboratory is a $300 \mathrm{MeV}$ electron accelerator (Linac) and storage ring. Electrons are injected into the Linac in packets called bunches for acceleration by an electromagnetic field. The length of these bunches have a direct impact on the energy spread of the beam at the exit of the Linac. A large bunch length increases the background radiation in and around the Energy Compression System located at the end of the Linac and is an inefficient way of operating the accelerator.

Bunch length is dependent on the relative phase of the microwave power used by the Linac components. A monitor has been developed that will measure the bunch length early in injection so that the relative phase of the microwave power can be adjusted for optimal bunching.

The power available at two sufficiently separate harmonics in the frequency domain will give an indication of the bunch length in time. Simulations indicate the first and fourth harmonics fulfill this criteria.

The bunch length monitor consists of a circular disk of copper $1 \mathrm{~cm}$ in diameter located $3 \mathrm{~mm}$ from the inside surface of the beam pipe wall. It also includes a power splitter and two bandpass filters, one at the Linac RF frequency of $2.856 \mathrm{GHz}$ and the other at the fourth harmonic, $11.424 \mathrm{GHz}$.

The bunch length monitor was tested in a number of trials. By varying the prebuncher phase, two minima in bunch length were observed; one minima due to the injector and the other to the first accelerating section. A reduction of $12 \%$ in energy spread was observed and it is anticipated that with more thorough optimization, a reduction of up to $20 \%$ could be realized.
\end{abstract}




\section{Acknowledgements}

I would like to thank my supervisor, Dr. Eric Norum, for his help and guidance in the research and preparation of this document. His uncanny insight and willingness to drop whatever he was doing to answer my most trivial questions is greatly appreciated. It is my hope that some of his superb engineering ability has been incorporated into this project.

The financial support that Dr. D. M. Skopik has made available is greatly appreciated. Many thanks to Dr. H. S. Caplan for kindling my interest in obtaining a graduate degree.

The knowledge and assistance of all the SAL staff, past and present, was very welcome. In particular Don Cruickshank's experience in microwave circuits and construction made difficult problems easy. I am also grateful to Dr. Jim Murphy who on short notice would find some beam time for something I just had to check out.

My fellow graduate students have made SAL a great place to make new friends. In particular, Don Tiller, Dave Hornidge and Keith Helgason have made some long winter months a lot of fun.

I appreciate the support my Father and Mother, Dallas and Dorothy, have given me over the years. Their enthusiasm and interest in my University career surpassed mine on many occasions.

Finally, I thank my wife, Julie, for her understanding and cheerfulness through some difficult times. You were always willing to help in whatever capacity you could, and it is to you that I dedicate my thesis. 


\section{Contents}

1 Introduction 1

1.1 Saskatchewan Accelerator Laboratory . . . . . . . . . . . . . . 1

1.1.1 Accelerator Front End ............... 3

1.1.2 The Transmitter ................. 4

1.1.3 The Injector ....................... 4

1.2 Monitor Overview . . . . . . . . . . . . . . . 7

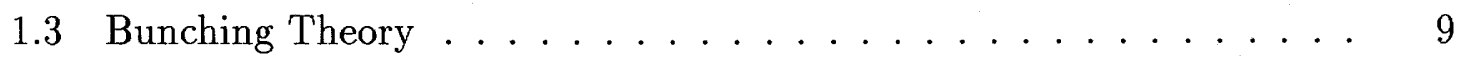

2 Theory 16

2.1 Fourier Spectral Components . . . . . . . . . . . . 16

2.1.1 Fourier Analysis of a Gaussian Distribution . . . . . . . 16

2.1.2 Fourier Analysis of a Differentiated Gaussian . . . . . . . . . . 19

2.2 Theoretical Output . . . . . . . . . . . . . . . 20

2.2.1 Equivalent Circuit ................ 20

2.2 .2 Button Impedance .................. 24

3 Filter Simulations $\quad 25$

3.1 Transfer Function ..................... 25

3.2 Digital Simulation. . . . . . . . . . . . . . . 29

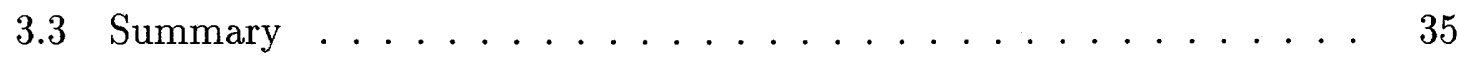

4 The SAL Bunch Length Monitor 36

4.1 The Bench Test . . . . . . . . . . . . . . 36

4.1.1 Component Characterization . . . . . . . . . . . 36

4.1.2 The Bench Measurements ............. . 41

4.2 Experimental Beam Trials . . . . . . . . . . . . . . 45 
$4.2 .1 \quad$ April Run $\ldots \ldots \ldots \ldots \ldots \ldots \ldots \ldots$

4.2 .2 July Run . . . . . . . . . . . . . . . . . 49

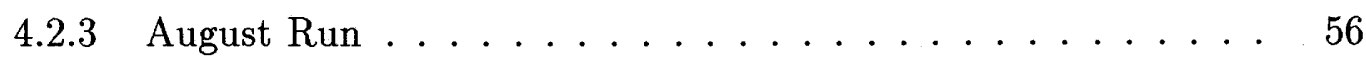

5 Summary and Conclusions $\quad 59$

$\begin{array}{ll}\text { A Filter Specifications } & 63\end{array}$

B Computer Programs $\quad 64$

$\begin{array}{ll}\text { C Bench Test Results } & 79\end{array}$

D April Results with Peak Power Meter $\quad 85$

E April Results with Crystal Detectors 91

$\begin{array}{ll}\text { References } & 94\end{array}$ 


\section{List of Tables}

$1.1 \quad$ SAL $\operatorname{Linac} \ldots \ldots \ldots \ldots \ldots \ldots \ldots \ldots \ldots$

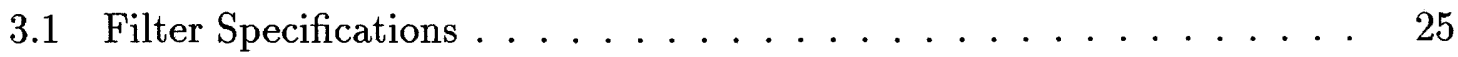

3.2 Frequency Numbers . . . . . . . . . . . . . . . . 26

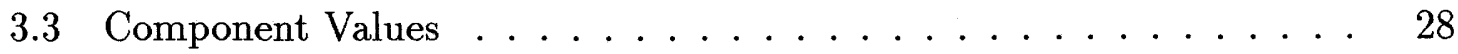

3.4 Coefficients for Discrete Equation . . . . . . . . . . . . 31

3.5 Theoretical, Simulated and Experimental Values . . . . . . . . . 33

3.6 Output Values for Differentiated Gaussian . . . . . . . . . . 35

4.1 Summary of Button Outputs . . . . . . . . . . . . . 44

4.2 Summary of Button Output for April Test Run . . . . . . . . . 47

4.3 Summary of Button Output from Crystal Detectors . . . . . . . . 49

4.4 Summary of Button Output for July Test Run . . . . . . . . . . 53

4.5 Energy Spread of Beam . . . . . . . . . . . . . . . . 54

4.6 Summary of Button Output for August Run, Section \#1 Phase $69^{\circ}$. 56

4.7 Summary of Button Output for August Run, Buncher Phase $75^{\circ} \ldots 57$

5.1 Energy Spread of Beam . . . . . . . . . . . . . . . 60 


\section{List of Figures}

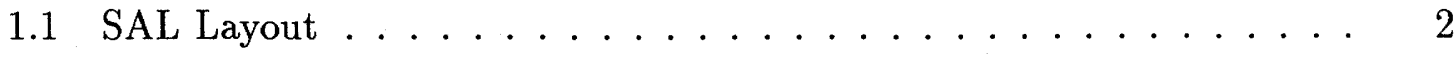

1.2 Injector Components $\ldots \ldots \ldots \ldots \ldots \ldots \ldots$

1.3 Pulse Relationships in Time: a) Gun Pulse Length, b) Bunch Period and Bunch Width, c) RF Pulse Width and Pulse Repetition Period . 6

1.4 Electron Variations before Buncher: a) DC Beam, b) End of Prebuncher, c) End of Drift Space . . . . . . . . . . . . 10

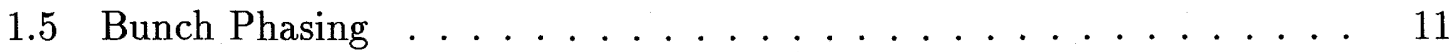

1.6 Theoretical Dependence of Bunch Width and Relative Phase Energy Spread . . . . . . . . . . . . . . . . . . 13

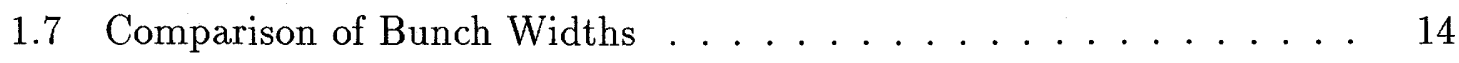

1.8 Theoretical Dependence of Bunch Width on Prebuncher and Section $\# 1$ Phase . . . . . . . . . . . . . . . . 15

2.1 Gaussian Wavetrain . . . . . . . . . . . . . . 16

2.2 Fourier Harmonic Magnitudes . . . . . . . . . . . . . . . 18

2.3 Fourier Harmonic Amplitudes for Differentiated Gaussian . . . . . . 20

2.4 Button Orientation . . . . . . . . . . . . . . . . . 21

2.5 Circuit Model . . . . . . . . . . . . . . . . . . 21

2.6 Differentiated Gaussian Wavepackets . . . . . . . . . . . 23

3.1 Desired Frequency Response . . . . . . . . . . . . . . . . 26

3.2 Low Pass Filter . . . . . . . . . . . . . . . . . . 27

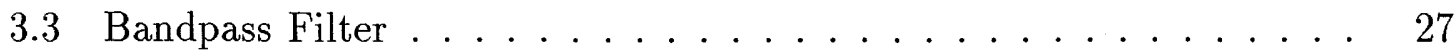

3.4 Bandpass Filter Response for First Harmonic . . . . . . . . . . . 29

3.5 Bandpass Filter Response for Fourth Harmonic . . . . . . . . . . 29

3.6 First Harmonic Response . . . . . . . . . . . . . . . . . . . 32

3.7 Fourth Harmonic Response . . . . . . . . . . . . . . . . . . 32 
3.8 First Harmonic Filter Response for $\sigma=8.0$ ps . . . . . . . . . . 34

3.9 Fourth Harmonic Filter Response for $\sigma=8.0$ ps . . . . . . . . . 34

4.1 Bunch Length Monitor with Test Fixtures . . . . . . . . . . . . 37

4.2 Theoretical and Experimental First Harmonic Response . . . . . . . 38

4.3 Theoretical and Experimental Fourth Harmonic Response . . . . . . 38

4.4 Response of Detector for First Harmonic . . . . . . . . . . . . . 40

4.5 Response of Detector for Fourth Harmonic . . . . . . . . . . . . . . . 40

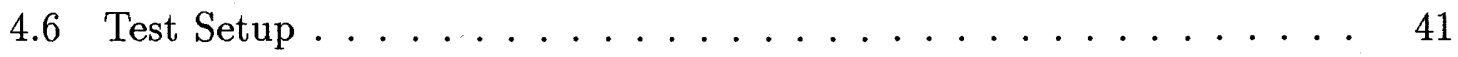

4.7 Switch Circuit . . . . . . . . . . . . . . . . 42

4.8 SRD Circuit . . . . . . . . . . . . . . . 43

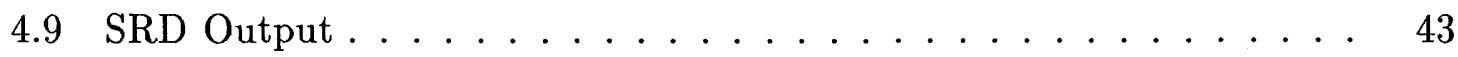

4.10 Experimental Test Setup . . . . . . . . . . . . . . . 46

4.11 April Results:Experimental Dependence of Prebuncher Phase on Bunch Width . . . . . . . . . . . . . . 48

4.12 Output of Monitor after Noise Subtraction . . . . . . . . . . . 49

4.13 April Result: First Harmonic Response with Prebuncher Phase 29 ${ }^{\circ}$. 50

4.14 April Result: Fourth Harmonic Response with Prebuncher Phase 29 50

4.15 July Result: First Harmonic Response with Prebuncher Phase 29 . . 51

4.16 July Result: Fourth Harmonic Response with Prebuncher Phase 29 ${ }^{\circ}$. 51

4.17 Components Between Section \#1 and Section \#2 . . . . . . . . 52

4.18 July Results: Experimental Dependence of Bunch Width on Pre-

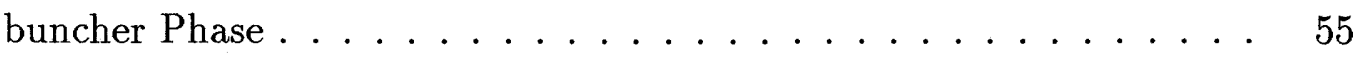

4.19 July Results: Experimental Dependence of Beam Energy on Pre-

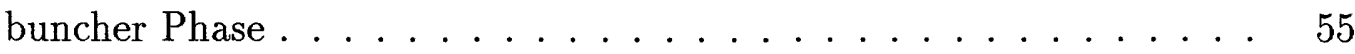

4.20 August Results:Experimental Dependence of Prebuncher Phase on Bunch Width for Section \#1 Phase of $69^{\circ}$. . . . . . . . . . . 58

4.21 August Results:Experimental Dependence of Prebuncher Phase on Bunch Width for Section \#1 Phase of $75^{\circ} \ldots \ldots . \ldots 58$ 
5.1 July Results: Experimental Dependence of Bunch Width on Prebuncher Phase . . . . . . . . . . . . . . . . . . 62

5.2 July Results: Experimental Dependence of Beam Energy on Prebuncher Phase . . . . . . . . . . . . . . . . . 62

A.1 Response Curve for Various Filters $\ldots \ldots \ldots \ldots \ldots$

D.1 $2856 \mathrm{MHz}:$ Prebuncher Phase $29^{\circ} \ldots \ldots \ldots \ldots$

D.2 $11424 \mathrm{MHz}:$ Prebuncher Phase $29^{\circ} \ldots \ldots \ldots \ldots$

D.3 $2856 \mathrm{MHz}$ : Prebuncher Phase $19^{\circ} \ldots \ldots \ldots \ldots \ldots$

D.4 $11424 \mathrm{MHz}$ : Prebuncher Phase $19^{\circ} \ldots \ldots \ldots \ldots \ldots$

D.5 $2856 \mathrm{MHz}$ : Prebuncher Phase $10^{\circ} \ldots \ldots \ldots \ldots \ldots$

D.6 $11424 \mathrm{MHz}:$ Prebuncher Phase $10^{\circ} \ldots \ldots \ldots \ldots \ldots$

D.7 2856 MHz: Prebuncher Phase $29^{\circ} \ldots \ldots \ldots \ldots \ldots \ldots$

D.8 $11424 \mathrm{MHz}:$ Prebuncher Phase $29^{\circ} \ldots \ldots \ldots \ldots \ldots \ldots$

D.9 $2856 \mathrm{MHz}:$ Prebuncher Phase $39^{\circ} \ldots \ldots \ldots \ldots \ldots$

D.10 $11424 \mathrm{MHz}:$ Prebuncher Phase $39^{\circ} \ldots \ldots \ldots \ldots \ldots$. . . . 87

D.11 2856 MHz: Prebuncher Phase $49^{\circ} \ldots \ldots \ldots \ldots \ldots$

D.12 $11424 \mathrm{MHz}:$ Prebuncher Phase $49^{\circ} \ldots \ldots \ldots \ldots \ldots$

D.13 2856 MHz: Prebuncher Phase $59^{\circ} \ldots \ldots \ldots \ldots \ldots$

D.14 11424 MHz: Prebuncher Phase $59^{\circ} \ldots \ldots \ldots \ldots$. . . . . . 88

D.15 2856 MHz: Prebuncher Phase $69^{\circ} \ldots \ldots \ldots \ldots$. . . . . . 89

D.16 $11424 \mathrm{MHz}$ : Prebuncher Phase $69^{\circ} \ldots \ldots \ldots \ldots$

D.17 2856 MHz: Prebuncher Phase $79^{\circ} \ldots \ldots \ldots \ldots$. . . . . 89

D.18 11424 MHz: Prebuncher Phase $79^{\circ} \ldots \ldots \ldots \ldots$. . . . . . . 89

D.19 2856 MHz: Prebuncher Phase $29^{\circ} \ldots \ldots \ldots \ldots \ldots$

D.20 11424 MHz: Prebuncher Phase $29^{\circ} \ldots \ldots \ldots \ldots \ldots$

E.1 Raw $2856 \mathrm{MHz}$ Signal . . . . . . . . . . . . . . . . 91

E.2 Raw $11424 \mathrm{MHz}$ Signal . . . . . . . . . . . . . . . . . . . 92 
E.3 Output of Monitor after Noise Subtraction . . . . . . . . . . . . 92

E.4 Prebuncher Phase $9^{\circ} \ldots \ldots \ldots$. . . . . . . . . . . . 93

E.5 Prebuncher Phase $49^{\circ} \ldots \ldots \ldots$. . . . . . . . . . . . 93

E.6 Prebuncher Phase $69^{\circ} \ldots \ldots \ldots . \ldots \ldots$ 


\section{Chapter 1}

\section{Introduction}

The bunch length monitor described in this thesis provides a means of determining the bunching efficiency with the accelerator in operation. An accelerated electron beam has an energy spread. The deviation from the monoenergetic ideal is caused by perturbations in beam acceleration, with the greatest effect due to the relative RF phasing of the injector components.

The injector is located at the front end of the linear accelerator (Linac) section of SAL. Immediately after the Linac is the Energy Compression System (ECS). If the injector component phasing is set to provide a broad energy spectrum, the energy defining slits located in the ECS will physically remove the energy deviant electrons from the beam and ultimately reduce the available electrons exiting the ECS. This has the undesirable effect of decreasing available current after the ECS while increasing the background radiation in the Linac vault. The optimal operating condition is to have the injector provide the smallest energy spectrum possible, causing a maximum in available current and a minimum in ECS radiation. This optimization can be done by limiting the bunch length of the electron packets early in the acceleration process, since this sets the ultimate limit on the narrowness of the energy spectrum [6].

\subsection{Saskatchewan Accelerator Laboratory}

The Saskatchewan Accelerator Laboratory is a $300 \mathrm{MeV}$ electron accelerator and storage ring located on the University of Saskatchewan campus. Figure 1.1 illustrates the location of the bunch monitor with respect to other components. 


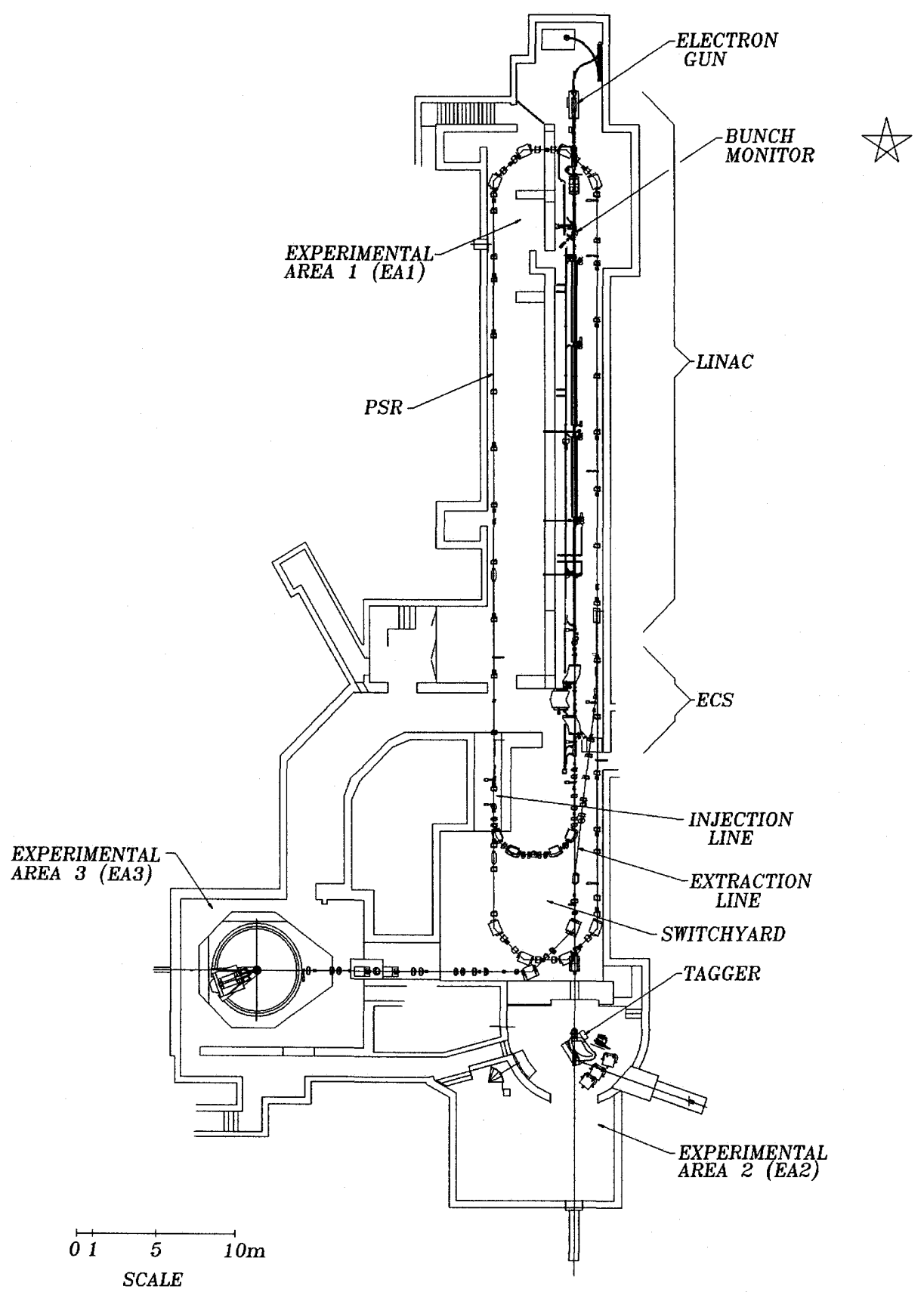

Figure 1.1: SAL Layout 


\subsubsection{Accelerator Front End}

The main elements of the Injector are illustrated in Figure 1.2. The different components are labelled and show relative orientation and structure. Oscilloscope displays are provided to give an intuitive idea of the pulse manipulation that occurs at each component. Brief descriptions of these elements will provide sufficient background for further discussion.

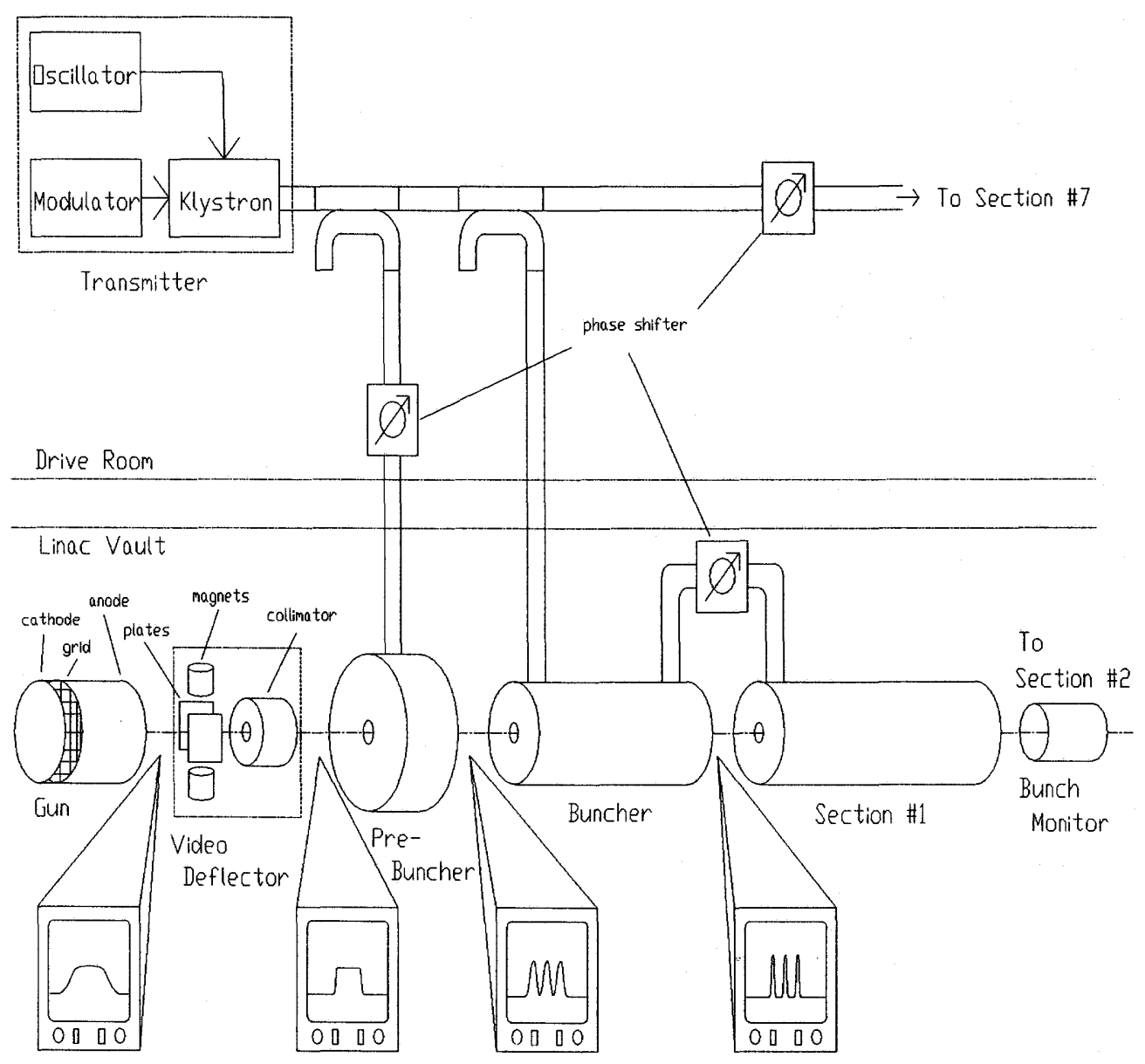

Figure 1.2: Injector Components 


\subsubsection{The Transmitter}

The transmitter provides the electromagnetic energy for acceleration of the beam. As displayed in Figure 1.2, it consists of a microwave oscillator, modulator and klystron.

The main components of the klystron include the electron gun, two RF resonant cavities, and a collector. The microwave oscillator provides a frequency of $2856 \mathrm{MHz}$ to the input cavity of the klystron. The cavity resonates at this frequency, and velocity modulates the DC beam supplied by the gun. The beam then travels through a drift space and into the output cavity, which couples the field of the bunched electron beam to waveguides for transport to the Linac beam line. The klystron beam is then dumped into the collector.

The klystron can supply brief pulses of about $20 \mathrm{MW}$ of microwave power at a rate of up to $360 \mathrm{~Hz}$. This rate is determined by the modulator trigger, which gates the klystron gun with pulses variable from 300 to $2200 \mathrm{~ns}$. The main elements of the modulator are the Pulse Forming Network (PFN) and the hydrogen thyratron. The PFN slowly stores energy in capacitors, that discharge rapidly when the thyratron is fired. The transmitter thus provides a rectangular pulse of microwave power to the Linac beam line.

This microwave power is then split for use in the prebuncher $(1 \mathrm{~kW})$, buncher $(13$ MW) and section seven (6+MW) which is an accelerating section located just after the ECS. The power left over from the buncher is phase-shifted to provide power to Section \#1 ( $\approx 9 \mathrm{MW})$.

\subsubsection{The Injector}

The injector consists of the gun, video deflector, prebuncher and buncher and are illustrated in Figure 1.2.

The electron gun supplies electrons by thermionic emission. The gun is of a triode configuration which includes a cathode, grid and anode. Its operation depends on the potential of the grid relative to the cathode. The cathode potential is at $-220 \mathrm{kV}$ 
relative to the anode. When the grid potential is changed from $-225 \mathrm{kV}$ to $-220 \mathrm{kV}$, a square pulse with rising and trailing edges of $200 \mathrm{~ns}$ and a width that can be set up to $5000 \mathrm{~ns}$ is provided for acceleration.

The video deflector is used to sharpen the gun pulse edges. The deflector consists of a parallel plate capacitor, magnets and collimator. First, the beam proceeds between metal plates, which have a potential between them. Magnets produce a magnetic field between the plates and perpendicular to the electric field. When a START is sent, the electric and magnetic fields produce forces on the beam that exactly cancel. The beam can then pass directly through the collimator. When a STOP is sent, the electric field is reduced in magnitude, and the beam is deflected into the face of the collimator.

The prebuncher is a $10.5 \mathrm{~cm}$ long resonant cavity that velocity modulates the beam much like the input cavity of the klystron. It does this by having the sinusoidal input from the transmitter superimposed upon the DC pulse of the gun. After the drift space between the prebuncher and buncher, the electrons are forced into packets of roughly $75^{\circ}$ intervals of the RF phase $[3,6]$. Approximately $65 \%$ of the incoming electrons are captured in these intervals [12] with the rest drifting toward the beam pipe wall at ground potential.

The buncher is $1.6 \mathrm{~m}$ in length and has these parts: an input coupler, a tapered structure that is connected directly to a constant phase velocity section, and an output coupler to provide power to the next accelerating section. The input coupler accepts power from the waveguide in a rectangular $\mathrm{TE}_{10}$ mode and transforms it to a circular $\mathrm{TM}_{01}$ mode for acceleration. The tapered section limits the phase velocity for one wavelength of the input RF to allow for greater capture efficiency from the prebuncher. After the tapered section, the beam has several $\mathrm{MeV}$ of kinetic energy. The constant velocity section adds more energy to the beam, which exits the buncher at approximately $13 \mathrm{MeV}$ of energy and has a phase spread on the order of $20^{\circ}$. The output coupler then takes the $\mathrm{TM}_{01}$ mode and transforms it to the $\mathrm{TE}_{10}$ mode for waveguide transport to Section \#1. For comparison, the relationships are sketched 
in Figure 1.3 and are tabulated in Table 1.1.
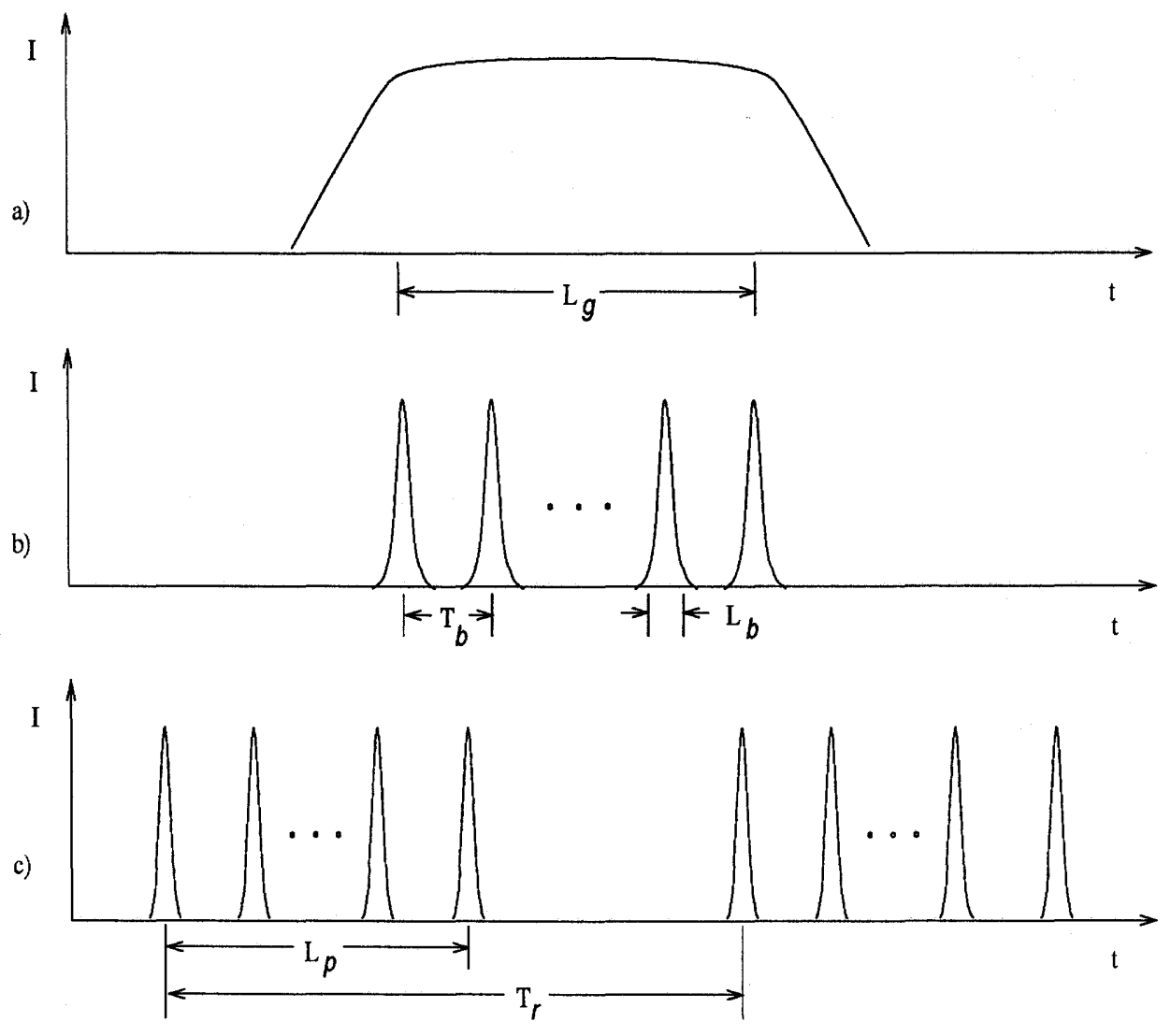

Figure 1.3: Pulse Relationships in Time: a) Gun Pulse Length, b) Bunch Period and Bunch Width, c) RF Pulse Width and Pulse Repetition Period

Table 1.1: SAL Linac

\begin{tabular}{|c|c|c|}
\hline Parameter & Symbol & Magnitude \\
\hline Gun pulse length & $\mathrm{L}_{g}$ & $5000 \mathrm{~ns}$ \\
\hline Bunch period & $\mathrm{T}_{b}$ & $350.14 \mathrm{ps}$ \\
\hline Bunch width & $\mathrm{L}_{b}$ & $\approx 20 \mathrm{ps}$ \\
\hline RF pulse width & $\mathrm{L}_{p}$ & $300 \rightarrow 2200 \mathrm{~ns}$ \\
\hline Pulse repetition period & $\mathrm{T}_{r}$ & $2.78 \rightarrow 500 \mathrm{~ms}$ \\
\hline
\end{tabular}

The beam is then accelerated through six sections and enters the ECS. The ECS provides a reduction in energy spread from $1 \%$ to $0.1 \%$ by the use of magnets, energy 
defining slits and Section \#7 [18]. The beam then enters the Electron Storage Ring of Saskatchewan (EROS) [8] for later experimental use.

\subsection{Monitor Overview}

The first step in the development of the SAL bunch monitor was to define the design requirements. It was found that five general specifications were needed to limit the information search for monitors that could be modified and used at SAL.

The most important specification was that the monitor should be non-intercepting. It is intended that monitoring occur during normal operation, which is an impossibility with an intercepting monitor. Fast and passive bunch measurement is also desirable; fast so that the bunch measurement can be done quickly and passive so that the beam is disturbed as little as possible. Mechanical simplicity is also a desirable trait, since a more complicated design is generally less reliable. Lastly, the least expensive monitor with the most accuracy is a fundamental objective.

A survey of previous monitor designs for bunch monitoring revealed some ingenious and effective methods. Below are some of the designs considered for the SAL bunch monitor.

One of the more accurate methods was that designed by J. C. Sheppard [26] for the Stanford Linear Collider Linac. The main components include a vacuum vessel containing a quartz radiator and window, a streak camera and analyzer electronics. The beam enters the vacuum vessel and strikes a quartz radiator. This produces Cerenkov light, which passes through a window to a streak camera. The analyzer electronics include an oscilloscope that provides bunch intensity as a function of time. Although a resolution of $2.4 \mathrm{ps}$ for a bunch length of $\approx 10 \mathrm{ps}$ is impressive, this method intercepts the beam and is also a complicated monitor.

A stripline monitor is a metallic plate close to the inside surface of the beam pipe

wall. It has coaxial terminations through vacuum sealed upstream and downstream ports. Stripline monitors have been used for bunch monitoring at many facilities 
[13]. These monitors are designed to detect two frequency harmonics of the revolving beam and take the ratio of their amplitudes. This type of monitor is simple and well understood but its capacitance is high, giving it lowpass characteristics. For our application, a bandwidth in the tens of gigahertz is required.

An absolute measurement of the bunch length can be done by using an RF sweeper. One plate is fed with RF from a klystron which also drives the injector. At the plate, the $\mathrm{RF}$ is changed in phase so that the beam is swept to allow passage through a slit. The beam is collected at the slit and a charge dependence per relative injector phase can be found $[3,6]$. This method provides an accurate result, but cannot be used for our purposes since it intercepts the beam for a short time.

A resonant cavity method was also considered. This had been done at the Stanford Linear Accelerator in 1966 [3]. It involves the beam travelling through a specially designed pillbox cavity with a geometry that insures resonance at two harmonics of the beam frequency. The fields inside the cavity are then coupled out and their ratio taken to give relative bunching efficiency. Although this provides a non-destructive measurement of the beam, the cavity must have very accurate dimensions which are immune to expansion and contraction due to changes in temperature.

The most frequently used monitor is the electrostatic pickup monitor [14, 25]. A disc shape electrode "button" is center terminated to coaxial cables outside the pipe. The button is situated close to the inside surface of the beam pipe, and provides a signal by sensing the disturbance caused by the electromagnetic field of the beam. This monitor is mechanically simple, passive and inexpensive. It does not intercept the beam and provides a quick measurement of relative bunching efficiency. There is also abundant literature on its principles of operation. For these reasons, this design was selected for the SAL bunch length monitor. 


\subsection{Bunching Theory}

To understand the bunching process, the prebuncher must be considered in more detail. As mentioned before, it is a pillbox cavity of $10.5 \mathrm{~cm}$ in length that operates in the $\mathrm{TM}_{010}$ mode. The $\mathrm{RF}$ drives the cavity via an $\mathrm{N}$ type coaxial loop. A phase shifter allows variation of the relative phase of the cavity with the buncher. An attenuator allows a range of power levels on either side of the $1 \mathrm{~kW}$ nominal operating value.

The purpose of the prebuncher is to prepare the beam so that as many electrons as possible can be accelerated in the buncher [28]. This is done by concentrating the particles at a particular phase in the buncher which will remain synchronous with the wave through acceleration. This synchronous phase is denoted by $\phi_{s}$.

As a continuous electron stream passes through the cavity, some electrons are accelerated and some decelerated. Since the electrons at this point are not relativistic, velocity modulation occurs and bunching is done mainly in the drift space between the prebuncher and buncher. The process follows the progression of Figure 1.4 where $\Delta E$ is the change in kinetic energy of the beam plotted as a function of the phase of the modulating field.

The electrons from the prebuncher are then captured by the buncher at a particular phase $\phi$. Consider a single electron entering the buncher. Since the buncher operates in a $\mathrm{TM}_{01}$ mode, only the axial $E_{z}$ field is of concern $[12,6,17]$. Figure 1.5 illustrates an axial electric field with the abscissa being the phase $\phi$ or $z$.

If the electron is located at $\phi_{s}$, it will travel with the wave at a constant phase. A particle slightly ahead of $\phi_{s}$ will see a weaker electric field magnitude and therefore a smaller force, causing the electron to be driven back into $\phi_{s}$. Conversely, if the particle is slightly behind $\phi_{s}$ it will have a stronger force directing it to the synchronous phase. For small and slow deviations in phase, an electron is trapped in a potential well and 

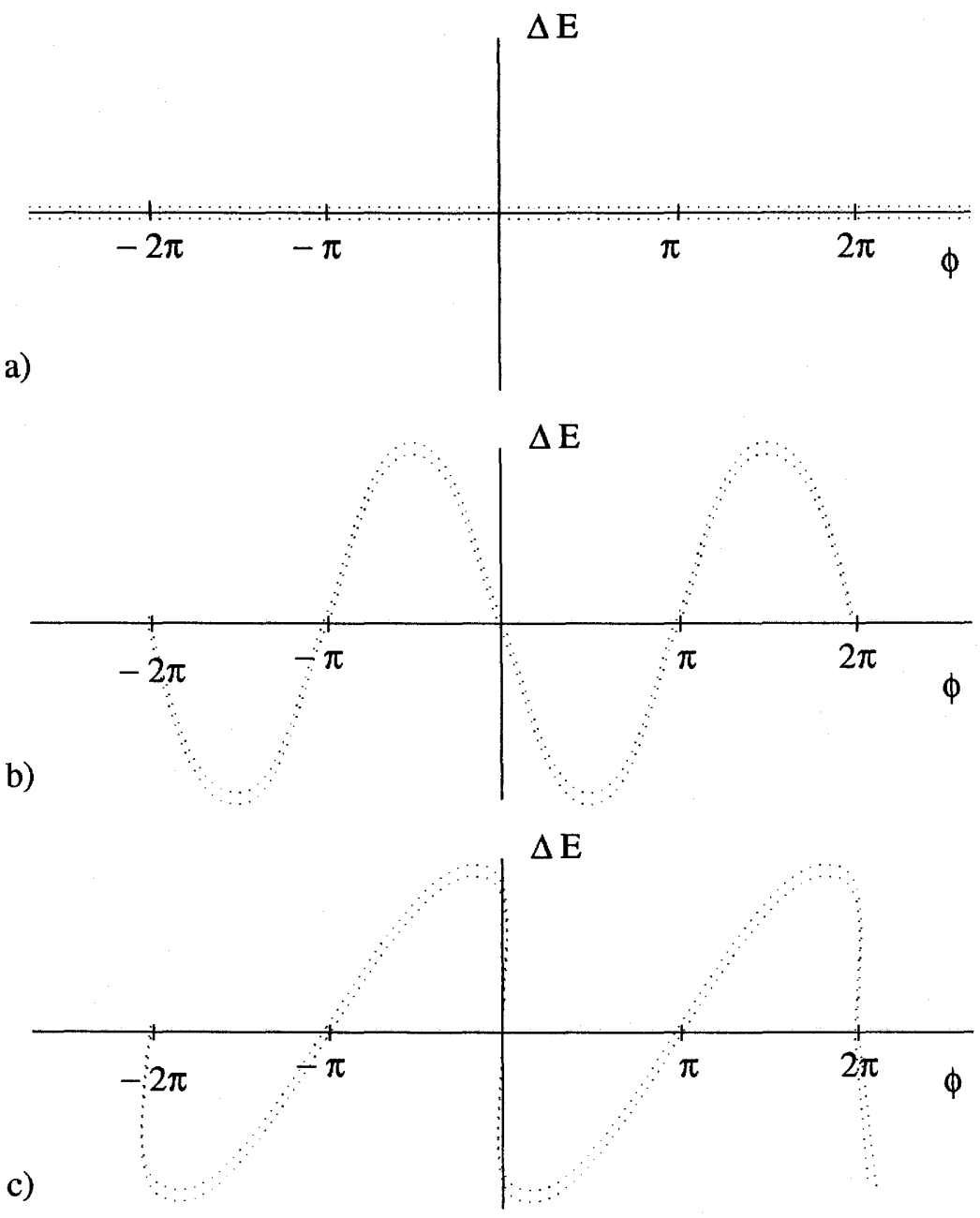

Figure 1.4: Electron Variations before Buncher: a) DC Beam, b) End of Prebuncher, c) End of Drift Space

will undergo synchronous oscillations with frequency [17]:

$$
\omega_{\phi}=\sqrt{\frac{\omega_{o} e E \sin \phi_{s}\left(1-\beta_{s}^{2}\right)^{3 / 2}}{v m_{0} \beta_{s}}}
$$

where $E$ is the electric field strength, $e$ is the electron charge, $\beta_{s}$ is the velocity of the synchronous particle normalized to the velocity of light $c$, and $\omega_{o}, m_{0}$ are the frequency of the RF and rest mass of the electron, respectively. From Equation 1.1, as the velocity of the electron becomes large these oscillations almost cease to occur. 


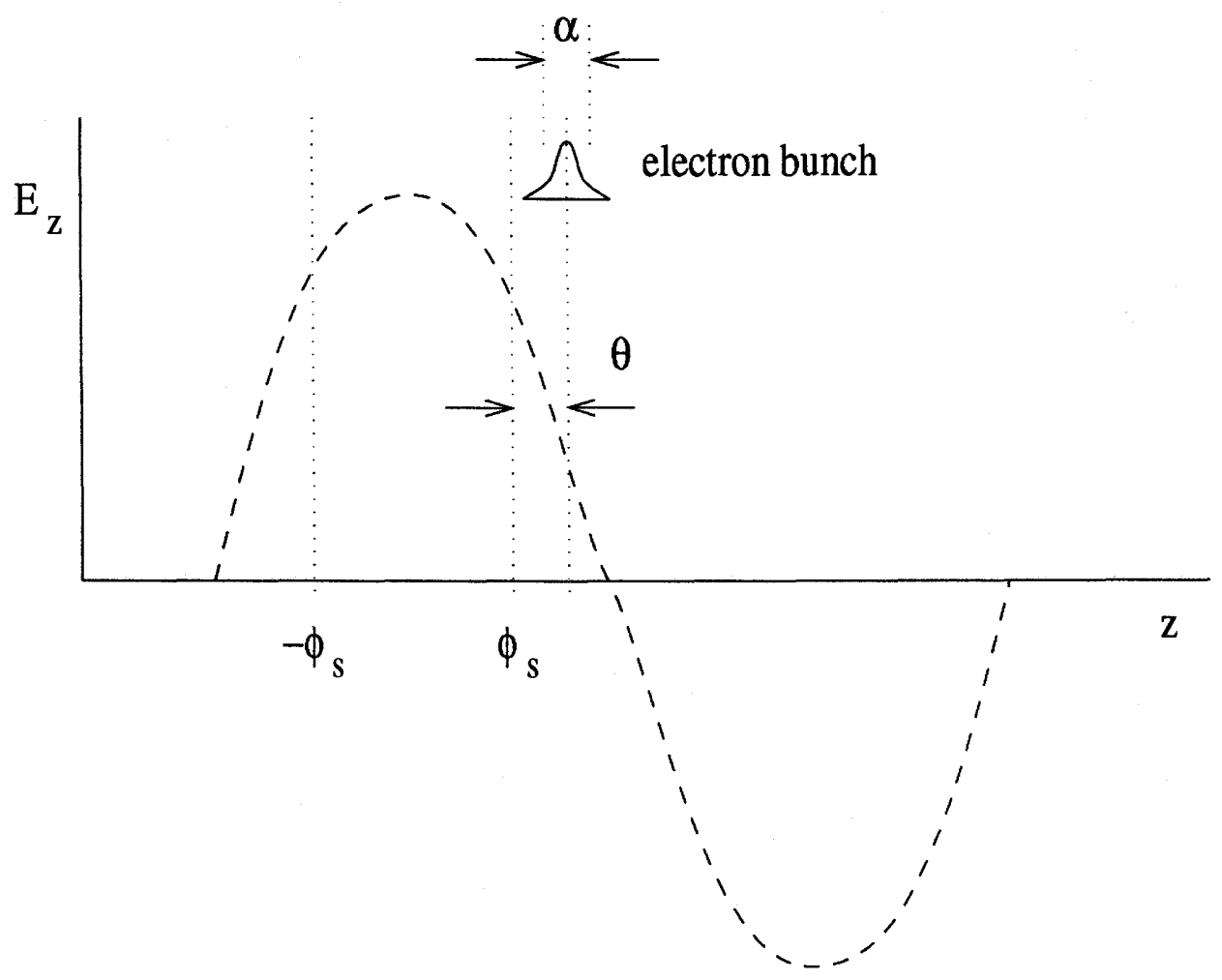

Figure 1.5: Bunch Phasing

The capture region of the buncher is defined as the limit in phase that an electron can deviate from $\phi_{s}$ and still be captured. If the electron is at a more negative phase than $-\phi_{s}$, this electron will drop even further back. This is the boundary on one side of the capture region. The boundary on the positive side has been shown by Kollath to be $\approx 2 \phi_{s}[17]$ giving a capture region of $\approx 3 \phi_{s}$. By inspection, a bunch with phase deviating far from the synchronous phase will have electrons drop out of the acceleration process.

The limit of phase deviation of the particles from $\phi_{s}$ due to the synchronous oscillations is given by [17]:

$$
\Delta \phi=\frac{A}{\beta_{s}} \sqrt[4]{\frac{2 \pi\left(1-\beta_{s}^{2}\right)^{3 / 2} \beta_{s}}{m_{0} \lambda_{0} e E \sin \phi_{s}}} \sin \omega_{\phi} t
$$

where $A$ is a constant. As the acceleration progresses, the electrons will have their os- 
cillations damped and the total bunch width will shrink. This equation, like Equation 1.1 , is only valid for small and slow variations in phase which Kollath approximates at energies below $0.5 \mathrm{MeV}$. This is sufficient, since this is where most of the bunching effect occurs.

Two cases of possible bunch injection will now be considered. If an electron bunch is injected into the buncher with the centroid of charge at $\phi_{s}$, the oscillations will damp out, the bunch will shrink and the ideal case has occurred. However, if the packet is injected into the buncher with the centroid away from $\phi_{s}$, many more particles will undergo oscillations. While the velocities increase and thus reduce the oscillations, the bunch will be smeared in phase about $\phi_{s}$. This is how the phase and ultimately the energy spread of the beam is increased [6].

Brown et al. [5] have shown that the energy spread of a linear accelerator beam after injection into an accelerating section is dependent on two parameters; the phase deviation of the bunch from the peak electric field and on the bunch width. This is given by:

$$
\delta V / V=1-\cos \left(\frac{\alpha}{2}\right)\left[1+\left(\frac{\sin (\theta)}{\cos (\theta)}\right)^{2}\right]^{-\frac{1}{2}}+\sin \left(\frac{\alpha}{2}\right)\left[1+\left(\frac{\cos (\theta)}{\sin (\theta)}\right)^{2}\right]^{-\frac{1}{2}}
$$

where $\theta$ is the phase deviation from the maximum at the wave crest and $\alpha$ is the bunch width. By plotting Equation 1.3 for a bunch width of $30^{\circ}$, the illustration given in Figure 1.6 is obtained. A clear minimum occurs about the zero phase position.

As will be discussed in Chapter 2, the button monitor provides a differentiated Gaussian voltage signal in the frequency domain for each harmonic of the machine frequency. To provide a look at the effect of moving away from a small bunch width, the first harmonic signal is divided by the fourth harmonic signal as shown in Equation 1.4

$$
\frac{V_{1}}{V_{4}}=0.25 e^{\frac{15 \omega_{0}^{2} \sigma^{2}}{2}}
$$

where $V_{1}$ is the voltage of the first harmonic signal and $V_{4}$ is the voltage at the 


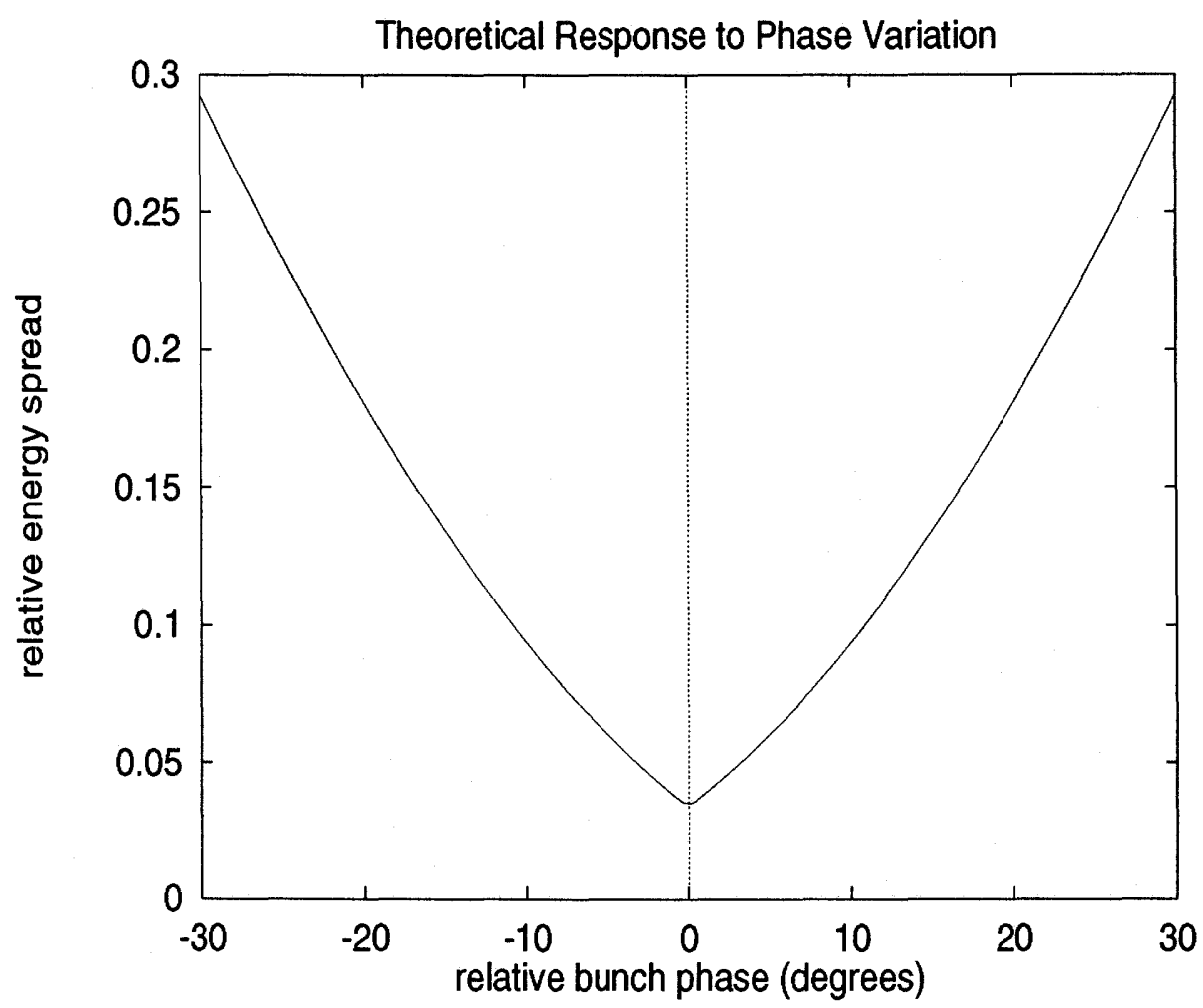

Figure 1.6: Theoretical Dependence of Bunch Width and Relative Phase Energy Spread

fourth. The variable $\omega_{o}$ is the Linac RF frequency and $\sigma$ is the standard deviation of a Gaussian pulse.

For application to the injector only, the indication is that an exponential dependence is expected for the button output as the prebuncher phase is changed from $\phi_{s}$. This dependence is assumed to be symmetrical for small deviations about the minimum bunch width and becomes approximately constant as the centroid of injected charge moves away from $\phi_{s}$ and approaches the peak of the sinusoidal field, as illustrated in Figure 1.7.

If the assumption is made that a change of one degree will vary the Gaussian $\sigma$ by one picosecond, the superposition Figures 1.6 and 1.7 yields Figure 1.8. This curve is obtained by arbitrarily selecting the minimum for the injector at $50^{\circ}$ and the minimum for Section \#1 at $20^{\circ}$. It is clear that two minima would occur over the 


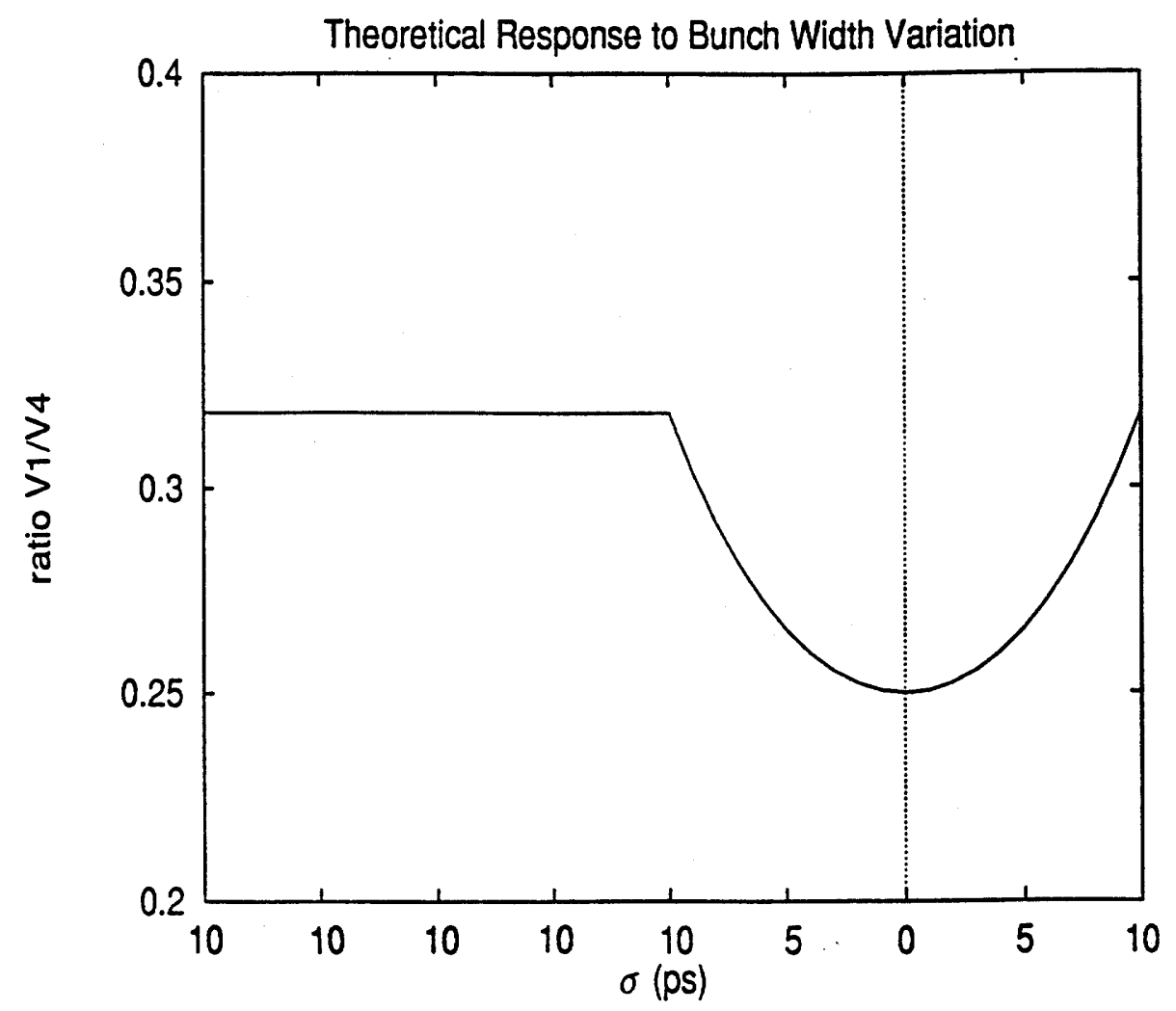

Figure 1.7: Comparison of Bunch Widths

range of the prebuncher phase; one due to the injector and the other to Section \#1 as demonstrated in Figure 1.8.

Bunch width is set at injection and remains constant throughout acceleration $[3,17]$, therefore microwave phasing at injection is important in limiting bunch length. This combined with the relative phasing of the bunches at entry to each section are major factors in limiting energy spread of the beam. 


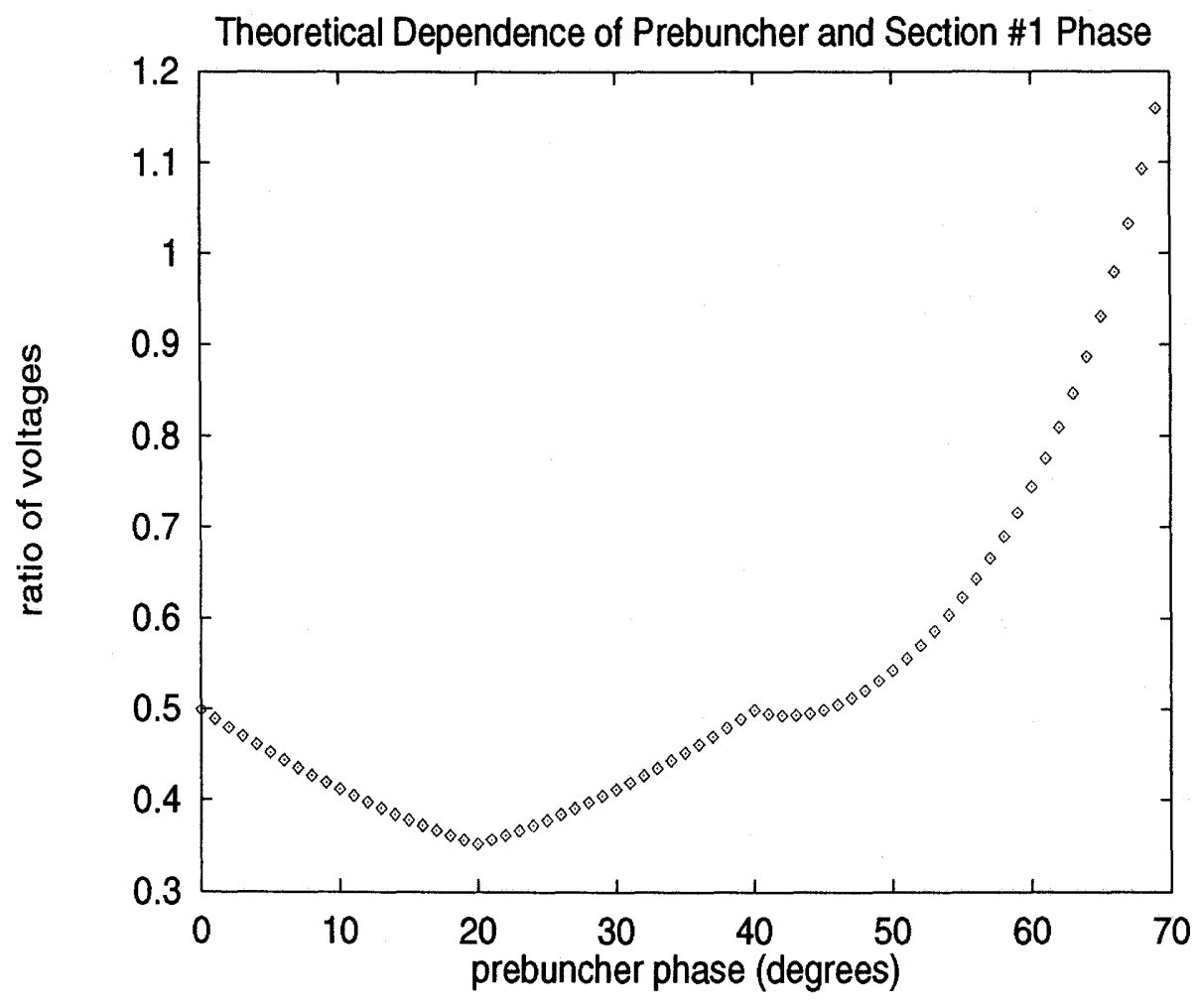

Figure 1.8: Theoretical Dependence of Bunch Width on Prebuncher and Section \#1 Phase 


\section{Chapter 2}

\section{Theory}

\subsection{Fourier Spectral Components}

The beam bunch monitor described in this thesis compares two components of the Fourier spectrum of the beam; the fundamental component which corresponds to the distances between bunches, and a higher harmonic which depends on the temporal shape of the beam [7]. The theoretical development leading to the magnitudes of these components will now be presented.

\subsubsection{Fourier Analysis of a Gaussian Distribution}

The derivation below has been done by Shafer [25]. The beam bunches are assumed to be Gaussian in current distribution as illustrated in Figure 2.1.

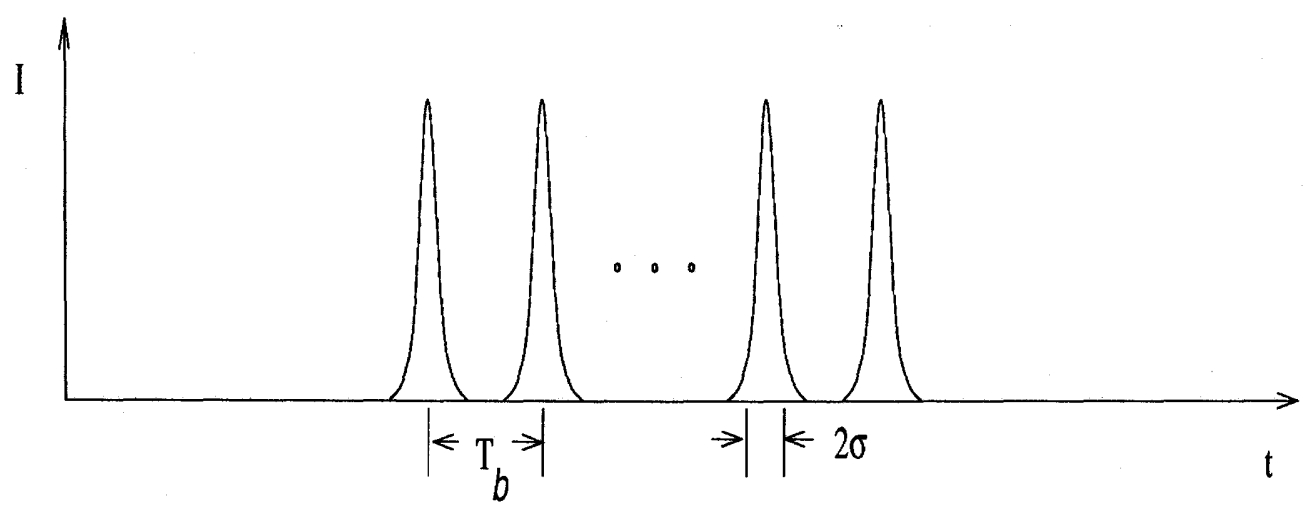

Figure 2.1: Gaussian Wavetrain

The charge packets can be described as travelling waves given by:

$$
I_{b}(z, t)=\frac{N e}{\sqrt{2 \pi} \sigma} \exp \left(-\frac{(v t-z)^{2}}{2 \sigma^{2}}\right)
$$


where $N e$ is the total charge in each bunch, $\sigma$ is the standard deviation of a Gaussian pulse and $v$ is the velocity of the bunch. In the time domain this is written:

$$
I_{b}(t)=\frac{N e}{\sqrt{2 \pi} \sigma}\left[\exp \left(\frac{-t^{2}}{2 \sigma^{2}}\right)+\exp \left(\frac{-\left(t-T_{b}\right)^{2}}{2 \sigma^{2}}\right)+\ldots\right]
$$

The period $T_{b}$ was defined in Table 1.1 and $t$ is the time that can vary from 0 to $+\infty$. The number of packets in the wavetrain is given by the ratio of RF pulse length and $\mathrm{RF}$ period, which is on the order of a few thousand. Therefore, the assumption is made that the wavetrain is continuous and periodic in the time domain. The Fourier series is then given by the Euler formula:

$$
f(t)=\frac{a_{0}}{2}+\sum_{n=1}^{\infty}\left(a_{n} \cos \frac{2 \pi n}{T} t+b_{n} \sin \frac{2 \pi n}{T} t\right)
$$

where $T$ is the period of the periodic waveform. The coefficients are defined as

$$
\begin{aligned}
& a_{n}=\frac{1}{T} \int_{\frac{-T}{2}}^{\frac{T}{2}} f(t) \cos \left(\frac{2 \pi n}{T} t\right) d t \\
& b_{n}=\frac{1}{T} \int_{\frac{-T}{2}}^{\frac{T}{2}} f(t) \sin \left(\frac{2 \pi n}{T} t\right) d t
\end{aligned}
$$

Since the Gaussian pulse is an even function, only the $a_{n}$ survive. Therefore:

$$
\begin{aligned}
& a_{0}=\frac{N e}{T} \\
& a_{n}=\frac{N e}{T \sqrt{2 \pi} \sigma} \int_{\frac{-T}{2}}^{\frac{T}{2}} \exp \frac{-t^{2}}{2 \sigma^{2}} \cos \left(\frac{2 \pi n}{T} t\right) d t
\end{aligned}
$$

where the term in parenthesis is equal to $n \omega_{0} \mathrm{t}$. Continuing

$$
a_{n}=\frac{N e \omega_{0}}{2 \sigma \pi \sqrt{2 \pi}} \operatorname{Re} \int_{\frac{-T}{2}}^{\frac{T}{2}} \exp \frac{-t^{2}}{2 \sigma^{2}} \exp i n \omega_{0} t d t
$$




$$
=\frac{N e \omega_{0}}{\sigma \pi \sqrt{2 \pi}} \exp -\frac{n^{2} \omega_{0}^{2} \sigma^{2}}{2} \operatorname{Re} \int_{0}^{\frac{T}{2}} \exp \left(-\frac{t}{\sqrt{2} \sigma}-\frac{i n \omega_{0} \sigma}{\sqrt{2}}\right)^{2} d t
$$

If $\frac{T}{2}>>\sigma$, the integral is nearly equal to $\sqrt{2 \pi} \sigma$. [25] and we have

$$
a_{n}=\frac{N e \omega_{0}}{\pi} \exp -\frac{n^{2} \omega_{0}^{2} \sigma^{2}}{2}
$$

The coefficients have the amplitudes as illustrated in Figure 2.2. They are normalized to the $n=0$ harmonic number of the $\sigma=10 \mathrm{ps}$ pulse. The figure demonstrates two important phenomena. First, a Gaussian in the time domain is a Gaussian in the frequency domain. Secondly, as the pulse narrows in the time domain, the broader it becomes in the frequency domain.

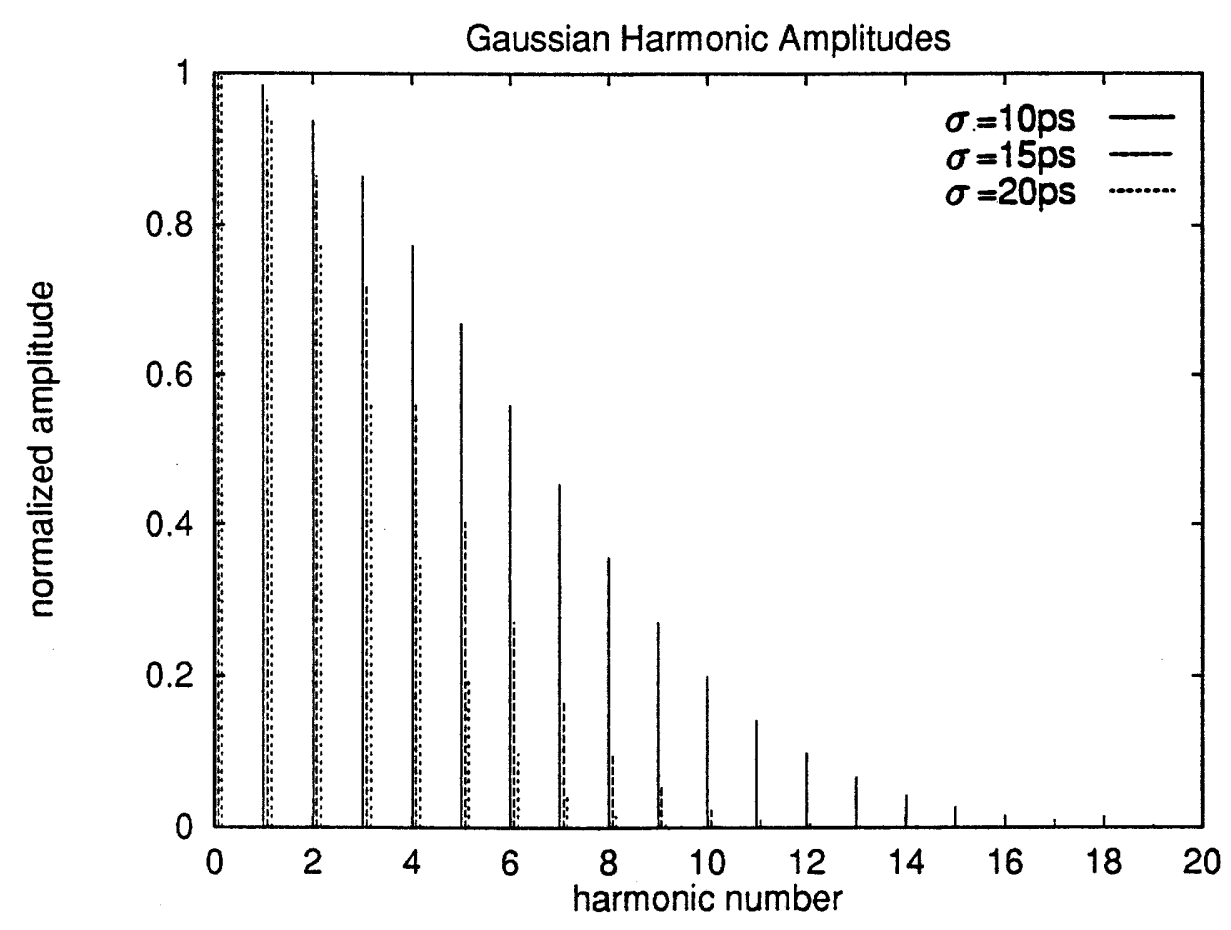

Figure 2.2: Fourier Harmonic Magnitudes

The Fourier series of Equation 2.3 can be written in terms of the beam current $I_{b}$ :

$$
I_{b}(t)=\left\langle I_{b}\right\rangle+2\left\langle I_{b}\right\rangle \sum_{n=1}^{\infty} A_{n} \cos \left(n \omega_{0} t\right)
$$


where $\left\langle I_{b}\right\rangle$ is the total charge divided by the bunching period and $A_{n}$ is the exponential dependence of $a_{n}$.

\subsubsection{Fourier Analysis of a Differentiated Gaussian}

It will be shown that the button pickup used will differentiate the input signal. The Fourier components for a Gaussian pulse that has been differentiated will now be calculated.

A differentiated Gaussian pulse is given by:

$$
\frac{d}{d t} \exp \frac{-t^{2}}{2 \sigma^{2}}=\frac{-t}{\sigma^{2}} \exp \frac{-t^{2}}{2 \sigma^{2}}
$$

and by inspection can be seen that it is an odd function. This has the consequence that only the $b_{n}$ survive from Fourier series of Equation 2.3. Then:

$$
b_{n}=\frac{-N e}{T \sqrt{2 \pi} \sigma^{3}} \int_{\frac{-T}{2}}^{\frac{T}{2}} t \exp \frac{-t^{2}}{2 \sigma^{2}} \sin \left(\frac{2 \pi n}{T} t\right) d t
$$

and:

$$
\begin{aligned}
b_{n} & =\frac{N e}{2 \pi \sigma^{3} \sqrt{2 \pi}} \frac{d}{d n}\left[\int_{\frac{-T}{2}}^{\frac{T}{2}} \exp \frac{-t^{2}}{2 \sigma^{2}} \cos \left(\frac{2 \pi n}{T} t\right) d t\right] \\
& =\frac{N e}{2 \pi \sigma^{3} \sqrt{2 \pi}} \frac{d}{d n}\left[\operatorname{Re} \int_{\frac{-T}{2}}^{\frac{T}{2}} \exp \frac{-t^{2}}{2 \sigma^{2}} \exp \left(i n \omega_{0} t\right) d t\right] \\
& =\frac{N e}{\pi \sigma^{3} \sqrt{2 \pi}} \frac{d}{d n}\left[\exp \left(-\frac{n^{2} \omega_{0}^{2} \sigma^{2}}{2}\right) \operatorname{Re} \int_{0}^{\frac{T}{2}} \exp \left(-\frac{t}{\sqrt{2} \sigma}-\frac{i n \omega_{0} \sigma}{\sqrt{2}}\right)^{2} d t\right]
\end{aligned}
$$

again, if $\frac{T}{2} \gg>$ the integral is nearly equal to $\sqrt{2 \pi} \sigma$. Therefore, the magnitude is

$$
b_{n}=\frac{N e n \omega_{0}^{2}}{\pi} \exp \left(-\frac{n^{2} \omega_{0}^{2} \sigma^{2}}{2}\right)
$$

This result could also have been found by differentiating Equation 2.7 directly. These coefficients have the amplitudes as illustrated in the Figure 2.3 and are nor- 
malized to the $6^{\text {th }}$ harmonic of the $\sigma=10 \mathrm{ps}$ curve. The ratio is seen to peak in the vicinity of the $5^{\text {th }}$ harmonic but drops in magnitude for greater pulse widths.

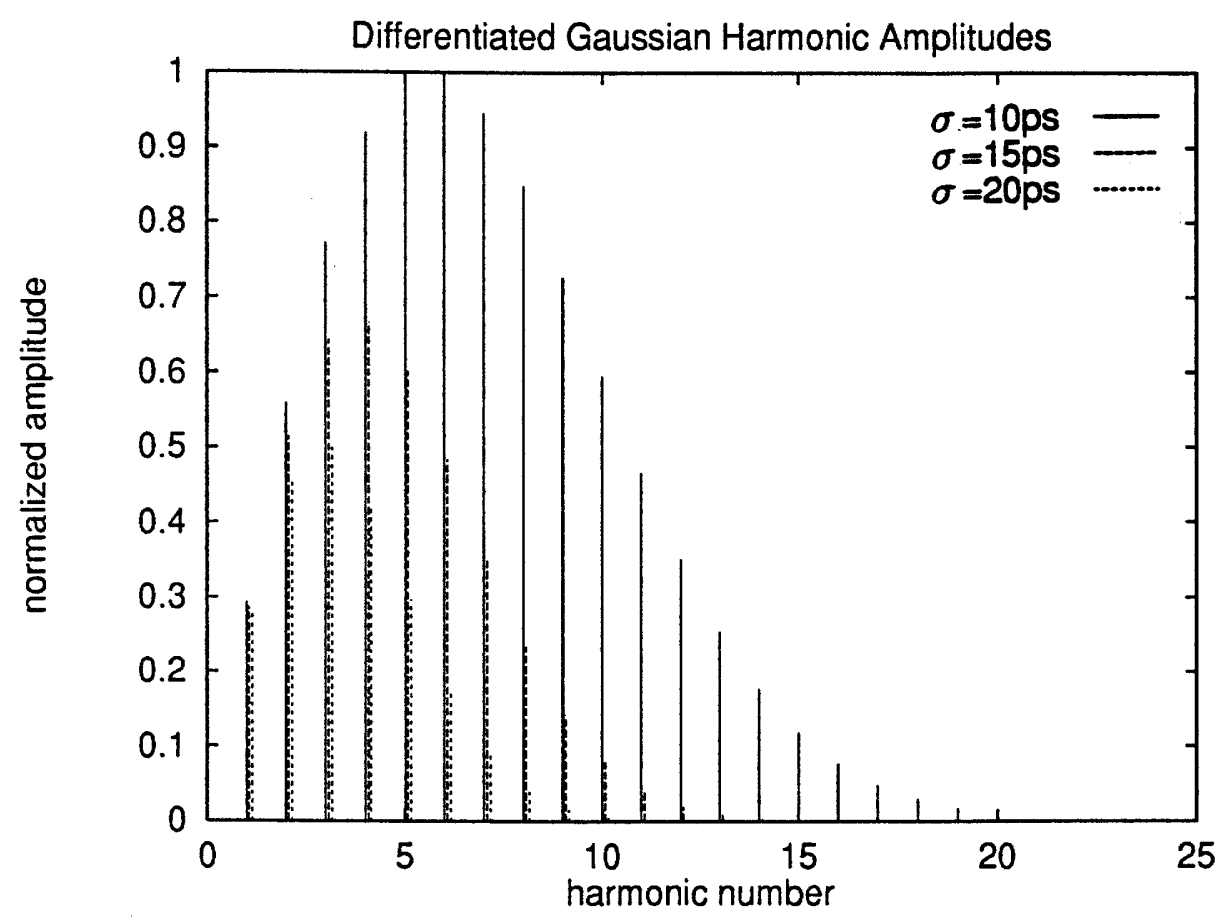

Figure 2.3: Fourier Harmonic Amplitudes for Differentiated Gaussian

\subsection{Theoretical Output}

In the analysis of the physical parameters of the monitor assembly, it is desirable to obtain the equivalent circuit for the button and theoretical values of button impedance and capacitance. By utilizing the previous section, equations for expected button voltage for a given beam input will be shown.

\subsubsection{Equivalent Circuit}

A cut away view of the button position relative to the beam and pipe wall is sketched in Figure 2.4. The capacitance $\mathrm{C}$ is the natural capacitance formed by two conductors with a potential difference. 

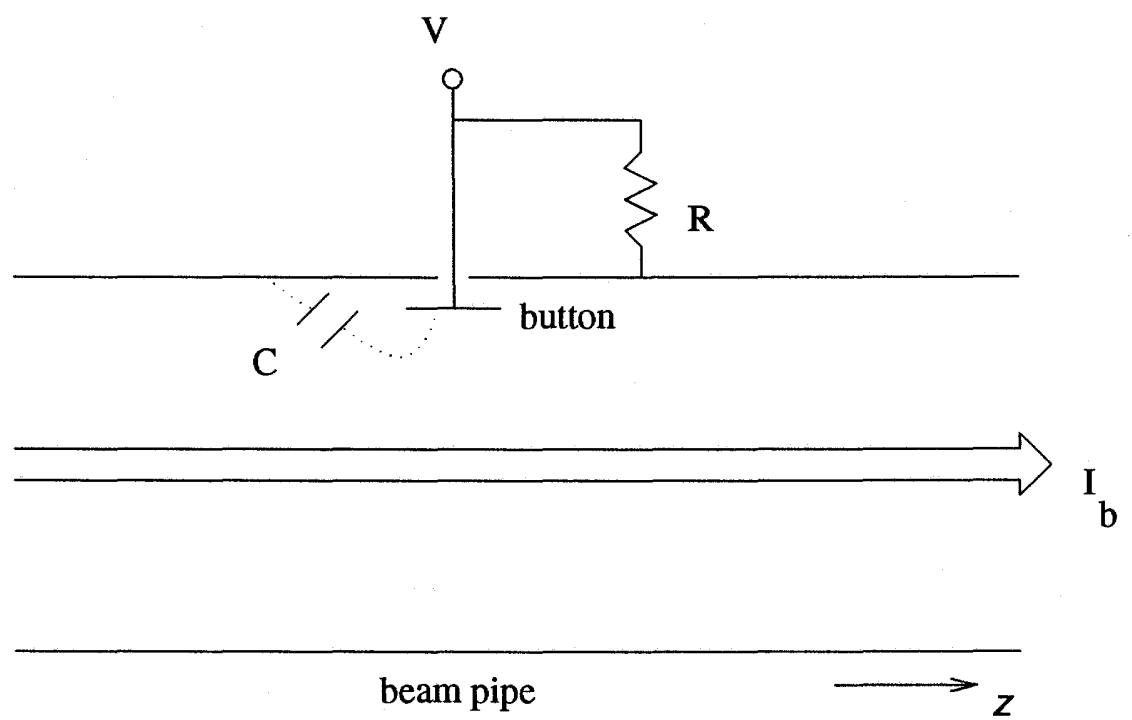

Figure 2.4: Button Orientation

The circuit model for the monitor is obtained by inspection of Figure 2.4 and is illustrated in Figure 2.5.

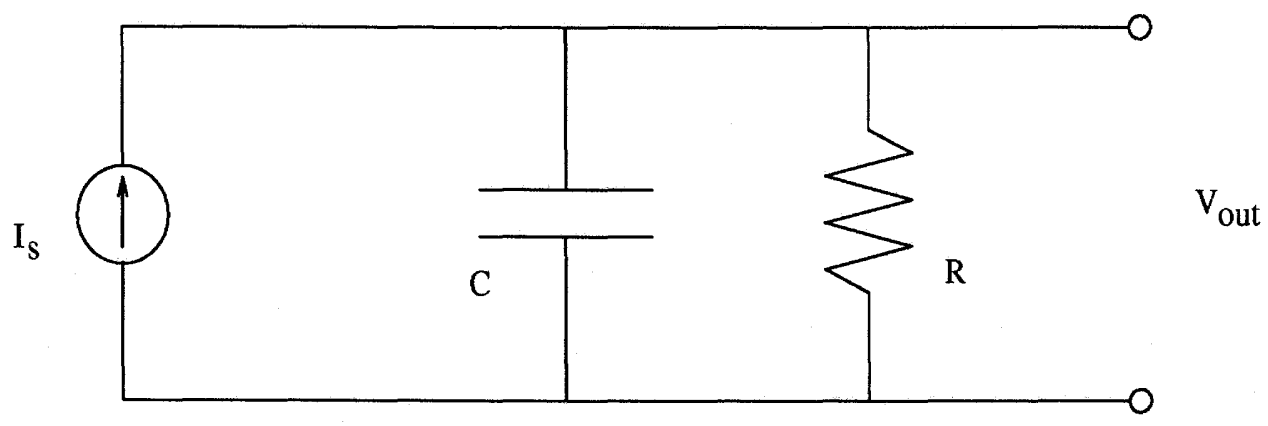

Figure 2.5: Circuit Model

The current source is the button, $\mathrm{C}$ is the capacitance from button to beam pipe wall and $R$ is the termination resistance of the electrode. The output of the voltage pulse can be developed as follows [25].

The charge density in the beam pipe is given by 


$$
q_{b}(t)=\frac{I_{b}(t)}{\beta_{b} c}
$$

where the variable $\beta_{b}$ is the velocity of the electrons of the beam divided by the speed of light $c$.

The image charge on the inside surface of the pipe is opposite in polarity and equal in magnitude to the beam charge. The charge flowing in the button is:

$$
Q_{s}(t)=-\frac{\phi l I_{b}(t)}{2 \pi \beta_{b} c}
$$

where $\phi$ equals the azimuthal width of detector and $l$ is the length of the button in the $z$ direction. If the button is circular, then $\phi l$ is equal to the button area divided by the pipe radius. The current supplied by the probe is then

$$
I_{s}(t)=\frac{\phi l}{2 \pi \beta_{b} c} \frac{d I_{b}(t)}{d t}
$$

As shown below, the capacitance is very small and almost all the current flows through the terminating resistance $I_{s} \simeq I_{R}$

$$
V_{\text {out }}(t)=R I_{R}(t)
$$

With a Gaussian beam current, the voltage out is:

$$
V_{\text {out }}=-\left\{\frac{N e \phi l R}{(2 \pi)^{\frac{3}{2}} \beta_{b} c}\right\} \frac{t}{\sigma^{3}} e^{-\frac{t^{2}}{2 \sigma^{2}}}
$$

which is a Gaussian doublet resembling Figure 2.6. The figure was obtained from Equation 2.12 with the variables in the curly brackets lumped together as a unitless constant equal to one and $\sigma=8$ ps.

The peak output voltage will occur at approximately $t= \pm \sigma$ and from Equation 2.12 equals 


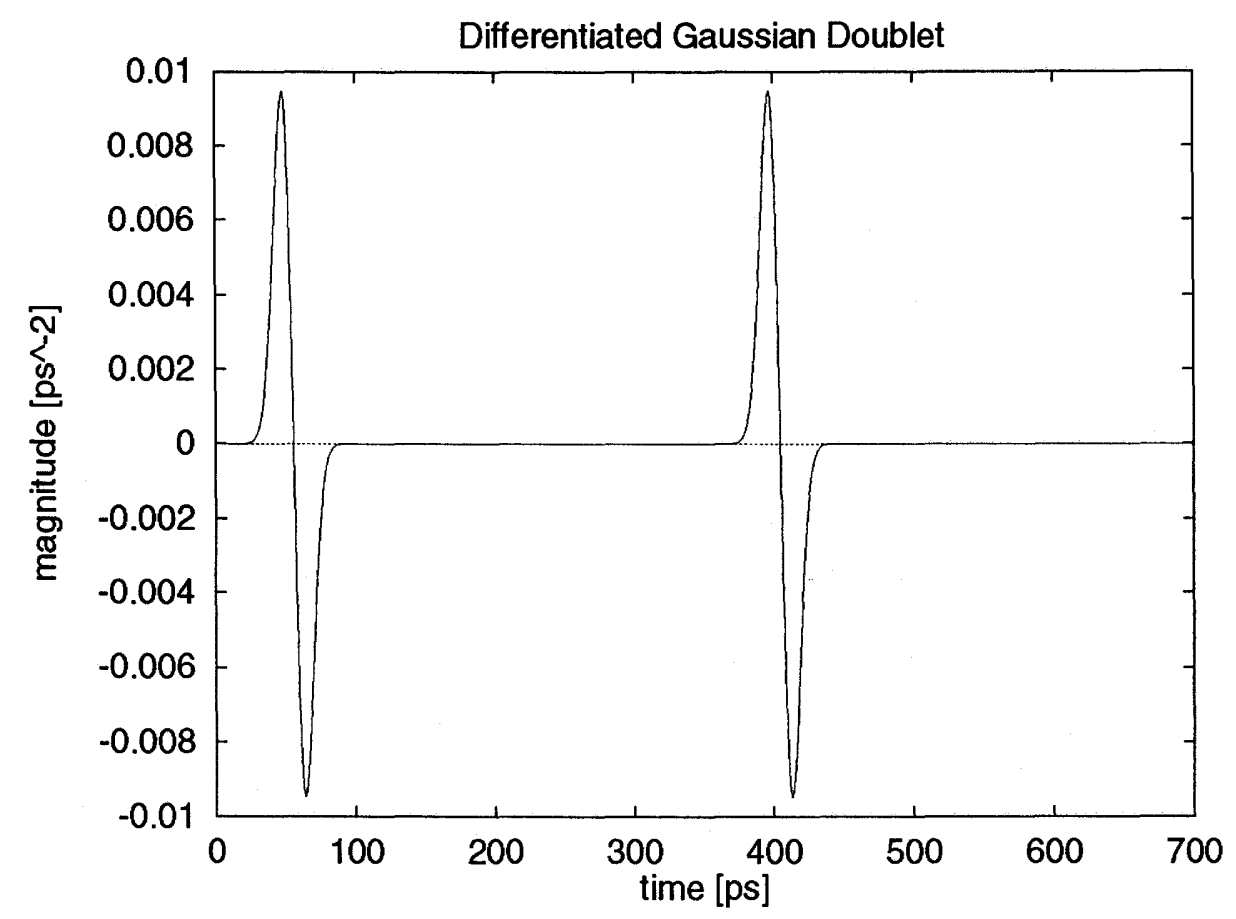

Figure 2.6: Differentiated Gaussian Wavepackets

$$
V_{\text {peak }}=\frac{N e \phi l R}{(2 \pi)^{\frac{3}{2}} \beta_{b} c} \frac{e^{-1 / 2}}{\sigma^{2}}
$$

The rms voltage output for each harmonic of the input wave can be found by using Equations 2.7 and 2.11. This is found to be for a circular button:

$$
V_{\text {out }}(\omega)=R I_{s}=\frac{R N e r^{2} n \omega_{0}}{\sqrt{2} T_{b} \rho \beta_{b} c} A_{n}
$$

where $r$ is the radius of the circular button and $\rho$ is the radius of the beam pipe. $N e$ can be found by integrating the bunch current $I_{b}$ over its width in time

$$
\int I_{b} d \tau=N e
$$




\subsubsection{Button Impedance}

To get an idea of the magnitude of actual impedance seen by the beam due to the button is, the actual impedance of the capacitor and resistor in parallel is

$$
Z(\omega)=\frac{R}{1+j \omega R C}
$$

With the substitution of $Z(\omega)$ for $R$ in the previous equations, and the voltage of Equation 2.14 is divided by the current from Equation 2.7, the impedance for a circular button is:

$$
Z_{b}(\omega)=\frac{V_{o u t}(\omega)}{I_{b}(\omega)}=\frac{\omega r^{2}}{2 \rho \beta_{b} c} \frac{R}{1+j \omega R C}
$$

The above result agrees with Goldberg [11]. The value of $R$ is the characteristic impedance of the coaxial cable and $C$ can be obtained from the equation for parallel plate capacitors. It is desirable for the capacitance to be very small, since this provides a time constant which is small compared to the charge collection time. This small time constant will provide good timing information since $R C \ll \mathrm{t}_{c} \quad[16]$. 


\section{Chapter 3}

\section{Filter Simulations}

Simulations provide an important tool in the verification of developed theory. This chapter contains a digital simulation of the output of the monitor assembly. Filters are located at the button pickoff and are needed to select two sufficiently different frequency components of the beam; one at the first harmonic of the machine frequency and one at the fourth. For an input waveform, the filters need to be fast enough in the time domain to respond to short bunching and yet narrow enough in the frequency domain to limit the feedthrough of unwanted harmonics. For each filter, the transfer function is found and the results of a computer simulation are shown.

\subsection{Transfer Function}

The required bandpass (BP) filters were obtained from Rf Microwave Ltd. [20]. They are resonant cavity two pole filters with the specifications listed in Table 3.1.

Table 3.1: Filter Specifications

\begin{tabular}{|c|c|c|c|c|}
\hline $\mathrm{f}_{0} \mathrm{MHz}$ & $3 \mathrm{~dB} \% \mathrm{f}_{0}$ & $3 \mathrm{~dB} \mathrm{MHz}$ & $20 \mathrm{~dB} \% \mathrm{f}_{0}$ & $20 \mathrm{~dB} \mathrm{MHz}$ \\
\hline 2856 & 0.5 & 14 & 1.5 & 43 \\
\hline 11424 & 0.5 & 57 & 1.5 & 171 \\
\hline
\end{tabular}

The tables used by Geis [10] define the width of the passband as the frequencies at which the maximum variation is equal to the passband variation. Since Chebychev filters are used, there is a ripple in the passband that will define the passband width. From the filter specifications, the $3 \mathrm{~dB}$ point occurs at $0.5 \%$ of the center frequency. It is assumed that the passband width as defined by Geis is $0.35 \%$ of the center frequency. A sketch of the desired frequency response is illustrated in Figure 3.1, and 
Table 3.2 containing the resultant numbers.

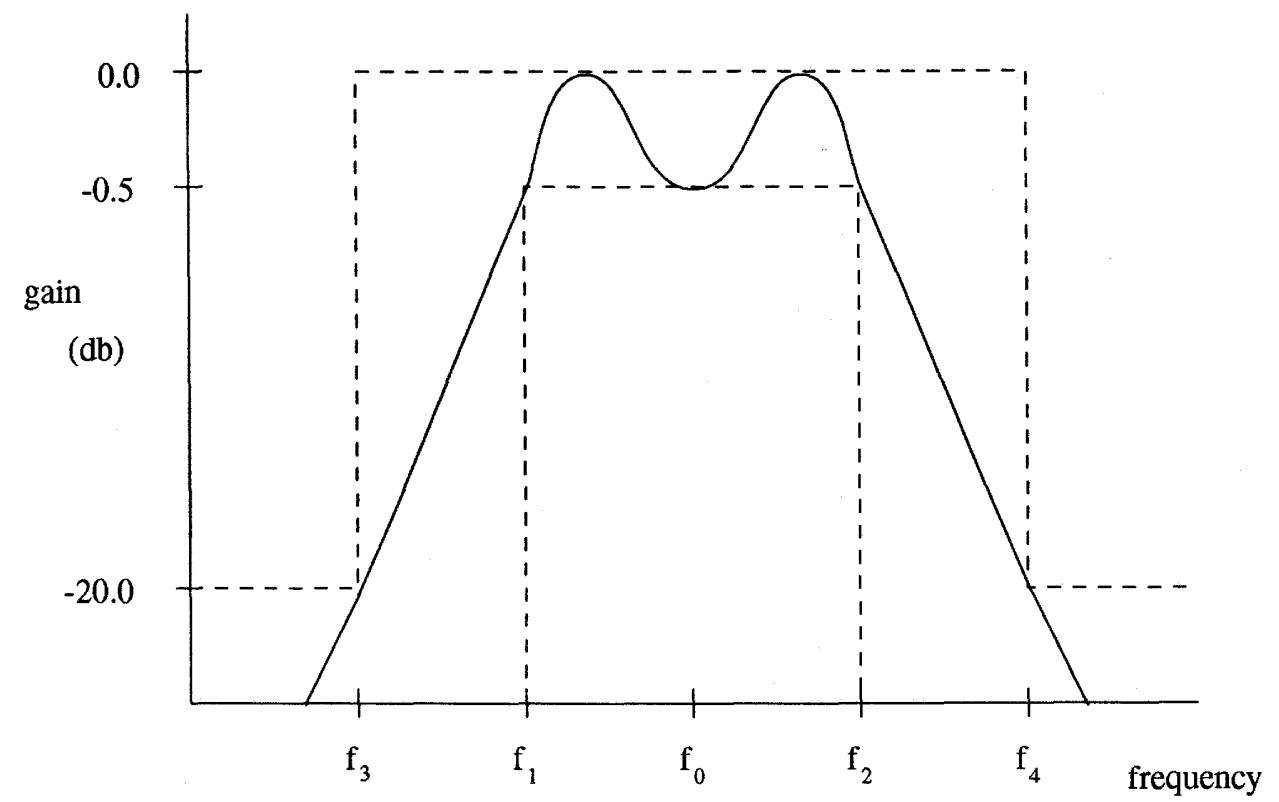

Figure 3.1: Desired Frequency Response

Table 3.2: Frequency Numbers

\begin{tabular}{|c|c|c|}
\hline symbol & \multicolumn{2}{|c|}{ frequency $\mathrm{MHz}$} \\
\hline $\mathrm{f}_{0}$ & 2856 & 11424 \\
\hline $\mathrm{f}_{1}$ & 2846 & 11384 \\
\hline $\mathrm{f}_{2}$ & 2866 & 11464 \\
\hline $\mathrm{f}_{3}$ & 2813 & 11253 \\
\hline $\mathrm{f}_{4}$ & 2899 & 11595 \\
\hline
\end{tabular}

With the numbers from Table 3.2 and the nomographs supplied by Geis [10], a filter of order two will have the desired response at both harmonics. This confirms the specifications from RF Microwave Ltd. [20].

The circuit for the lowpass (LP) prototype is illustrated in Figure 3.2, which is then transformed with the appropriate substitutions to the BP filter circuit of Figure 3.3 . 


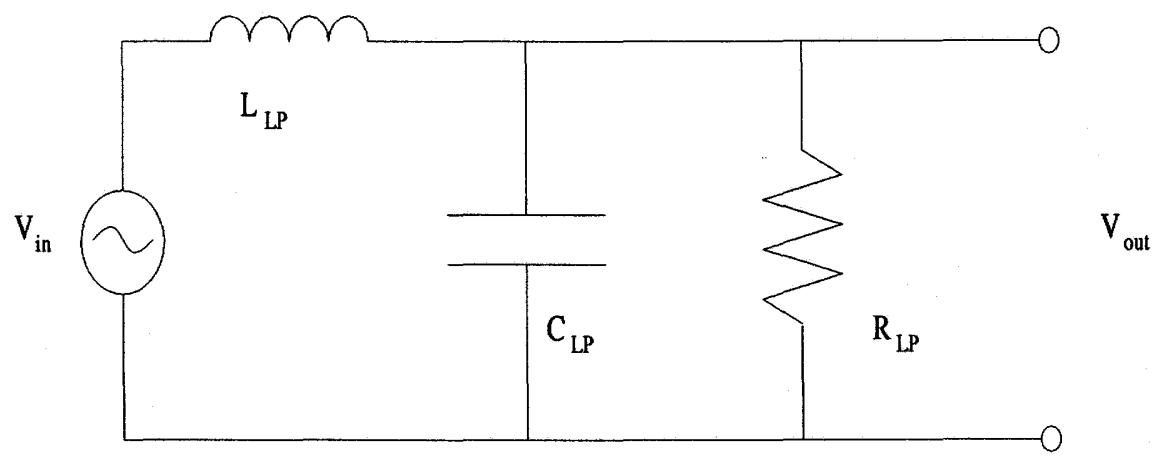

Figure 3.2: Low Pass Filter

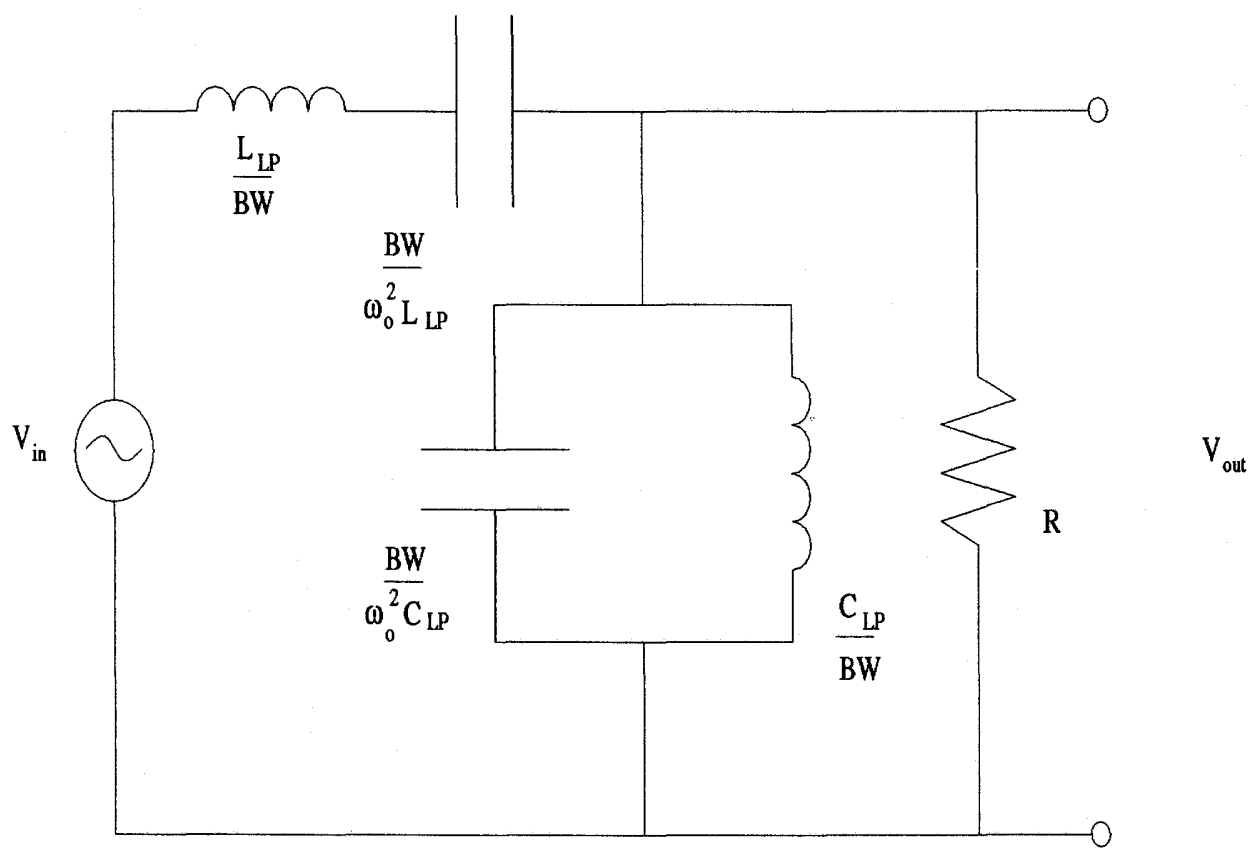

Figure 3.3: Bandpass Filter

To obtain the transfer function of the BP circuit, the voltage divider principle is applied in the Laplace domain

$$
\begin{gathered}
z_{1}=\left(s L^{\prime}+\frac{1}{s C^{\prime}}\right) \\
z_{2}=\left(\frac{1}{s L}+s C+\frac{1}{R}\right)^{-1}
\end{gathered}
$$




$$
\frac{V_{\text {out }}}{V_{\text {in }}}=\frac{z_{2}}{z_{1}+z_{2}}
$$

where $L^{\prime}$ and $C^{\prime}$ are the series inductance and capacitance respectively and $L$ and $C$ are the parallel inductance and capacitance respectively. After simplifying

$$
\frac{V_{\text {out }}}{V_{\text {in }}}=\frac{L C^{\prime} R s^{2}}{L^{\prime} C^{\prime} L C R s^{4}+L^{\prime} C^{\prime} L s^{3}+\left(C L+L^{\prime} C^{\prime}+C^{\prime} L\right) R s^{2}+L s+R}
$$

The corresponding numbers for the ideal BP filter are illustrated in Table 3.3.

Table 3.3: Component Values

\begin{tabular}{|c|c|c|}
\hline symbol & $2856 \mathrm{MHz}$ & $11424 \mathrm{MHz}$ \\
\hline$L$ & $27.807 \mathrm{pH}$ & $6.9547 \mathrm{pH}$ \\
\hline$C$ & $111.587 \mathrm{pF}$ & $27.9078 \mathrm{pF}$ \\
\hline$L^{\prime}$ & $373983.8 \mathrm{pH}$ & $93533.37 \mathrm{pH}$ \\
\hline$C^{\prime}$ & $0.0082970 \mathrm{pF}$ & $0.0020751 \mathrm{pF}$ \\
\hline$R$ & $50 \Omega$ & $50 \Omega$ \\
\hline
\end{tabular}

If the simulation is scaled so that the $1^{s t}$ harmonic period is 350 and the $4^{\text {th }}$ harmonic is $\frac{350}{4}$, the scale has moved from picoseconds to seconds. The transfer function after frequency scaling is then for the $1^{\text {st }}$ harmonic:

$$
\frac{V_{\text {out }}}{V_{\text {in }}}=\frac{11.536 s^{2}}{481406138 s^{4}+86283 s^{3}+310304 s^{2}+27.81 s+50}
$$

and for the $4^{\text {th }}$

$$
\frac{V_{\text {out }}}{V_{\text {in }}}=\frac{0.72179 s^{2}}{1883561 s^{4}+1349.9 s^{3}+19409.8 s^{2}+6.9547 s+50}
$$

Substituting $s=j \omega$ into Equation 3.2 and rewriting in phasor form, the Bode plot is obtained in Figure 3.4. This is also done for the fourth harmonic of Equation 3.3 and illustrated in Figure 3.5. 


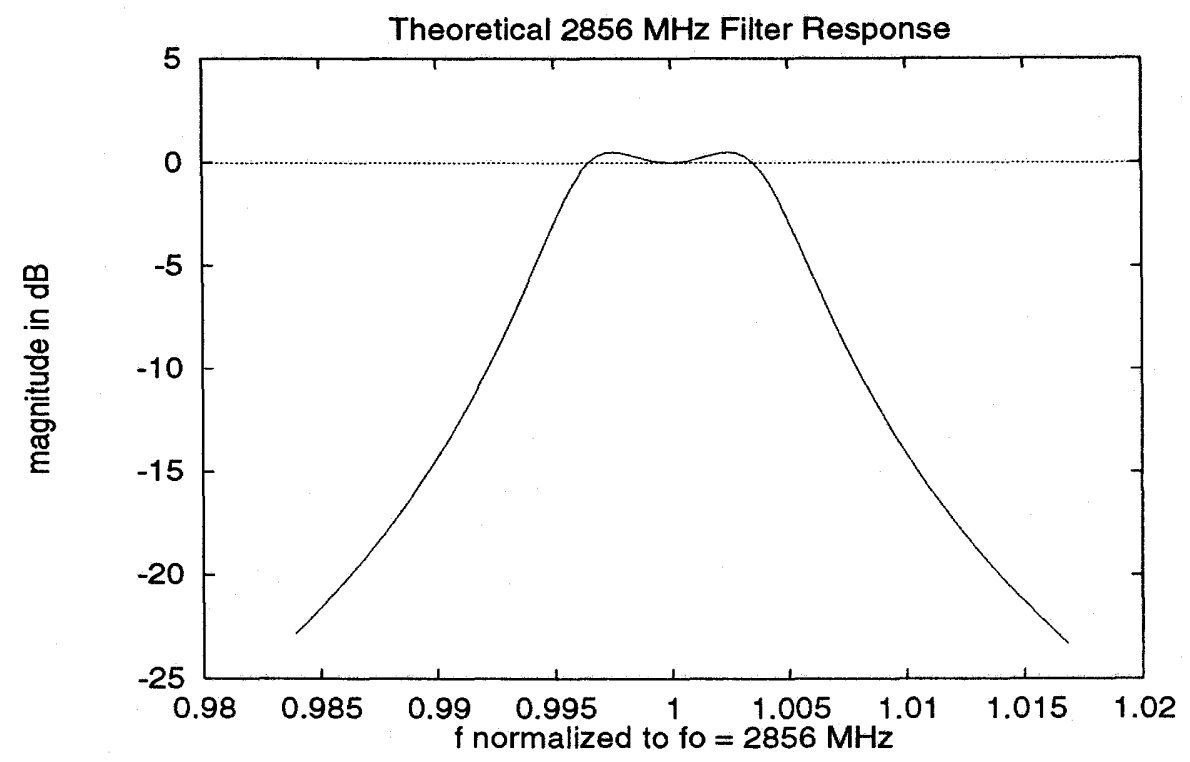

Figure 3.4: Bandpass Filter Response for First Harmonic

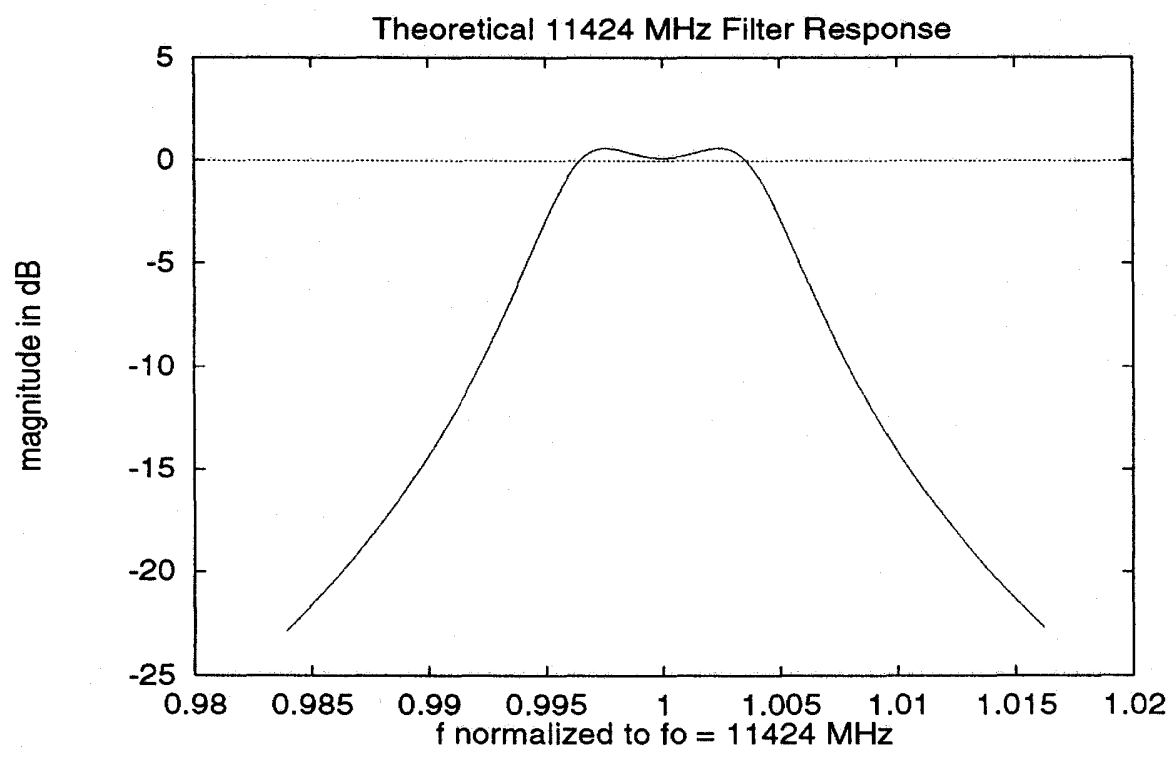

Figure 3.5: Bandpass Filter Response for Fourth Harmonic

\subsection{Digital Simulation}

To simulate the response of the monitor circuit and filters, a digital computer and time-sampling method was used. The time-sampling method involves obtaining the 
Z-transform from the analogue transfer function of the filters, then transforming it to a difference equation from which the time response can be obtained for an arbitrary input using a computer program.

The Z-transforms for Equations 3.2 and 3.3 were obtained from a computer program called Octave. The Laplace transfer function is typed in, the sampling period is selected and the digital transfer function is the output. The sampling period is determined from the poles of the transfer function. For a quadratic denominator in an analog transfer function, the progression from the Laplace domain to the $\mathrm{Z}$ domain is

$$
(s+a)^{2}+b^{2} \rightarrow z^{2}-2 z e^{-a T} \cos b T+e^{-2 a T} \rightarrow\left(z-z_{1}\right)\left(z-\overline{z_{1}}\right)
$$

where $z_{1}=e^{-a T} e^{j b T}=e^{-a T} \angle b T$. For the poles, the real component is $a$ and the complex component is $b$.

In order for the sampling period to have an accurate effect [23], many samples should occur in the period of the time constant which is defined as $\frac{1}{a}$. Also, for a complex pole many samples per period of the sinusoid should be done or $b T<1$.

The poles of Equations 3.2 and 3.3 are located at $-4.47 \mathrm{E}-5 \pm \mathrm{i} 1.8008 \mathrm{E}-2$ and $4.47 \mathrm{E}-5 \pm \mathrm{i} 1.7882 \mathrm{E}-2$ for the $1^{\text {st }}$ harmonic and $-1.80 \mathrm{E}-4 \pm \mathrm{i} 7.2032 \mathrm{E}-2$ and $-1.80 \mathrm{E}-4$ \pm i7.1527E-2 for the $4^{\text {th }}$ harmonic.

Careful consideration must be given on the selection of the sampling period. If it is too small, the computer rounding errors will cause the program not to converge. If the period is too big, the output will lose accuracy. The various sampling periods used are displayed in Table 3.4 along with the corresponding coefficients.

For reference, Octave displays [9] polynomials in the format

$$
Y(z)=\frac{a_{3} z^{3}+a_{2} z^{2}+a_{1} z^{1}+a_{0}}{z^{4}+b_{3} z^{3}+b_{2} z^{2}+b_{1} z^{1}+b_{0}} X(z) .
$$

The difference equation that was used in the computer program was obtained from the $\mathrm{Z}$ domain transfer function Equation 3.4. After multiplying top and bottom by 
Table 3.4: Coefficients for Discrete Equation

\begin{tabular}{|c|c|c|c|c|}
\hline coefficient & \multicolumn{2}{|c|}{$2856 \mathrm{MHz}$} & \multicolumn{2}{c|}{$11424 \mathrm{MHz}$} \\
\hline & $(T=0.5 \mathrm{ps})$ & $(T=1.0 \mathrm{ps})$ & $(T=0.1 \mathrm{ps})$ & $(T=1.0 \mathrm{ps})$ \\
\hline$a_{3}$ & $2.995204529 \mathrm{E}-9$ & $1.197997123 \mathrm{E}-8$ & $1.916948822 \mathrm{E}-9$ & $1.914908103 \mathrm{E}-7$ \\
\hline$a_{2}$ & $-2.9952973435 \mathrm{E}-9$ & $-1.198068932 \mathrm{E}-8$ & $-1.916990122 \mathrm{E}-9$ & $-1.915365697 \mathrm{E}-7$ \\
\hline$a_{1}$ & $-2.9950188996 \mathrm{E}-9$ & $-1.197853727 \mathrm{E}-8$ & $-1.916865333 \mathrm{E}-9$ & $-1.913992924 \mathrm{E}-7$ \\
\hline$a_{0}$ & $2.995111825 \mathrm{E}-9$ & $1.197925458 \mathrm{E}-8$ & $1.916906966 \mathrm{E}-9$ & $1.914450511 \mathrm{E}-7$ \\
\hline$b_{3}$ & -3.999749250 & -3.999176260 & -3.999825181 & -3.988976491 \\
\hline$b_{2}$ & 5.999408910 & 5.998173464 & 5.999578677 & 5.977266560 \\
\hline$b_{1}$ & -3.999570032 & -3.998817884 & -3.999681803 & -3.987546835 \\
\hline$b_{0}$ & 0.9999103875 & 0.9998207831 & 0.9999283094 & 0.9992833251 \\
\hline
\end{tabular}

$z^{-4}$ the equation becomes:

$$
Y(z)=\frac{a_{3} z^{-1}+a_{2} z^{-2}+a_{1} z^{-3}+a_{0} z^{-4}}{1+b_{3} z^{-1}+b_{2} z^{-2}+b_{1} z^{-3}+b_{0} z^{-4}} X(z)
$$

and knowing that:

$$
X(z) z^{-n} \rightarrow x[(k-n) T]
$$

where $n$ becomes a delay in time, the discrete equation becomes

$$
\begin{gathered}
y(k T)=a_{3} x[(k-1) T]+a_{2} x[(k-2) T]+a_{1} x[(k-3) T]+a_{0} x[(k-1) T] \\
-b_{3} y[(k-1) T]-b_{2} y[(k-2) T]-b_{1} y[(k-3) T]-b_{0} y[(k-4) T]
\end{gathered}
$$

Since the system is causal, $y(-q)$ equals zero when $q$ is any positive real number.

With a sinusoidal input of amplitude 1 and sampling periods of $T=0.5 \mathrm{ps}$ for the $1^{\text {st }}$ harmonic and $T=0.1 \mathrm{ps}$ for the $4^{\text {th }}$, the simulations for the output of the filters are plotted in Figures 3.6 and 3.7. 


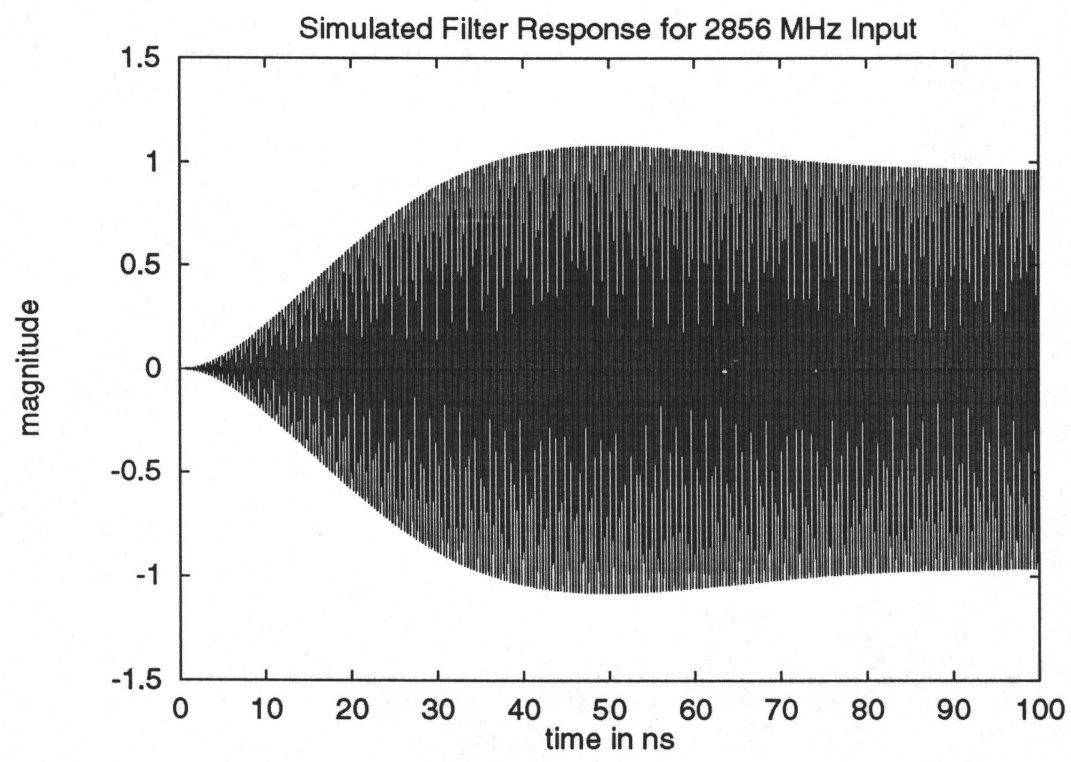

Figure 3.6: First Harmonic Response

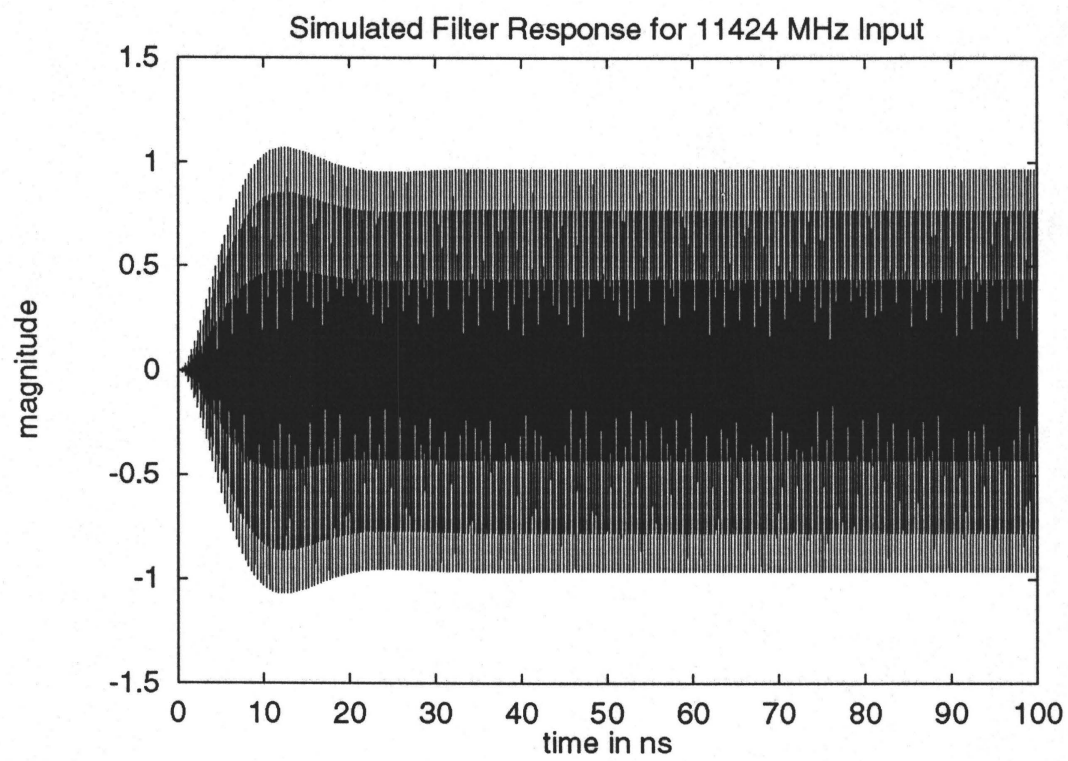

Figure 3.7: Fourth Harmonic Response 
A closeup of the waveform of Figure 3.6 would illustrate a period of $350 \mathrm{ps}$ and 87.5 ps for Figure 3.7. It is clearly visible that the filters reach steady state at 100 ns for the $2856 \mathrm{MHz}$ case and $25 \mathrm{~ns}$ for the $11424 \mathrm{MHz}$ case. This is within 5 time constants for each filter. A summary of values obtained from theory, bench test and digital simulations are illustrated in Table 3.5. The theory numbers are obtained from Figures 3.4 and 3.5. The bench test numbers were obtained by selecting an input to the filters of known magnitude and measuring the output as described in Chapter 4. The simulation numbers were obtained from Figures 3.6 and 3.7 at $\approx 100 \mathrm{~ns}$.

Table 3.5: Theoretical, Simulated and Experimental Values

\begin{tabular}{|c|c|c|c|}
\hline \multirow{2}{*}{$\begin{array}{c}\text { input } \\
\text { frequency } \mathrm{MHz}\end{array}$} & \multicolumn{3}{|c|}{ Attenuation dB } \\
\cline { 2 - 4 } & theory & simulation & experimental \\
\hline 2856 & -0.00 & -0.11 & 0.00 \\
\hline 2840 & -4.41 & -4.33 & -3.26 \\
\hline 2830 & -12.62 & -12.53 & -15.89 \\
\hline 2815 & -20.80 & -20.89 & -29.64 \\
\hline & & & \\
\hline 11424 & 0.12 & -0.09 & -0.09 \\
\hline 11360 & -4.45 & -4.41 & -2.95 \\
\hline 11320 & -12.64 & -12.62 & -15.60 \\
\hline 11240 & -22.86 & -22.86 & -28.29 \\
\hline
\end{tabular}

The experimental numbers exhibit somewhat faster edges, but in general the three sets of numbers agree. For the simulations of a differentiated Gaussian input, Equation 2.12 with the braces normalized out and $\sigma=8.0$ ps was used. The Figures 3.8 and 3.9 are the output. 


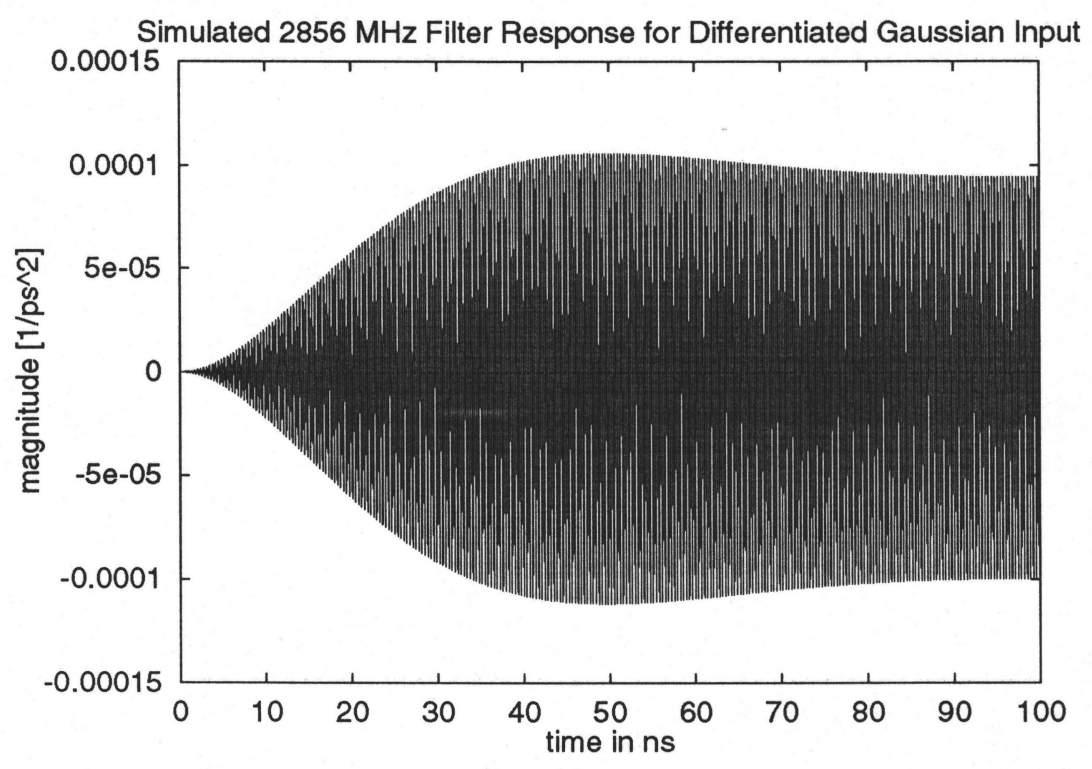

Figure 3.8: First Harmonic Filter Response for $\sigma=8.0 \mathrm{ps}$

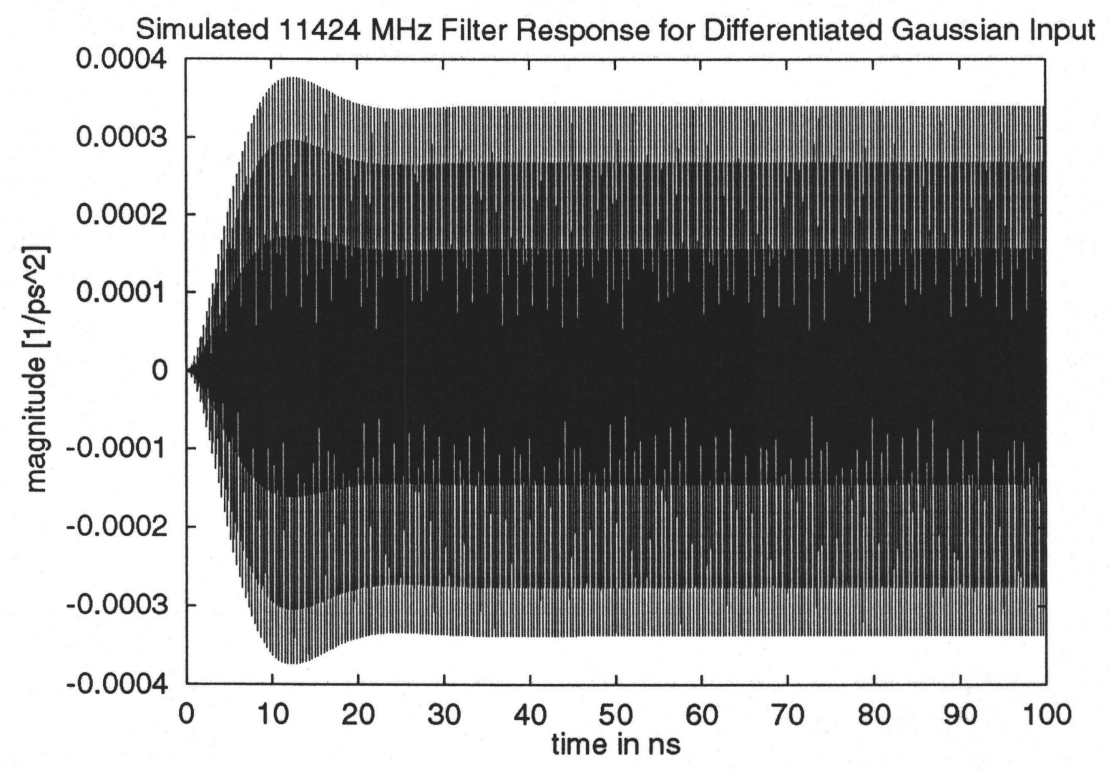

Figure 3.9: Fourth Harmonic Filter Response for $\sigma=8.0 \mathrm{ps}$ 


\subsection{Summary}

Table 3.6 contains the values of several simulation runs, with the maximum values of the output taken at approximately $100 \mathrm{~ns}$, which is the steady-state output. The theoretical values were obtained from Equation 2.10 with the total current $\mathrm{Ne}$ normalized out.

The filter simulation amplitudes agree well with the expected values obtained from Equation 2.10. Given the pulse length of the SAL injector, the reponse time of $100 \mathrm{~ns}$ is fast enough for the filters to be used for the bunch length monitor.

Table 3.6: Output Values for Differentiated Gaussian

\begin{tabular}{|c|c|c|c|c|c|c|}
\hline \multirow{2}{*}{$\sigma$} & \multicolumn{2}{|c|}{$2856 \mathrm{MHz}$} & \multicolumn{2}{|c|}{$11424 \mathrm{MHz}$} & \multirow{2}{*}{} \\
\cline { 2 - 5 } & simulation & theory & simulation & theory & \multirow{2}{*}{$\frac{11424}{2856}$ sim } & \multirow{2}{*}{$\frac{11424}{256}_{\text {theo }}$} \\
\cline { 2 - 5 } & $\mathrm{E}-4\left[1 / \mathrm{ps}^{2}\right]$ & $\mathrm{E}-4\left[1 / \mathrm{ps}^{2}\right]$ & $\mathrm{E}-4\left[1 / \mathrm{ps}^{2}\right]$ & $\mathrm{E}-4\left[1 / \mathrm{ps}^{2}\right]$ & & \\
\hline 8.0 & 1.0036 & 1.0153 & 3.4792 & 3.4792 & 3.4667 & 3.4267 \\
\hline 10.0 & 0.9978 & 1.0094 & 3.1708 & 3.1708 & 3.1780 & 3.1413 \\
\hline 12.0 & 0.9907 & 1.0023 & 2.8307 & 2.8307 & 2.8571 & 2.8242 \\
\hline 14.0 & 0.9825 & 0.9939 & 2.4756 & 2.4756 & 2.5201 & 2.4924 \\
\hline 16.0 & 0.9730 & 0.9844 & 2.1207 & 2.1208 & 2.1795 & 2.1545 \\
\hline 18.0 & 0.9624 & 0.9736 & 1.7797 & 1.7797 & 1.8492 & 1.8284 \\
\hline 20.0 & 0.9507 & 0.9618 & 1.4630 & 1.4630 & 1.5389 & 1.5224 \\
\hline 22.0 & 0.9379 & 0.9489 & 1.1781 & 1.1782 & 1.2560 & 1.2426 \\
\hline
\end{tabular}




\section{Chapter 4}

\section{The SAL Bunch Length Monitor}

The SAL bunch length monitor consists of a button port welded to a $35 \mathrm{~cm}$ length of beam pipe. The stainless steel beam pipe is $6 \mathrm{~cm}$ in diameter with vacuum seal flanges at each end. The button port is fashioned from a vacuum sealed type- $\mathrm{N}$ receptacle and located $8 \mathrm{~cm}$ from one end. The inner conductor of the receptacle protrudes into the interior of the pipe, and is flared to allow interchanging of button studs.

There were six buttons made for the bench tests, three circular and three square. The sizes selected were $0.5,1.0$ and $2.2 \mathrm{~cm}$ in diameter for the circular buttons and $0.5,1.0$ and $2.0 \mathrm{~cm}$ for the lengths of the square sides. All buttons were made of $2 \mathrm{~mm}$ copper with brass stubs inserted into the copper and then soldered. The total distance from the inside surface of the pipe to the inside surface of the button was $3 \mathrm{~mm}$. Figure 4.1 illustrates the monitor as it appeared for the bench tests. The plates on the ends were used to mount type- $\mathrm{N}$ bulkhead receptacles with a thin wire stretched between them. The inside surface of the plates had a $0.15 \mathrm{~mm}$ layer of aluminum glued to them.

\subsection{The Bench Test}

The bench tests were necessary for two reasons. First, to establish that the buttons would provide a signal of significant power to be measurable. Secondly, that a relationship between button shape and output signal could be observed and with this information select the best button for the beam test.

\subsubsection{Component Characterization}

Some preliminary measurements needed to be completed before the actual tests could start. These included measuring the response curves for the filters and diode detectors 


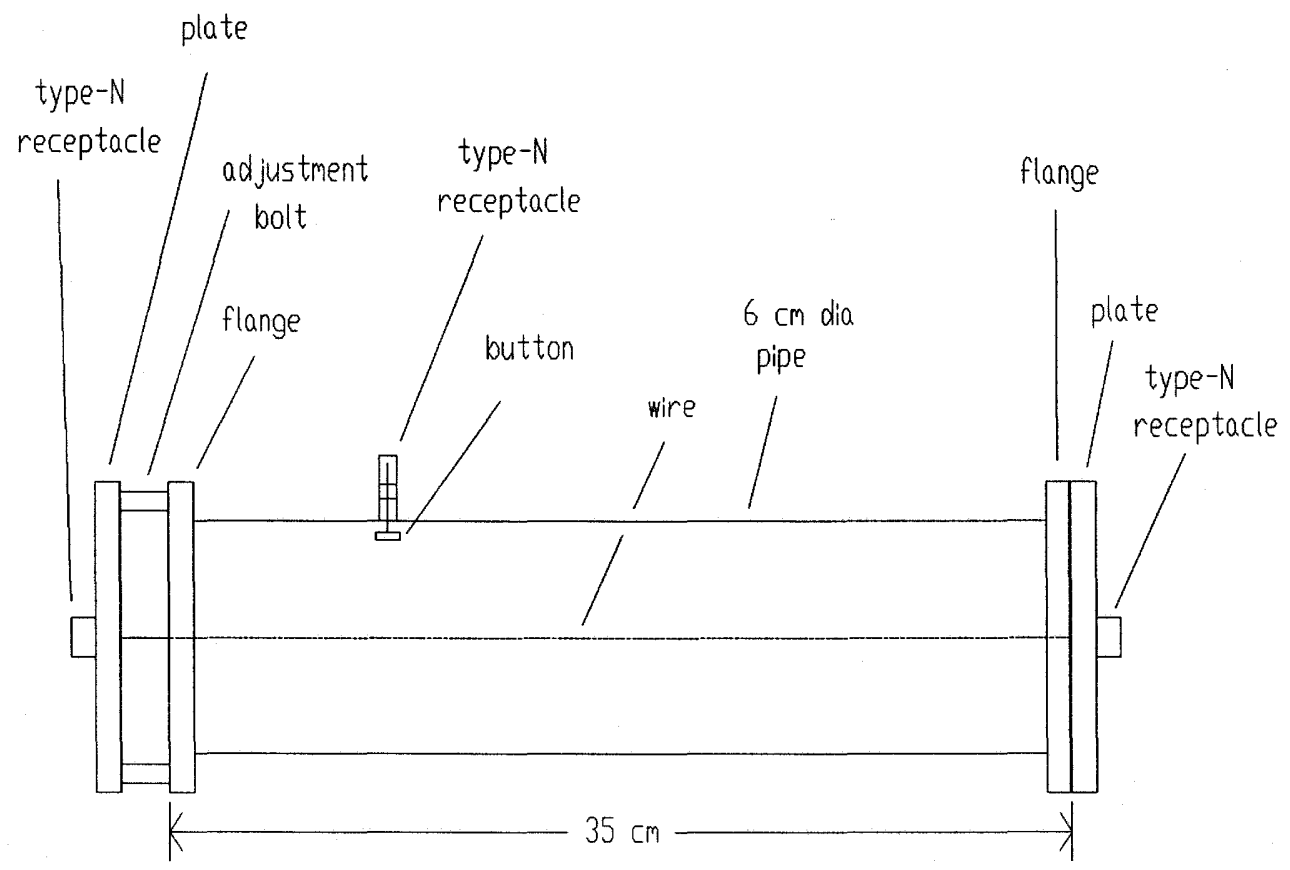

Figure 4.1: Bunch Length Monitor with Test Fixtures

and a measurement of the capacitance of the installed button.

\section{Filter Measurements}

The filters from RF Microwave were measured for their response over the bandwidth concerned. The test setup consisted of the HP 8672A signal generator with an output of $0 \mathrm{dbm}$ set at the center frequency of the $2856 \mathrm{MHz}$ filter. The filter was placed at the signal generator output and a HP 436A Power Meter was referenced to the filter output. The signal frequency was then adjusted at a few $\mathrm{MHz}$ per measurement and the output of the filter as measured by the power meter was recorded. This procedure was also done for the $11424 \mathrm{MHz}$ filter. The results are illustrated in Figures 4.2 and 4.3 , with the results of the theoretical response curves from Chapter 3 included. 


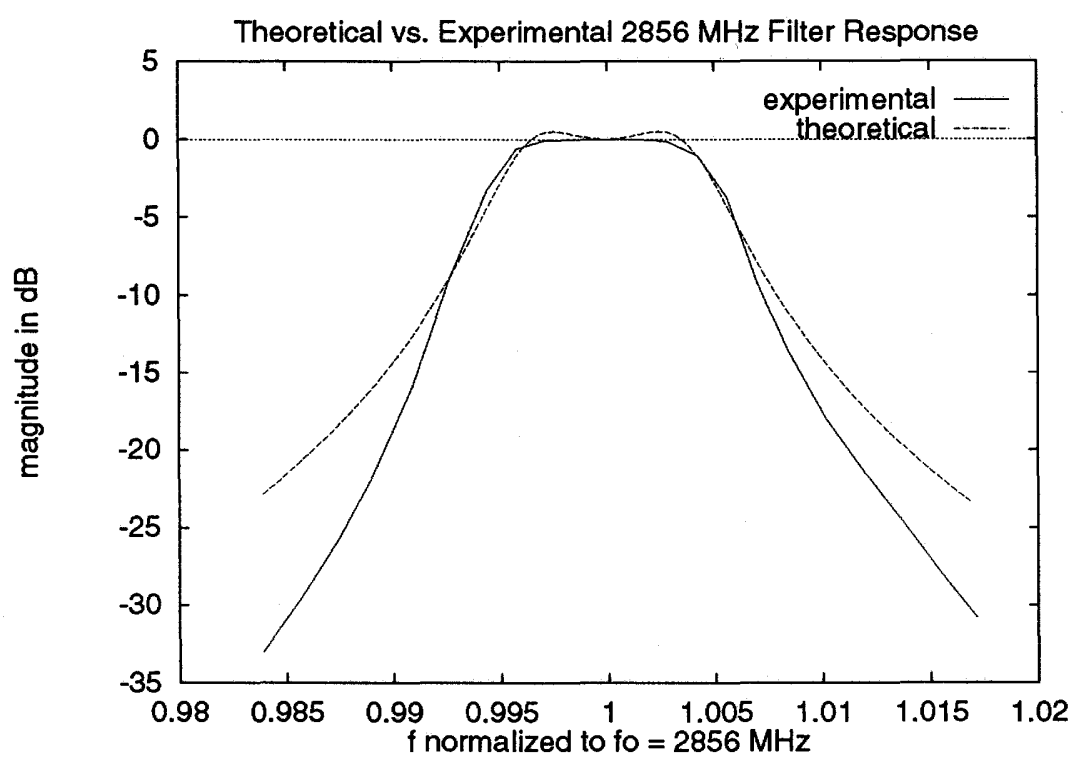

Figure 4.2: Theoretical and Experimental First Harmonic Response

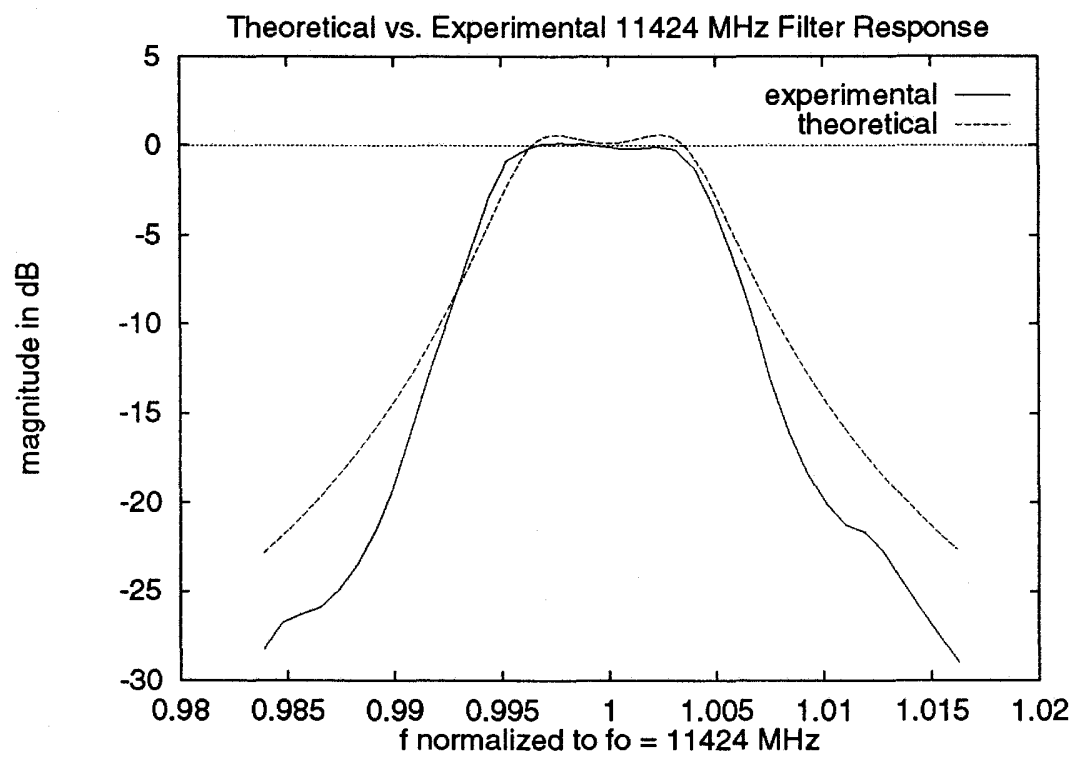

Figure 4.3: Theoretical and Experimental Fourth Harmonic Response 


\section{Detector Measurements}

A similar procedure was used for the crystal detectors. The signal generator was set at the respective center frequency, either $2856 \mathrm{MHz}$ or $11424 \mathrm{MHz}$, with the output power being varied in magnitude. The signal generator output is connected to the input of the crystal detector and the output of the detector is connected to an oscilloscope. A relationship between input power and output voltage can then be found as illustrated in Figures 4.4 and 4.5 .

\section{Capacitance Measurement}

The capacitance measurement was done with a capacitance meter of $0.1 \mathrm{pF}$ sensitivity. One microclip lead was placed on the outer conductor of the button port and the other microclip on the inside conductor. The combined capacitance of the button port and $1 \mathrm{~cm}$ diameter button was measured to be $6.2 \mathrm{pF}$. With the button removed it was measured to be $5.9 \mathrm{pF}$. Since the capacitances of the receptacle and button are in parallel, the button capacitance is $0.3 \mathrm{pF}$. 


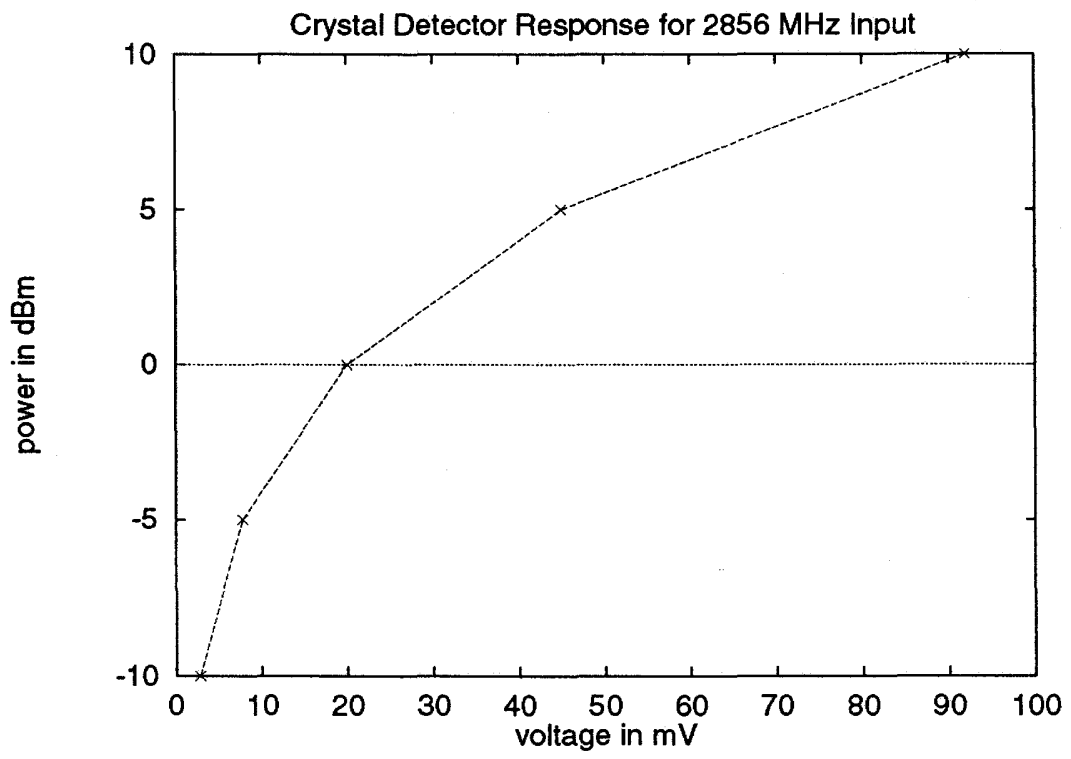

Figure 4.4: Response of Detector for First Harmonic

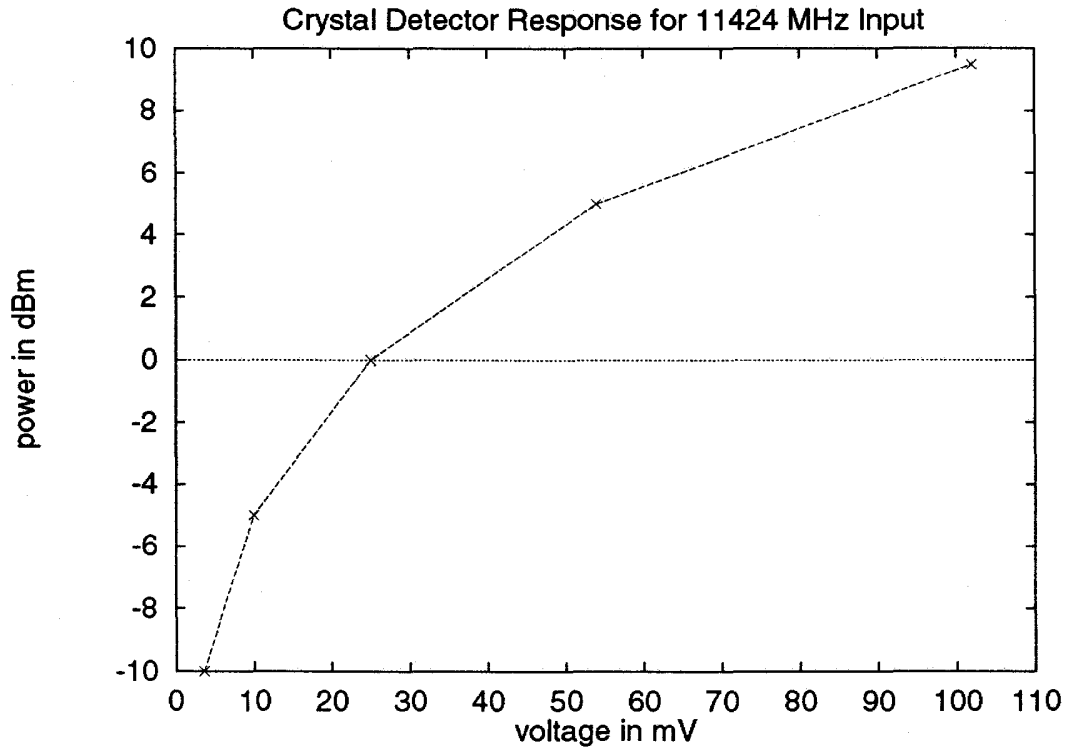

Figure 4.5: Response of Detector for Fourth Harmonic 


\subsubsection{The Bench Measurements}

The test setup consisted of the components labeled in Figure 4.6, which include a signal generator (HP 8672A), switch circuit, amplifier (Minicircuits ZHL-42), circulator (Huggins HC-7240), Step Recovery Diode (SRD) circuit and Test Set Output (TSO). Each component will be discussed concerning the function it performs.

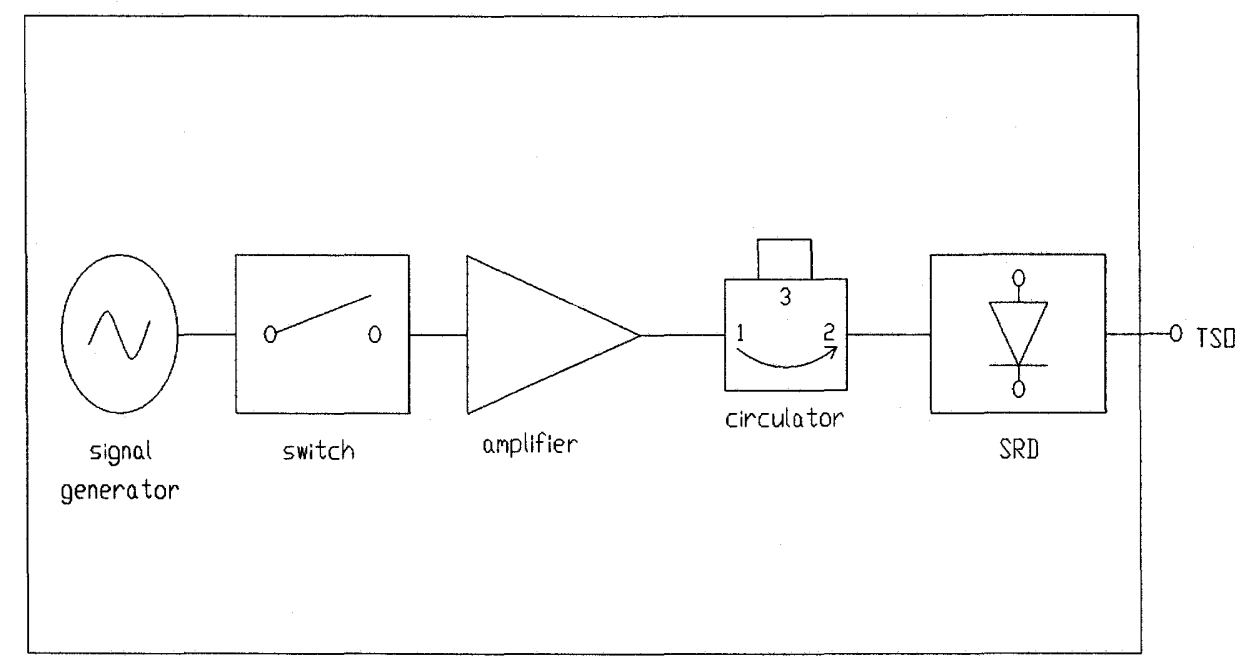

Figure 4.6: Test Setup

The synthesized signal generator provides a variable sinusoidal output from 1 to $18 \mathrm{GHz}$. The output power can be adjusted from -20 to $+10 \mathrm{dbm}$.

Figure 4.7 is the block diagram of the switch which consists of a 555 timer, multivibrator, an ECL-TTL translator and M/A-COM SW-328 SPDT switch.

The discharge, threshold and trigger pins on the timer are biased to have an astable mode of operation [27]. The frequency was chosen to mimic a possible injector pulse of $360 \mathrm{~Hz}$. The pulse width was $1.5 \mathrm{~ms}$, with rising and falling edges of $200 \mathrm{~ns}$. The timer is biased so that the pulse train has negative pulses from 0 to $-5 \mathrm{~V}$.

The multivibrator is set in monostable mode and uses the output of the timer as its trigger. The multivibrator utilizes a Schmitt trigger to provide insensitivity to variations in rising and falling edges from the timer [21]. The multivibrator operates 
on ECL logic levels, therefore the 0 to $-5 \mathrm{~V}$ logic levels of the timer are voltage divided to provide ECL logic levels of $-0.8 \mathrm{~V}$ for a $\mathrm{HI}$ and $-1.8 \mathrm{~V}$ for a LOW. External biasing sets the pulse width to $1 \mu \mathrm{s}$.

The next chip is the ECL-TTL translator. This is necessary since the GaAs switch is designed to work on voltage levels of 0 to $-5 \mathrm{~V}$ [19]. The translator output is biased to provide these levels.

The SW-328 acts as a switch for the input RF. From the RF input when the switch is $\mathrm{ON}$ or closed, the $\mathrm{RF}$ is allowed passage through the chip at $1 \mathrm{db}$ insertion loss to the TSO. When the switch is OFF, $30 \mathrm{db}$ of isolation is obtained [19].

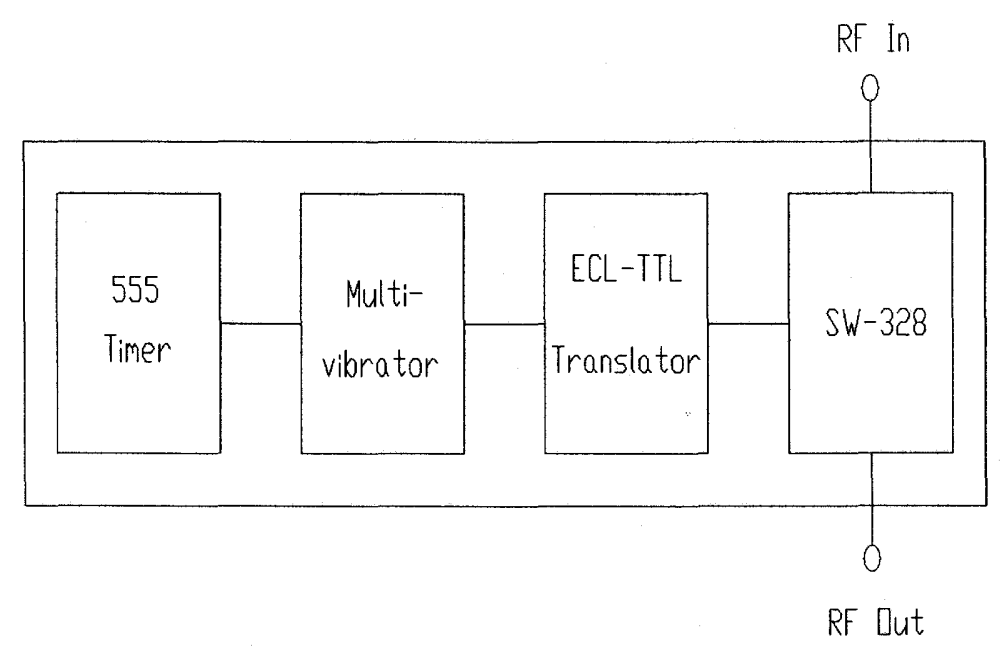

Figure 4.7: Switch Circuit

The amplifier increases the input power from $\approx 10 \mathrm{dbm}$ to $\approx 30 \mathrm{dbm}$. The circulator was included to protect the switch and amplifier from reflected power.

Finally, the SRD M/A-COM 43004-91 was used to provide a strong fourth harmonic component to the TSO and is illustrated in Figure 4.8. When the input RF is driving the SRD into forward conduction, it is in its low impedance state and stores charge. When the SRD is in reverse conduction, the stored charge is removed, thereby committing the SRD to its high impedance state [1]. The output waveform is illustrated in Figure 4.9. 


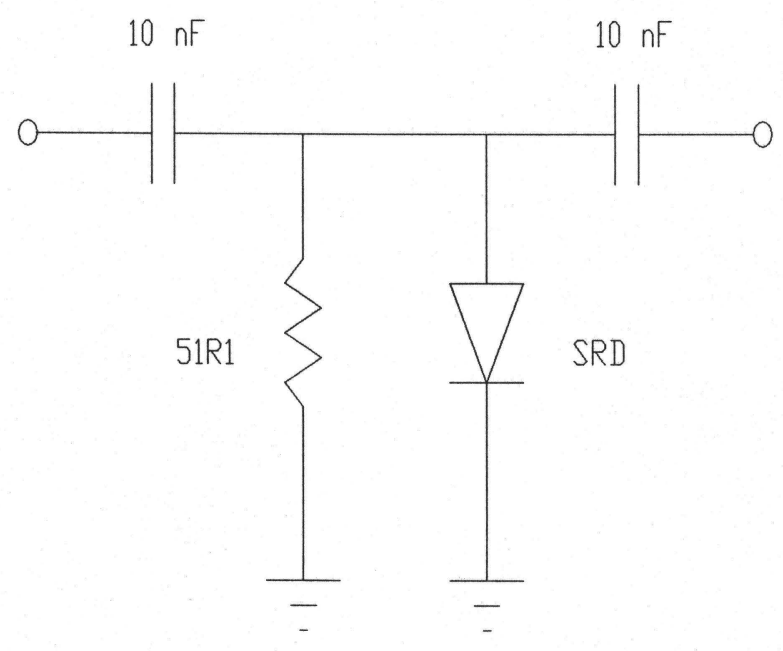

Figure 4.8: SRD Circuit

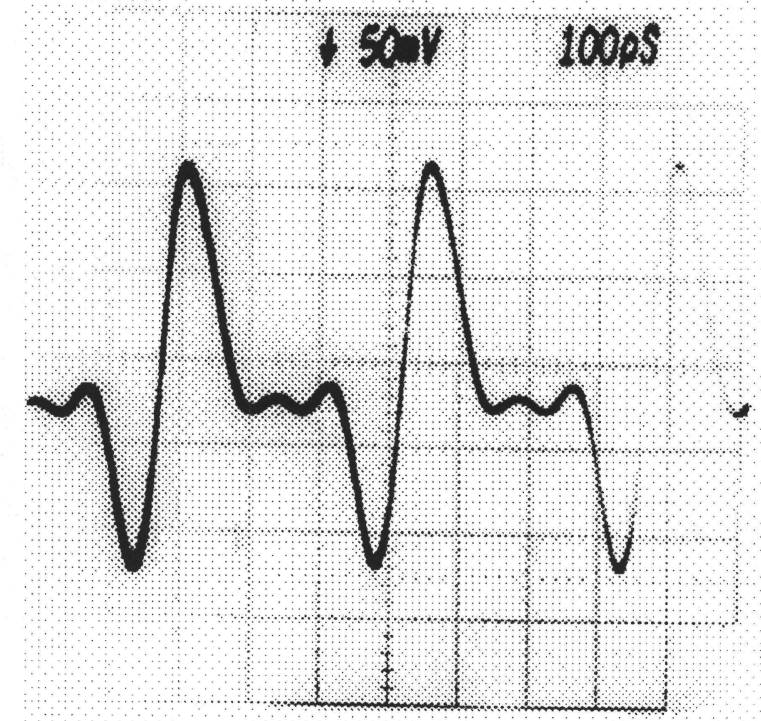

Figure 4.9: SRD Output 
Output Power

The HP 8990A Peak Power Meter (PPM) was connected to the TSO of Figure 4.6. The raw peak output power from the TSO was $18 \mathrm{~mW}$. By utilizing the filters, the output power for the first harmonic was $12 \mathrm{~mW}$ and for the fourth harmonic, $68 \mu \mathrm{W}$.

The next measurement involves the monitor with the $0.5 \mathrm{~cm}$ diameter button installed and a $50 \Omega$ termination on the output of the button port connected. Stub tuners were connected at the input and output plates of the monitor pipe to provide a better impedance match to the test setup [24]. The $11424 \mathrm{MHz}$ filter was connected to the pipe output and the tuners were optimized so that the fourth harmonic power reached a peak of $23 \mu \mathrm{W}$. The stub tuners were left in this state and the $2856 \mathrm{MHz}$ filter was connected to the pipe output which had $300 \mu \mathrm{W}$ of power. The total power out was $850 \mu \mathrm{W}$.

Finally, with the PPM sensor and the $50 \Omega$ termination from the button port exchanged, the total, first and fourth harmonic output power was obtained from each button. The values for each measurement are included in Table 4.1. Appendix C contains the scans of the pictures of the PPM display from which the values of Table 4.1 were obtained.

Table 4.1: Summary of Button Outputs

\begin{tabular}{|c|c|c|c|c|}
\hline Dimensions & Area & \multicolumn{3}{|c|}{ Output Power $(\mu \mathrm{W})$} \\
\hline Diameter $(\mathrm{cm})$ & $\left(\mathrm{cm}^{2}\right)$ & Total & First Harmonic & Fourth Harmonic \\
\hline 0.5 & 0.196 & 17.0 & 3.50 & 2.37 \\
\hline 1.0 & 0.785 & 34.0 & 14.5 & 1.50 \\
\hline 2.2 & 3.80 & 57.0 & 28.5 & 0.509 \\
\hline Length $(\mathrm{cm})$ & & & & \\
\hline 0.5 & 0.250 & 24.0 & 4.50 & 3.65 \\
\hline 1.0 & 1.00 & 40.0 & 15.7 & 2.25 \\
\hline 2.0 & 4.00 & 30.0 & 19.2 & 0.702 \\
\hline
\end{tabular}

It is clear from the data of Table 4.1 that the size (area) of the button is inversely proportional to the power coupled out at the fourth harmonic. This is an example of 
how a larger RC time constant results in a loss of time information by smoothing out the input pulse.

\subsection{Experimental Beam Trials}

Three experimental trials were conducted on the button monitor when it was installed in the beam line. These occurred on April $10^{\text {th }}$, July $10^{\text {th }}$ and August $10^{\text {th }} 1995$. Since each was unique, a brief description of procedures and the output data will be done separately.

For each test run, the $1 \mathrm{~cm}$ diameter button was used. It was found to have the best output power for both harmonics and did not have the sharp edges of the square button, which tend to accumulate charge [15]. After the button was installed, the monitor pipe was placed in the beam line and a $13.4 \mathrm{~m}$ length of Heliax cable was strung from the button port through an access hole in the Linac Vault to the Drive Room. From the Drive Room the cable continued to where a power divider was attached. The two signals are then fed into the two BP filters and then to the PPM. A NeXT workstation was used in the test area which had a GPIB cable connected from the PPM to the NeXT to allow for data transfer. Figure 4.10 illustrates the layout common for each run.

\subsubsection{April Run}

With Section \#1 phase set at $74^{\circ}$, Section \#2 transmitter off and the prebuncher phase at $29^{\circ}$, the measurement procedure began by saving this button output as displayed by the PPM. Then the prebuncher phase was changed, which directly affected the bunching, and the stored data were then subtract from each succeeding measurement. A definitive number for the output power for each of the three trials were obtained by averaging the last 300 ns of the output pulse at each prebuncher phase value.

In Table 4.2 , it is the change in output power that is recorded, with the exception 


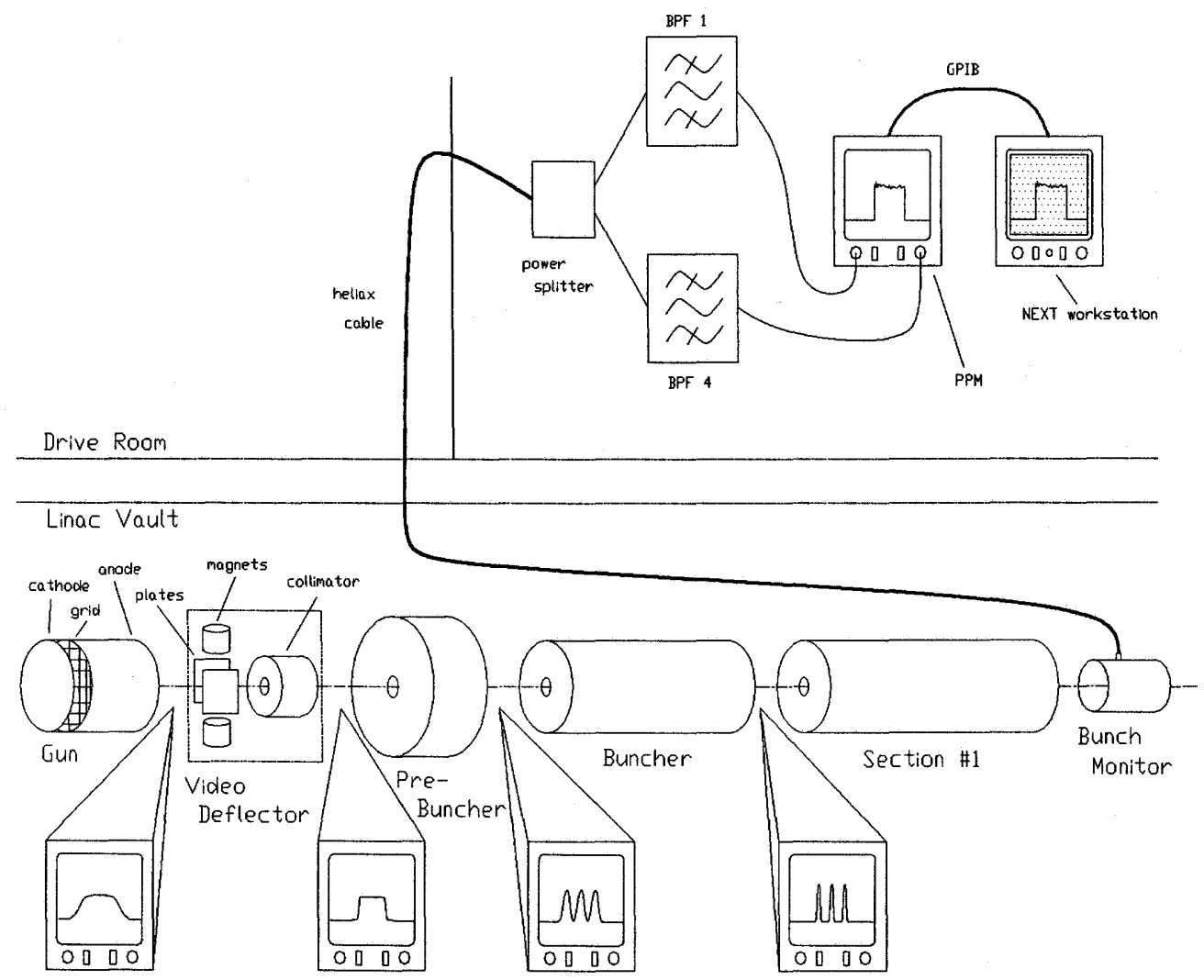

Figure 4.10: Experimental Test Setup

of the first absolute measurement.

The Heliax cable attenuated the first and fourth harmonics differently [2]. The cable attenuation was $1.94 \mathrm{db}$ for the first harmonic and $4.25 \mathrm{db}$ for the fourth harmonic. This factor is calculated into the Actual Output Power column in Table 4.2. The operator phase numbers should be multiplied by 1.72 to get the actual relative phase from succeeding measurements. The toroid current is measured at a toroid located just after the button monitor but before Section \#2. It measures a voltage from the toroid which has been calibrated to provide the average current over the RF pulse width [4]. The plots that the data in Table 4.2 were derived from are included in Appendix D. Figure 4.11 is a graphical representation of the voltage ratio 
in Table 4.2 versus prebuncher phase. For these data and subsequent data, error measurements are not included but are assumed to be constant for each data point for each test run.

Table 4.2: Summary of Button Output for April Test Run

\begin{tabular}{|c|c|c|c|c|c|c|}
\hline $\begin{array}{c}\text { Operator } \\
\text { Phasing }\left(^{\circ}\right)\end{array}$ & $\begin{array}{c}\text { Toroid } \\
\text { Current }(\mathrm{mA})\end{array}$ & \multicolumn{2}{|c|}{$\begin{array}{c}\text { Raw Output } \\
\text { Power }(\mu \mathrm{W})\end{array}$} & \multicolumn{2}{c|}{$\begin{array}{c}\text { Actual Output } \\
\text { Power }(\mu \mathrm{W})\end{array}$} & $V_{\mathbf{1}}$ \\
\hline & & First & Fourth & First & Fourth & \\
\hline 29 & 100 & 636 & 1850 & 995 & 4930 & 0.4490 \\
\hline 19 & 95 & -12.3 & -1.05 & -19.3 & -2.80 & 0.4447 \\
\hline 10 & 93 & -37.2 & -132 & -58.2 & -350 & 0.4520 \\
\hline 29 & 100 & -7.83 & -45.0 & -12.2 & -120 & 0.4517 \\
\hline 39 & 100 & -5.29 & -143 & -8.28 & -379 & 0.4653 \\
\hline 49 & 95 & -138 & -279 & -216 & -742 & 0.4309 \\
\hline 59 & 86 & -293 & -767 & -458 & -2040 & 0.4306 \\
\hline 69 & 57 & -452 & -1380 & -707 & -3670 & 0.4773 \\
\hline 79 & 33 & -545 & -1550 & -852 & -4130 & 0.4206 \\
\hline 29 & 100 & -6.72 & -48.6 & -10.5 & -129 & 0.4526 \\
\hline
\end{tabular}

\section{Variation of Test Setup}

The second phase of this run was a "proof of principle" of the ability of the monitor to make bunch measurements in a configuration close to its final form. The test assembly was changed to have the power splitter and filters connected directly to the button port with the crystal diode detectors connected to the filters. RG58 coaxial cables were laid on the same path as the Heliax through the Drive Room and on to the test area. A digital scope was used to observe the signals with GPIB interface to the NeXT. Scope displays were saved in encapsulated postscript.

To eliminate the transmitter noise, the $\mathrm{ON}$ and OFF triggers for the video deflector were made to coincide in time, allowing no beam to pass through the injector. In this way, the transmitter noise could be saved in the scope memory and subtracted from subsequent measurements. 


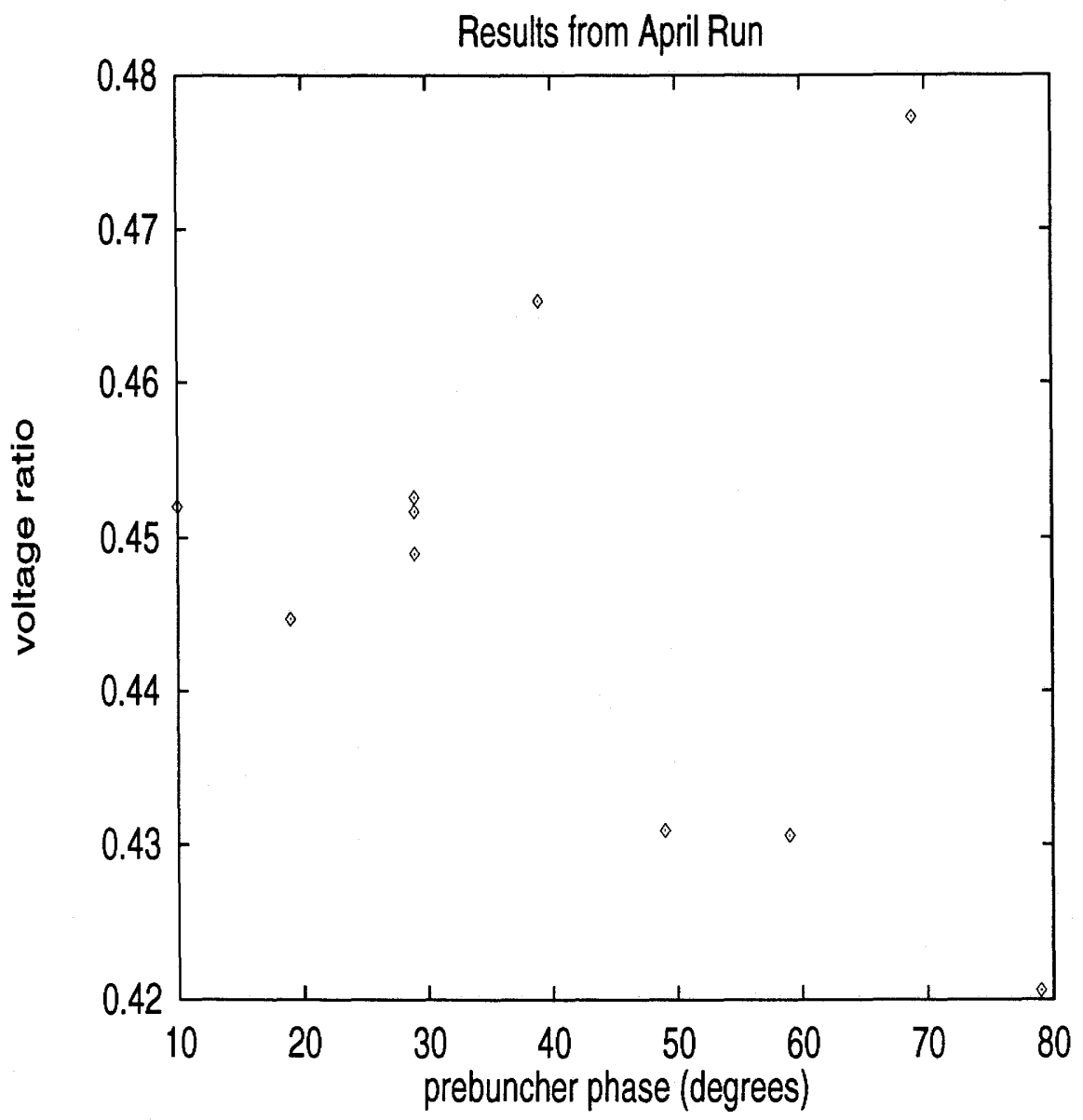

Figure 4.11: April Results:Experimental Dependence of Prebuncher Phase on Bunch Width

Starting from the arbitrary $29^{\circ}$ prebuncher phase and then varying it on either side, output obtained from the scope traces are illustrated in Appendix E and recorded in Table 4.3 by using the crystal detector calibration curves of Figures 4.4 and 4.5 .

Although the magnitude of the ratios in Table 4.3 are in general somewhat larger than that for Table 4.2, the relative changes follow the same trend. This is confirmation that this setup configuration will provide the desired sensitivity. Figure 4.12 is an example of the output of the detectors at prebuncher phase of $29^{\circ}$ and after subtracting the transmitter noise. The top trace is the first harmonic and the bottom is the fourth. 
Table 4.3: Summary of Button Output from Crystal Detectors

\begin{tabular}{|c|c|c|c|c|}
\hline Prebuncher & Toroid & \multicolumn{2}{|c|}{ Output Voltage $(\mathrm{mV})$} & \multirow{2}{*}{$\frac{P_{1}}{P_{4}}$} \\
\cline { 2 - 4 } Phasing $\left(^{\circ}\right)$ & Current $(\mathrm{mA})$ & First Harmonic & Fourth Harmonic & \\
\hline 29 & 102 & 15 & 43 & 0.610 \\
\hline 10 & 95 & 15 & 45 & 0.664 \\
\hline 49 & 105 & 13 & 37 & 0.584 \\
\hline 69 & 59 & 5 & 13 & 0.645 \\
\hline
\end{tabular}

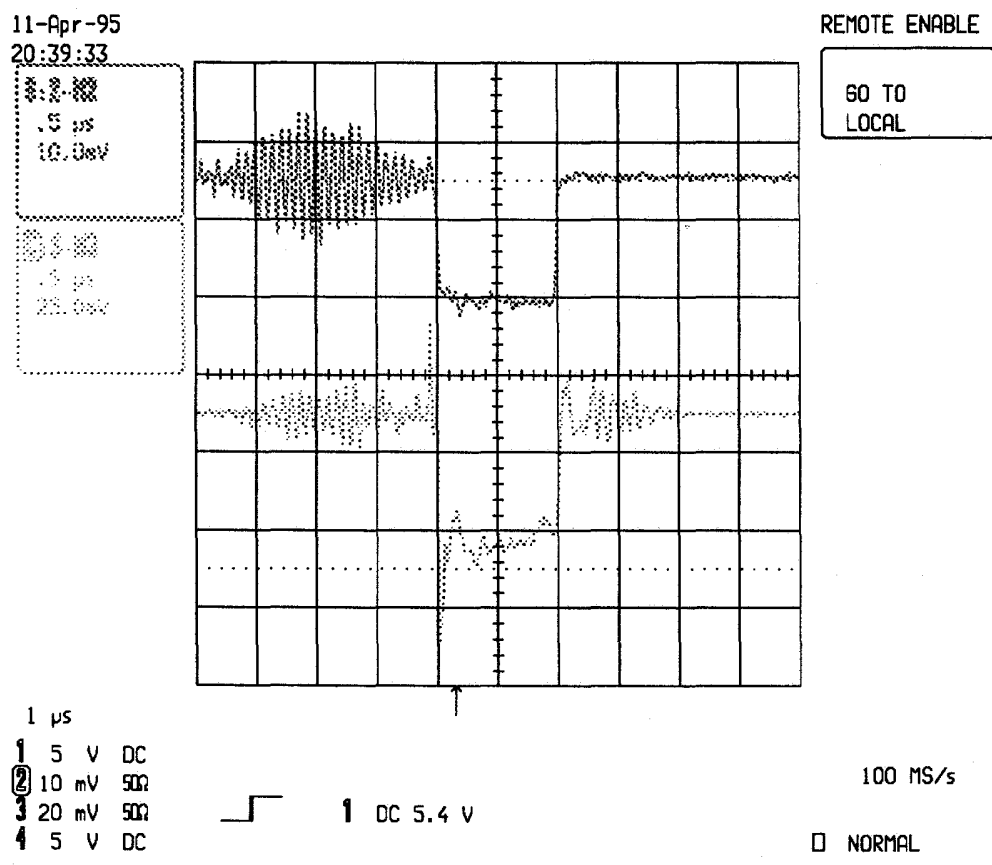

Figure 4.12: Output of Monitor after Noise Subtraction

It was decided that another run would be required to obtain more data and confirm the April results.

\subsubsection{July Run}

The experimental setup for this run was identical for that of the April $10^{\text {th }}$ run except in one respect - the transmitters for Sections \#2 - \#6 were also running. This change would create havoc with the interpretation of the results since the noise from the button would be much greater. 


\section{Noise Discussion}

With reference to Figures 4.13 and 4.14 two facts can be noted. First, that the magnitude of the power output is greater for Figure 4.14, and that it also has noise in the vicinity of the beam signal. These are plots from the April $10^{\text {th }}$ run and correspond to the first row of data in Table 4.2 .

The plots from the July $10^{\text {th }}$ run are shown in Figures 4.15 and 4.16 . It is clear that the output at the fourth harmonic is very much larger than that for the April run. To understand why, refer to Figure 4.17 which is a close-up of the structures between the two sections.

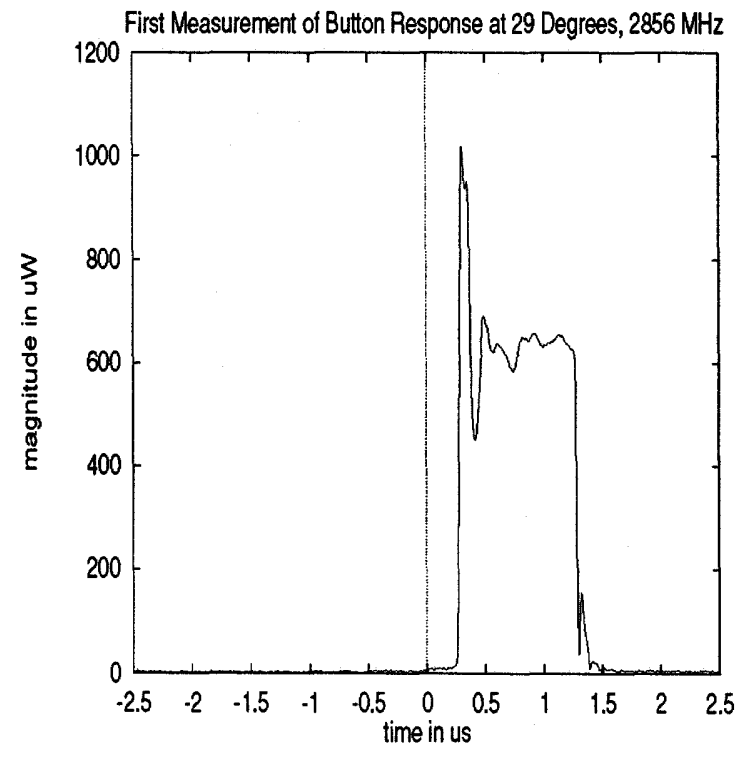

Figure 4.13: April Result: First Harmonic Response with Prebuncher Phase $29^{\circ}$

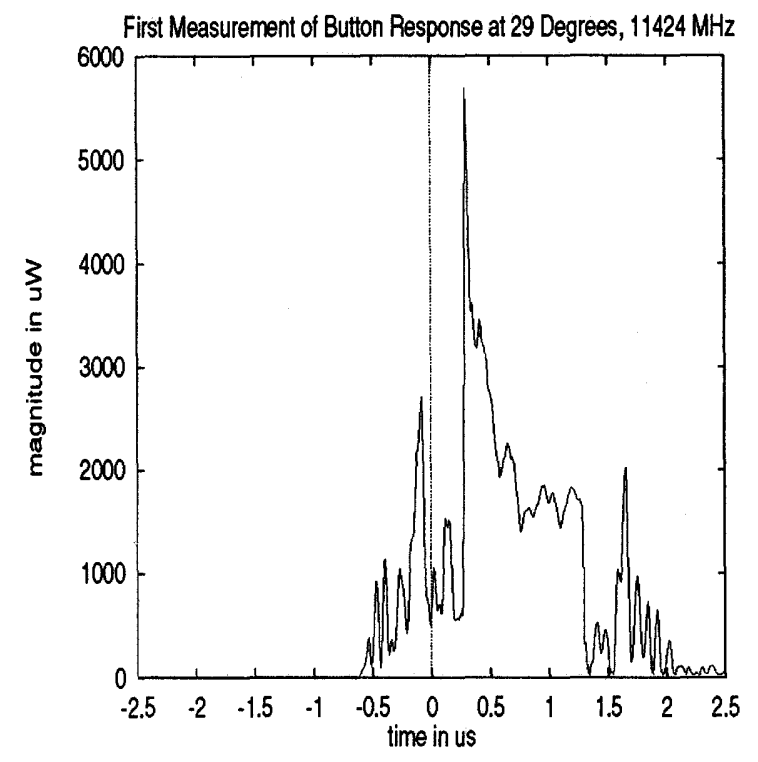

Figure 4.14: April Result: Fourth Harmonic Response with Prebuncher Phase $29^{\circ}$ 


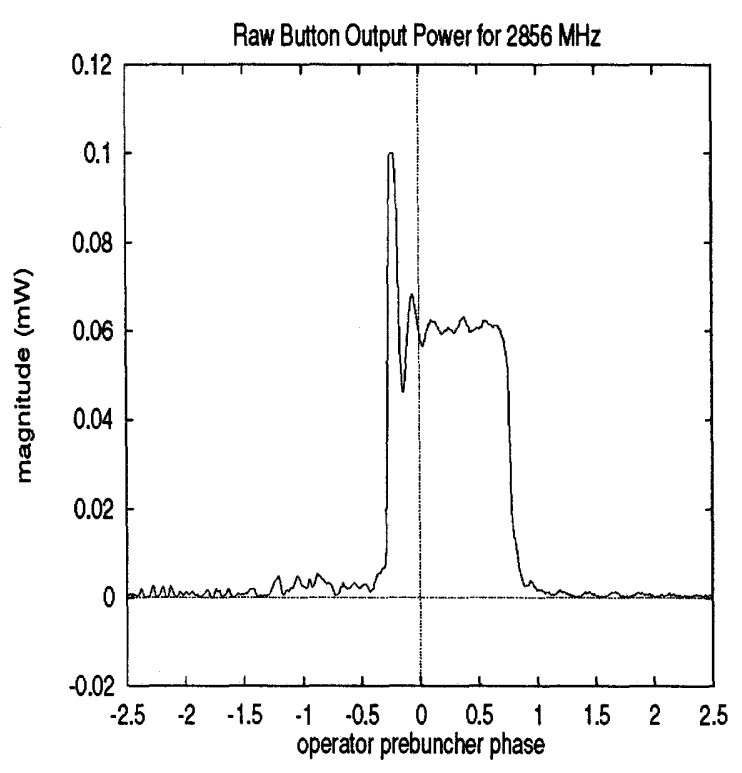

Figure 4.15: July Result: First Harmonic Response with Prebuncher Phase $29^{\circ}$

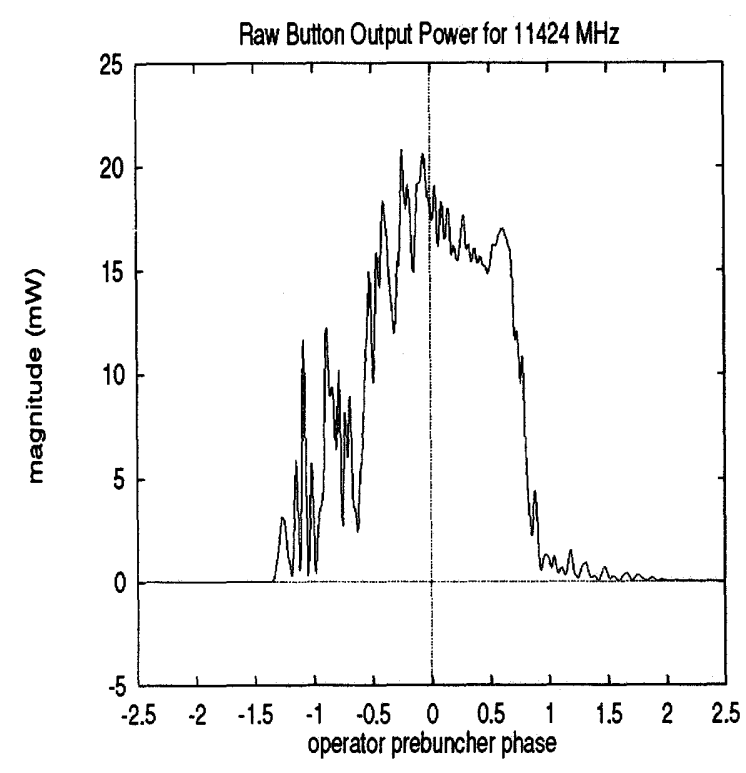

Figure 4.16: July Result: Fourth Harmonic Response with Prebuncher Phase $29^{\circ}$

In the April run, microwave power was only available from Section \#1. After it travels through Section \#1, it proceeds into an output coupler and provides power for Section \#7. Since all sections are essentially bandpass filters [22], the output of higher harmonics at the exit of Section \#1 is very small. The stray fields of the first harmonic that continue past the output coupler are quickly attenuated by the $6 \mathrm{~cm}$ diameter beam pipe, which has a cutoff frequency of $3.83 \mathrm{GHz}$ [24] and is essentially a highpass filter.

This situation is different for the input coupler of Section \#2. The first harmonic power cannot travel back toward Section \#1 because of the beam pipe, although the fourth harmonic can. With $20 \mathrm{MW}$ of microwave power located only $170 \mathrm{~cm}$ from the monitor, it is easy to imagine a large amount of power being coupled to the button at the fourth harmonic.

Indeed, this is the case. In the August run, the transmitter for Section \#2 was turned on and a large peak of noise similar to Figure 4.14 was observed. With this transmitter off, a clean and strong signal was obtained.

With this explanation in mind, the noise power was measured to be $32 \mathrm{~mW}$ on 


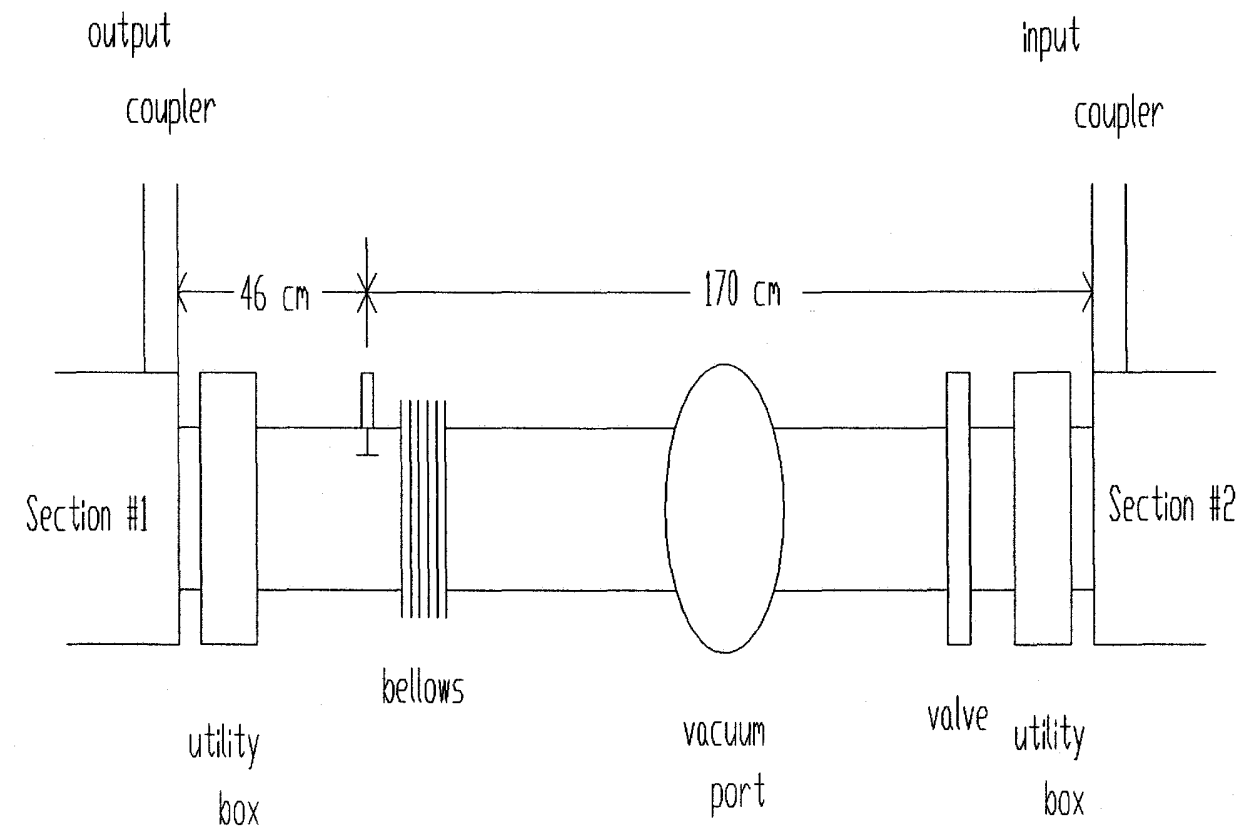

Figure 4.17: Components Between Section \#1 and Section \#2

July $11^{\text {th }}$. The data recorded in Table 4.4 has the noise offset subtracted from the button output. 
Table 4.4: Summary of Button Output for July Test Run

\begin{tabular}{|c|c|c|c|c|c|c|}
\hline $\begin{array}{c}\text { Operator } \\
\text { Phase }\left(^{\circ}\right)\end{array}$ & $\begin{array}{c}\text { Toroid } \\
\text { Current }(\mathrm{mA})\end{array}$ & \multicolumn{2}{|c|}{$\begin{array}{c}\text { Raw Output } \\
\text { Power }(\mu \mathrm{W})\end{array}$} & \multicolumn{2}{|c|}{$\begin{array}{c}\text { Actual Output } \\
\text { Power }(\mu \mathrm{W})\end{array}$} & $\frac{V}{1}_{\mathbf{4}}$ \\
\hline & & First & Fourth & First & Fourth & \\
\hline 23 & 32 & 95.2 & 10600 & 149 & 28200 & 0.07269 \\
\hline 18 & 31 & 96.7 & 10100 & 151 & 26900 & 0.07492 \\
\hline 13 & 31 & 97.6 & 9980 & 153 & 26600 & 0.07584 \\
\hline 9 & 30 & 97.5 & 9440 & 152 & 25100 & 0.07782 \\
\hline 23 & 32 & 95.4 & 8880 & 149 & 23600 & 0.07524 \\
\hline 28 & 31 & 94.3 & 9660 & 147 & 25700 & 0.07563 \\
\hline 33 & 31 & 92.7 & 10300 & 145 & 27400 & 0.07275 \\
\hline 38 & 30 & 91.1 & 11100 & 142 & 29500 & 0.06938 \\
\hline 43 & 30 & 88.5 & 11000 & 138 & 29300 & 0.06862 \\
\hline 48 & 29 & 83.5 & 10100 & 131 & 26900 & 0.06978 \\
\hline 53 & 27 & 73.0 & 7500 & 114 & 20000 & 0.07550 \\
\hline 58 & 25 & 62.8 & 4850 & 98.2 & 12900 & 0.08725 \\
\hline 63 & 15 & 45.2 & 2470 & 70.7 & 6570 & 0.1037 \\
\hline 68 & 12 & 25.6 & 4660 & 40.0 & 12400 & 0.05680 \\
\hline 73 & 11 & 21.9 & 5050 & 34.2 & 13400 & 0.05052 \\
\hline 78 & 10 & 20.0 & 4800 & 31.3 & 12800 & 0.04945 \\
\hline 23 & 32 & 96.2 & 9960 & 150 & 26500 & 0.07523 \\
\hline
\end{tabular}




\section{Energy Deviation}

The July run was set up to prove that prebuncher phase is directly proportional to the energy spread. The procedure started with an arbitrary phase for the prebuncher and a magnet bent the electron path at the end of the Linac through a narrow slit and into a large iron block with an ammeter attached for current measurement.

The peak of the current pulse is displayed in the control room by varying the magnetic field of the magnet. Once this peak is found, the magnetic field is varied to reduce the current pulse to half its maximum on both sides of the peak. Another display relates the strength of the magnetic field to the electron energy. Table 4.5 contains the results of this procedure.

Figure 4.18 illustrates the first-to-fourth voltage ratio versus prebuncher phase of Table 4.4. Figure 4.19 is a plot of the energy difference versus prebuncher phase of Table 4.5. The two curves are plotted on the same prebuncher phase scale to illustrate that the minimum in voltage ratio or bunch width corresponds approximately to the narrowest energy spread.

Table 4.5: Energy Spread of Beam

\begin{tabular}{|c|c|c|c|c|c|}
\hline $\begin{array}{c}\text { Prebuncher } \\
\text { Phase }\left({ }^{\circ}\right)\end{array}$ & $\begin{array}{c}\text { Peak } \\
\text { Energy }(\mathrm{MeV})\end{array}$ & $\begin{array}{c}\text { Half Current } \\
\text { Energy }(\mathrm{MeV})\end{array}$ & $\begin{array}{c}\text { Half Current } \\
\text { Energy }(\mathrm{MeV})\end{array}$ & $\begin{array}{c}\text { Difference } \\
(\mathrm{MeV})\end{array}$ & $\begin{array}{c}\text { \% of } \\
\text { Peak }\end{array}$ \\
\hline 11 & 264.705 & 262.895 & 266.780 & 3.89 & 1.47 \\
\hline 30 & 265.000 & 262.699 & 266.519 & 3.85 & 1.45 \\
\hline 53 & 264.116 & 266.151 & 267.806 & 3.69 & 1.39 \\
\hline 70 & 269.083 & 266.684 & 270.753 & 4.07 & 1.51 \\
\hline
\end{tabular}




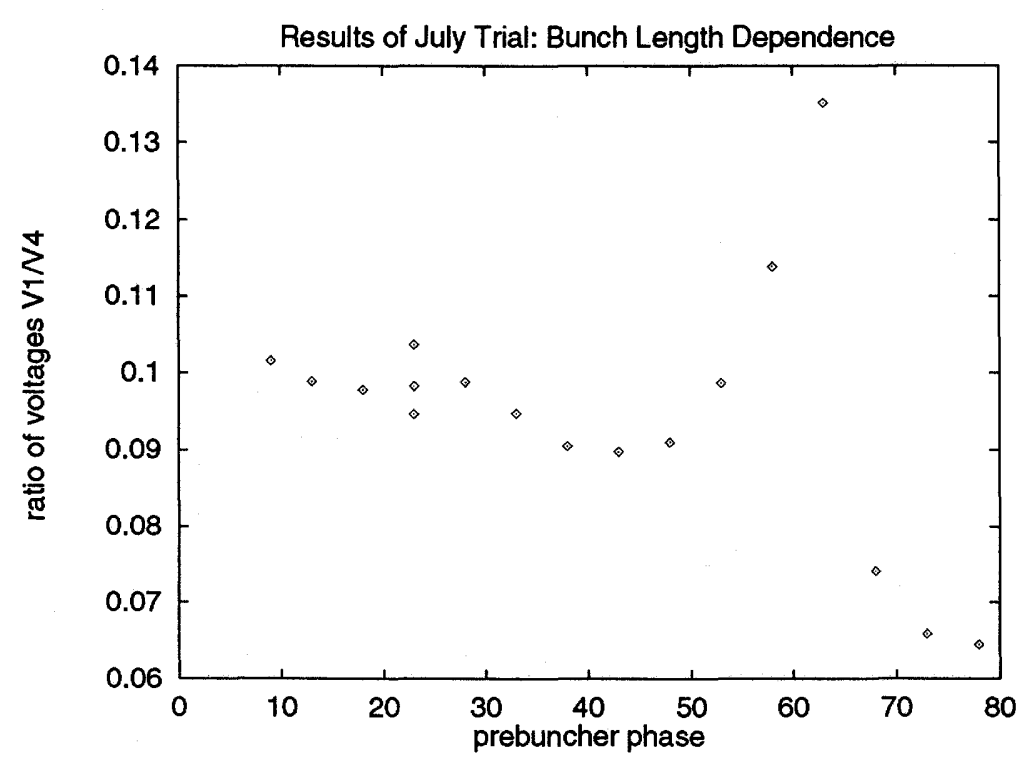

Figure 4.18: July Results: Experimental Dependence of Bunch Width on Prebuncher Phase

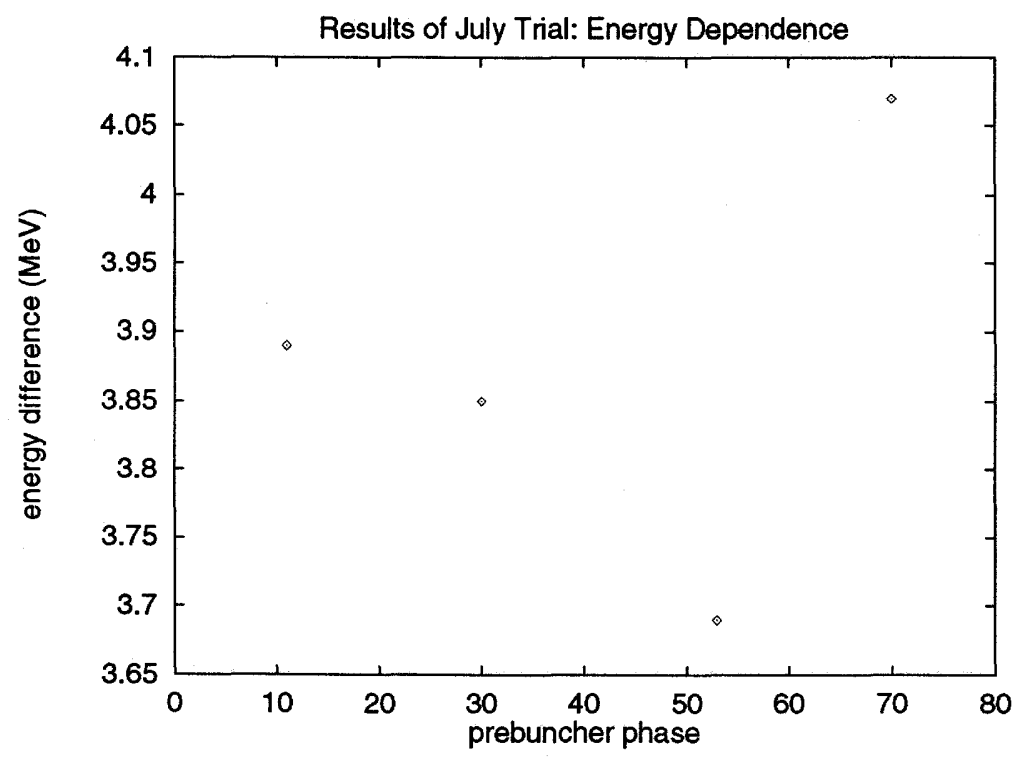

Figure 4.19: July Results: Experimental Dependence of Beam Energy on Prebuncher Phase 


\subsubsection{August Run}

The August run was identical to the April run, except that the PPM display was used to average the button pulse. As a result, the NeXT and GPIB interface was not required. With Section \#2 transmitter off, the output was a very clean signal, much cleaner than found in the April run.

Two experiments were done, one with Section \#1 phase at $69^{\circ}$ and the other with Section \#1 at $75^{\circ}$. The data is recorded in Tables 4.6 and 4.7 and plotted in Figures 4.20 and 4.21 .

Figures 4.20 and 4.21 illustrate the effect of the prebuncher phase and Section \#1 phase moving relative to one another. The bunch length minima from the Injector and Section \#1 are clearly visible and appear to become more defined for greater Section \#1 phase values.

Table 4.6: Summary of Button Output for August Run, Section \#1 Phase $69^{\circ}$

\begin{tabular}{|c|c|c|c|c|c|c|}
\hline $\begin{array}{c}\text { Operator } \\
\text { Phasing }\left(^{(}\right)\end{array}$ & $\begin{array}{c}\text { Toroid } \\
\text { Current }(\mathrm{mA})\end{array}$ & \multicolumn{2}{|c|}{$\begin{array}{c}\text { Raw Output } \\
\text { Power }(\mu \mathrm{W})\end{array}$} & \multicolumn{2}{|c|}{$\begin{array}{c}\text { Actual Output } \\
\text { Power }(\mu \mathrm{W})\end{array}$} & $\bar{V}_{V_{4}}$ \\
\hline & & First & Fourth & First & Fourth & \\
\hline 29 & 96 & 578 & 4650 & 904 & 12400 & 0.2702 \\
\hline 24 & 94 & 563 & 4500 & 880 & 11200 & 0.2711 \\
\hline 19 & 82 & 542 & 4110 & 847 & 10900 & 0.2783 \\
\hline 14 & 74 & 513 & 3570 & 802 & 9500 & 0.2906 \\
\hline 10 & 70 & 504 & 3390 & 788 & 9020 & 0.2955 \\
\hline 29 & 100 & 572 & 4600 & 894 & 12200 & 0.2703 \\
\hline 34 & 101 & 538 & 4300 & 841 & 11400 & 0.2711 \\
\hline 39 & 90 & 392 & 3000 & 613 & 7980 & 0.2771 \\
\hline 44 & 79 & 312 & 2280 & 488 & 6070 & 0.2835 \\
\hline 49 & 71 & 252 & 1750 & 394 & 4660 & 0.2908 \\
\hline 54 & 65 & 217 & 1490 & 339 & 3960 & 0.2925 \\
\hline 59 & 59 & 183 & 1220 & 286 & 3250 & 0.2969 \\
\hline 64 & 53 & 152 & 927 & 238 & 2470 & 0.3104 \\
\hline 69 & 46 & 121 & 625 & 189 & 1660 & 0.3373 \\
\hline 74 & 41 & 92.0 & 386 & 144 & 1030 & 0.3742 \\
\hline 79 & 37 & 66.3 & 160 & 104 & 426 & 0.4934 \\
\hline 29 & 100 & 578 & 4640 & 904 & 12300 & 0.2705 \\
\hline & & & & & & \\
\hline
\end{tabular}


Table 4.7: Summary of Button Output for August Run, Buncher Phase $75^{\circ}$

\begin{tabular}{|c|c|c|c|c|c|c|}
\hline $\begin{array}{c}\text { Operator } \\
\text { Phasing }\left(^{\circ}\right)\end{array}$ & $\begin{array}{c}\text { Toroid } \\
\text { Current }(\mathrm{mA})\end{array}$ & \multicolumn{2}{|c|}{$\begin{array}{c}\text { Raw Output } \\
\text { Power }(\mu \mathrm{W})\end{array}$} & \multicolumn{2}{|c|}{$\begin{array}{c}\text { Actual Output } \\
\text { Power }(\mu \mathrm{W})\end{array}$} & $\frac{V_{1}}{V_{4}}$ \\
\hline & & First & Fourth & First & Fourth & \\
\hline 29 & 104 & 564 & 5170 & 882 & 13800 & 0.2532 \\
\hline 24 & 98 & 566 & 5080 & 885 & 13500 & 0.2559 \\
\hline 19 & 87 & 546 & 4660 & 854 & 12400 & 0.2624 \\
\hline 14 & 81 & 519 & 4070 & 811 & 10830 & 0.2737 \\
\hline 10 & 81 & 510 & 3840 & 797 & 10220 & 0.2793 \\
\hline 29 & 104 & 563 & 5150 & 880 & 13700 & 0.2534 \\
\hline 34 & 101 & 520 & 4690 & 813 & 12500 & 0.2552 \\
\hline 39 & 90 & 371 & 3160 & 580 & 8410 & 0.2626 \\
\hline 44 & 81 & 302 & 2460 & 472 & 6550 & 0.2686 \\
\hline 49 & 74 & 258 & 2060 & 403 & 5480 & 0.2713 \\
\hline 54 & 69 & 222 & 1790 & 347 & 4760 & 0.2699 \\
\hline 59 & 63 & 186 & 1540 & 291 & 4100 & 0.2664 \\
\hline 64 & 56 & 153 & 1240 & 239 & 3300 & 0.2692 \\
\hline 69 & 50 & 126 & 990 & 197 & 2630 & 0.2734 \\
\hline 74 & 42 & 100 & 672 & 156 & 1790 & 0.2957 \\
\hline 79 & 37 & 76.0 & 425 & 119 & 1130 & 0.3241 \\
\hline 29 & 104 & 558 & 5100 & 873 & 13600 & 0.2535 \\
\hline
\end{tabular}




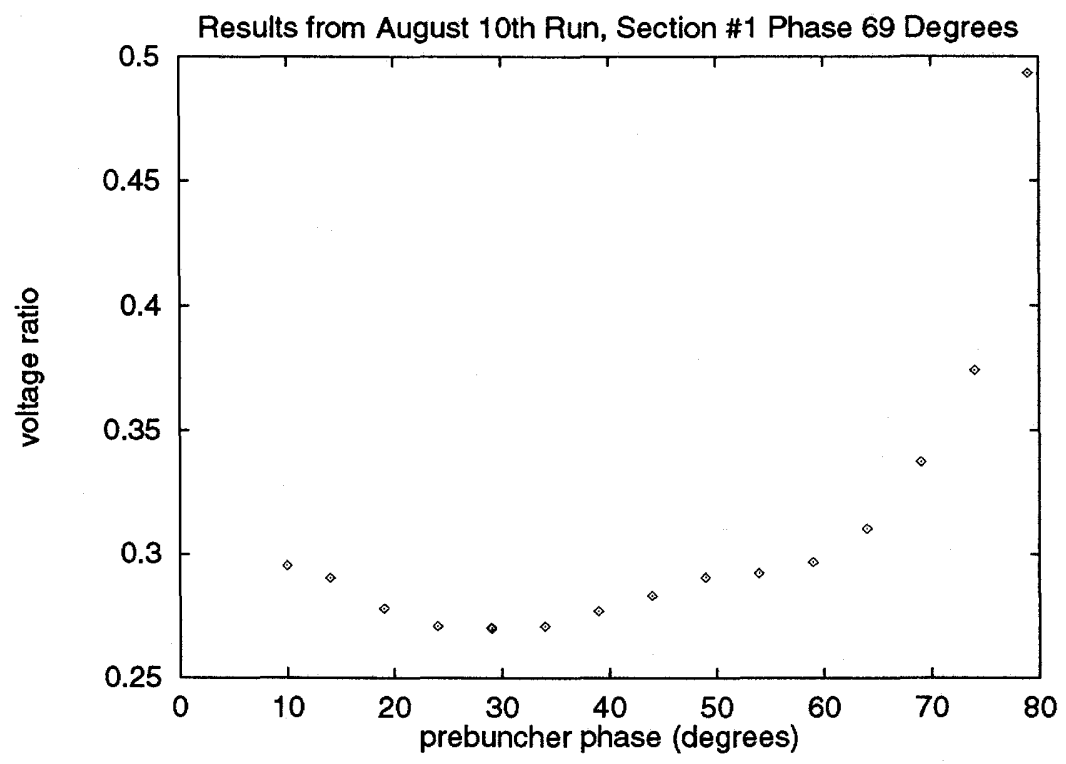

Figure 4.20: August Results:Experimental Dependence of Prebuncher Phase on Bunch Width for Section \#1 Phase of $69^{\circ}$

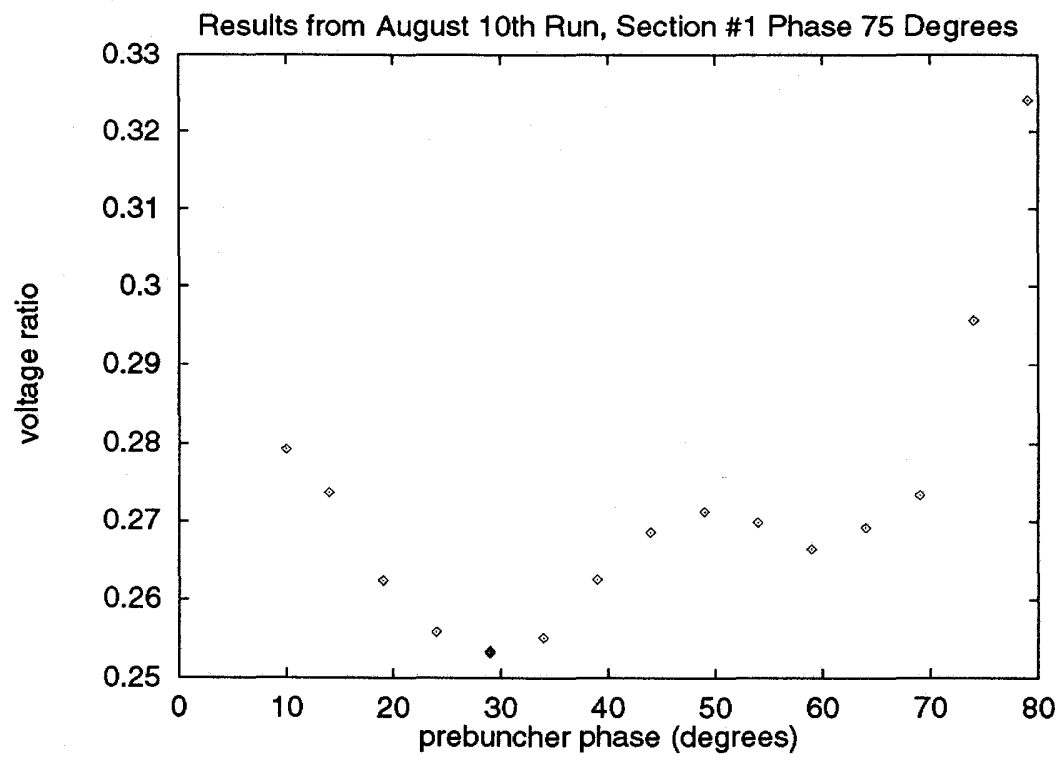

Figure 4.21: August Results:Experimental Dependence of Prebuncher Phase on Bunch Width for Section \#1 Phase of $75^{\circ}$ 


\section{Chapter 5}

\section{Summary and Conclusions}

The principle of monitoring the bunch length at the front end of the Linac has been discussed. It has been shown that a narrow bunch width provides a minimum in energy spread, which was the motivation to develop the monitor. A literature search indicated that the best monitor for the application would be a button monitor mounted as close as possible to the injector. Its operation relies on the principle that in the time domain, a Gaussian beam pulse with standard deviation $\sigma$ has in the frequency domain a Gaussian shape but with the standard deviation in the denominator of the argument. The effect is that a narrow pulse width in time is a broad pulse in frequency. By manipulating sufficiently wide harmonics, an indication of the bunch

width in time can be obtained. The monitor exploits this characteristic by detecting power at the first and fourth harmonics of the machine frequency and divides them to provide a ratio related to bunch length.

Computer simulations on the feasibility of using this monitor at SAL were favourably demonstrated, and it was decided to construct the monitor and install it just after Section \#1, as illustrated in Figure 1.2.

The bench tests revealed that a button of $1 \mathrm{~cm}$ diameter mounted $3 \mathrm{~mm}$ from the beam pipe wall would provide sufficient signal strength for signals to be brought upstairs. A power splitter and bandpass filters at $2856 \mathrm{MHz}$ and $11424 \mathrm{MHz}$ provided signals that are related to the first harmonic and the fourth.

After installation and three experimental runs, it has been proven that the monitor operates adequately. Clear minima can be seen in plots of voltage ratio versus prebuncher phase as shown in Figure 5.1. The two minima are due to the separate dependence of the injector and Section \#1 on energy spread and it is clear that one of the minima is an absolute minimum. 
As shown in Tables 4.5 and Table 5.1, by only changing the prebuncher phase, the percent energy spread of the beam can change from a value of $1.51 \%$ to a value of $1.39 \%$. With more rigorous optimization, a value of $1.30 \%$ or less could be realized.

Figure 5.2 illustrates the results from Table 5.1 in graphic form. It is plotted with the same horizontal scale to demonstrate the similarities in the curves. A clear minimum is observed about the $50^{\circ}$ position and a maximum at $\approx 68^{\circ}$ in both Figures 5.1 and 5.2 .

Table 5.1: Energy Spread of Beam

\begin{tabular}{|c|c|c|c|c|c|}
\hline $\begin{array}{c}\text { Prebuncher } \\
\text { Phase }\left(^{\circ}\right)\end{array}$ & $\begin{array}{c}\text { Peak } \\
\text { Energy }(\mathrm{MeV})\end{array}$ & $\begin{array}{c}\text { Half Current } \\
\text { Energy }(\mathrm{MeV})\end{array}$ & $\begin{array}{c}\text { Half Current } \\
\text { Energy }(\mathrm{MeV})\end{array}$ & $\begin{array}{c}\text { Difference } \\
(\mathrm{MeV})\end{array}$ & $\begin{array}{c}\text { \% of } \\
\text { Peak }\end{array}$ \\
\hline 11 & 264.705 & 262.895 & 266.780 & 3.89 & 1.47 \\
\hline 30 & 265.000 & 262.699 & 266.519 & 3.85 & 1.45 \\
\hline 53 & 264.116 & 266.151 & 267.806 & 3.69 & 1.39 \\
\hline 70 & 269.083 & 266.684 & 270.753 & 4.07 & 1.51 \\
\hline
\end{tabular}

The monitor worked well with the raw signal transported upstairs where it was split and sent to filters and a Peak Power Meter. By substituting crystal detectors for the Peak Power Meter and connecting them along with the bandpass filters and power splitter directly to the button port, it was measured that sensitivity of the monitor with the crystal detectors was similar to that of the Peak Power Meter. In the future, this assembly would provide the front end electronics for the monitor. A dedicated microprocessor with analog-to-digital conversion capability and on-board RAM should be installed to handle the data acquisition.

In its final form, the operator will start the bunch length minimization procedure at an arbitrary prebuncher and Section \#1 phase. It was intended that the monitor be used with the accelerator in operation and several suggestions on eliminating the transmitter noise from Section \#2 have been discussed. The most likely method would be to separate the Section \#2 transmitter pulse from the beam pulse in time so that the measurements can be done with a clean signal. This would cause less than a minute's delay in beam usage when the Section \#2 transmitter pulse would 
again accelerate electrons.

The data acquisition would use the trigger from the video deflector $\mathrm{ON}$ to provide timing. In order to allow transients in the beam pulse to decay and also to sample outside the filter time constants, a few hundred nanoseconds must elapse before the sampling begins. Several samples should be taken over the pulse, therefore a two port $\mathrm{A} / \mathrm{D}$ of $50 \mathrm{MHz}$ or greater is desired. The signals vary on the order of millivolts, so with no amplification the accuracy provided by a 12 bit $\mathrm{A} / \mathrm{D}$ is adequate.

After the values of the two harmonics have noise subtracted from them they will be divided and the ratio is sent to an LED display in the control room. The operator can minimize the ratio by changing the prebuncher phase. The video deflector OFF ends the acquisition cycle for each pulse.

In this way, rapid and accurate bunching efficiency can be judged with minimal demand on beam time. 


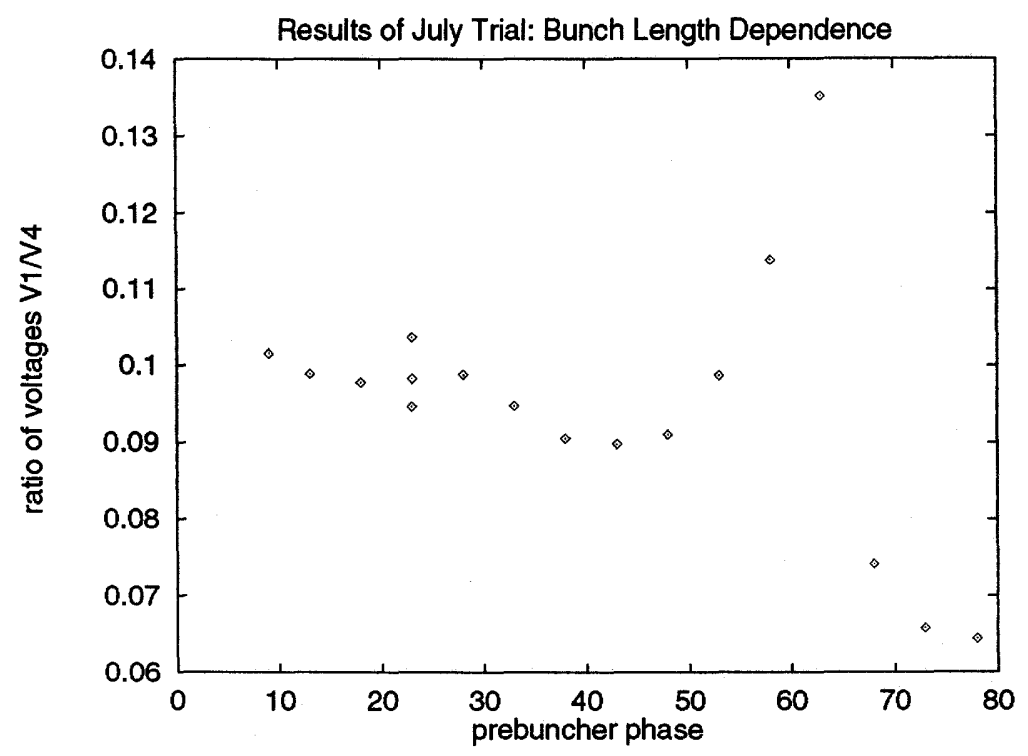

Figure 5.1: July Results: Experimental Dependence of Bunch Width on Prebuncher Phase

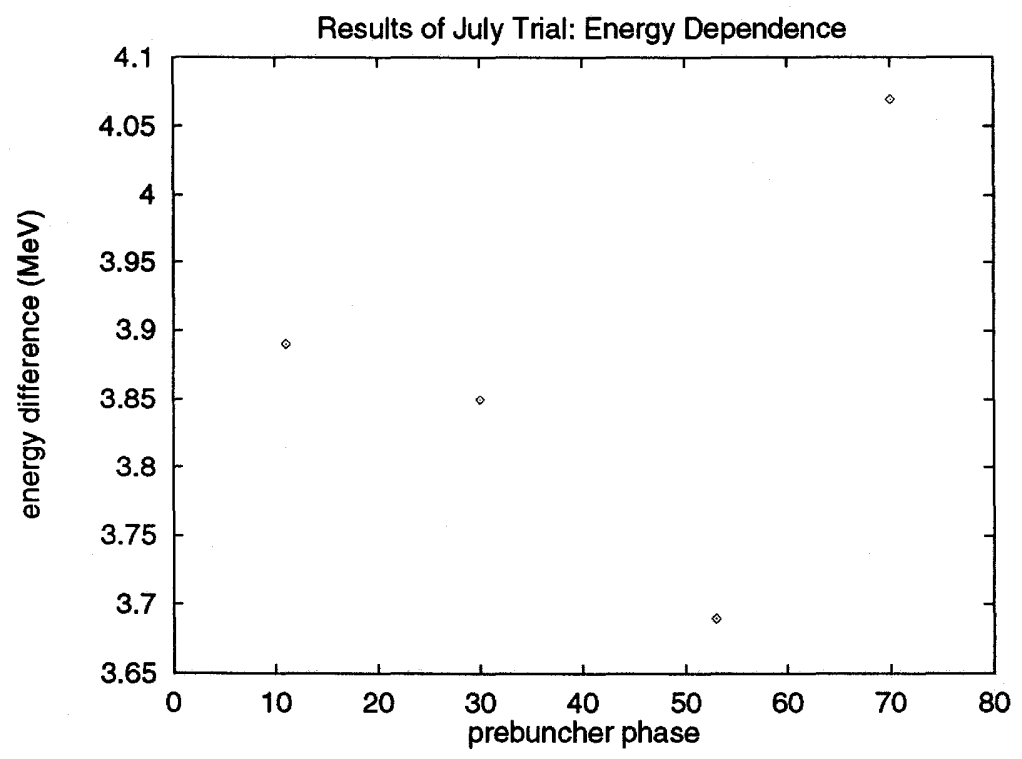

Figure 5.2: July Results: Experimental Dependence of Beam Energy on Prebuncher Phase 


\section{Appendix A}

\section{Filter Specifications}

This is a scan of the data sheet faxed to SAL from RF Microwave Ltd. [?]

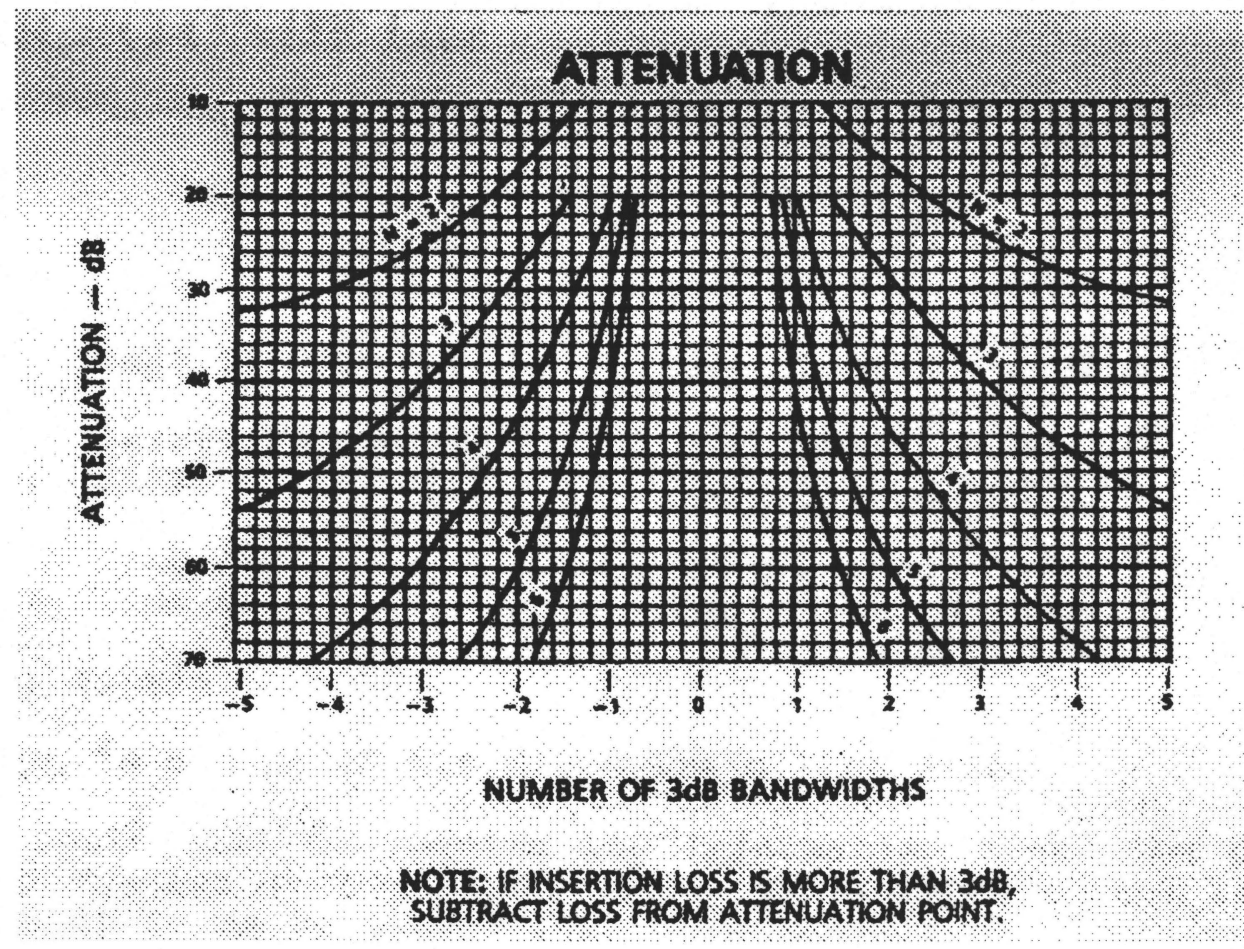

Figure A.1: Response Curve for Various Filters 


\title{
Appendix B
}

\section{Computer Programs}

\author{
Filtersim1.c
}

/* This program has a sine wave for input to a $2.856 \mathrm{GHz} \mathrm{BP}$ filter and */ /* outputs the simulation data to a file. The output plot is Figure 3.6.*/ \#include<stdio.h>

\#include<math.h>

\#define MAX 1000000

\#define $\mathrm{T} 0.5$

\#define PI 3.141592653591

main ()

\{

FILE *outfile, *fopen();

int limit,k;

double y[MAX] a $3, a 2, a 1, a 0, b 3, b 2, b 1, b 0, w, q$;

outfile = fopen ("sin1.dat", "w");

/* $\mathrm{w}$ is just the center frequency of the filter in $\mathrm{rad} / \mathrm{s}$ / $w=2 * P I / 350 ;$

/* the coefficents obtained from Octave for sampling period $\mathrm{T}=0.5 \mathrm{ps} * /$ a3 $=2.99520452884394 \mathrm{E}-9$;

$\mathrm{a} 2=-2.99529734348880 \mathrm{E}-9$;

$\mathrm{a} 1=-2.99501889955422 \mathrm{E}-9$;

$\mathrm{a} 0=2.99511182522139 \mathrm{E}-9$;

b3 = -3.999749249843368; 


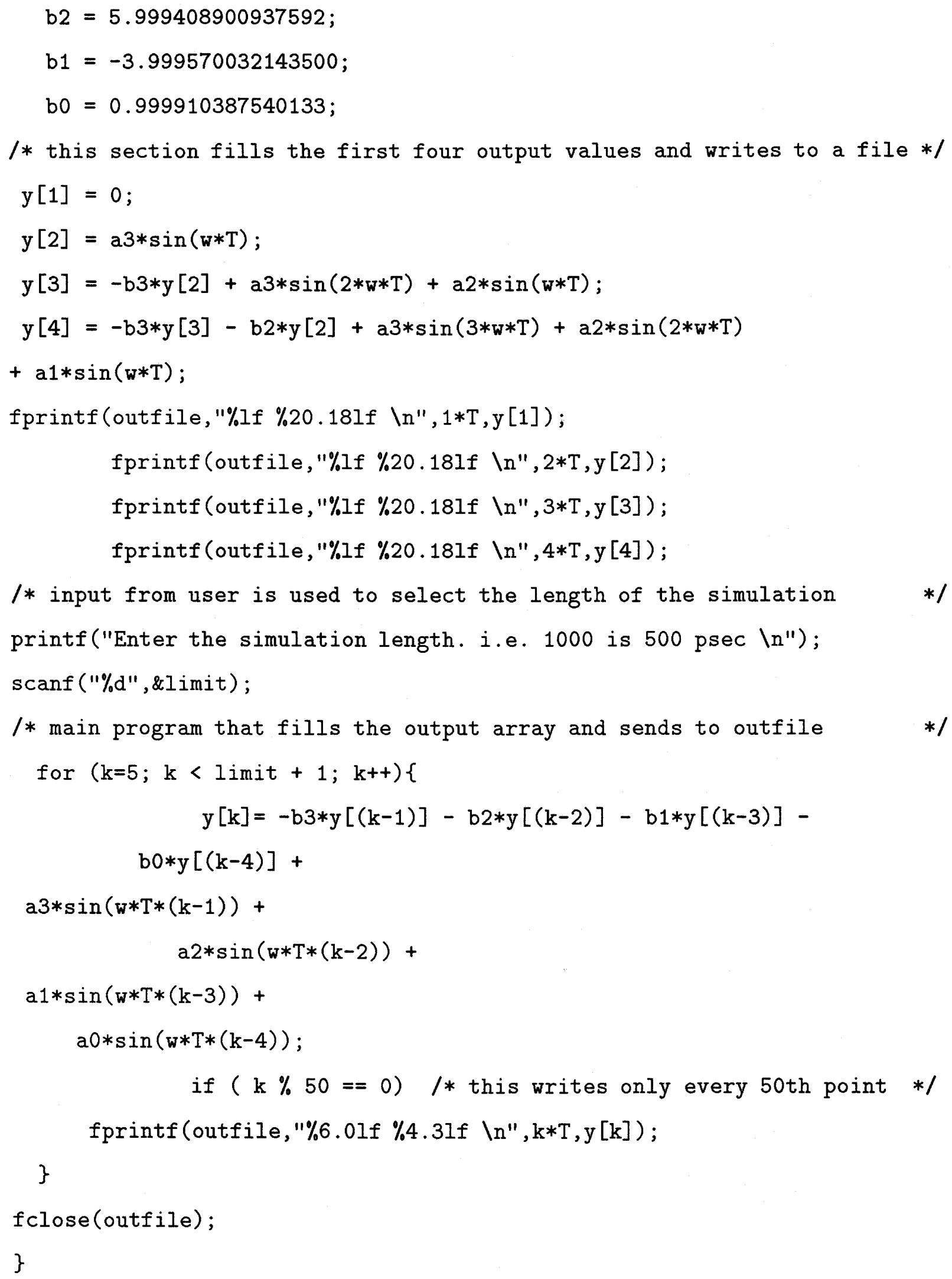




\title{
Filtersim4.c
}

/* This program has a sine wave for input to a $11.424 \mathrm{GHz}$ BP filter and */ /* outputs the simulation data to a file. The output plot is Figure 3.7.*/

\author{
\#include〈stdio.h〉 \\ \#include<math.h> \\ \#define MAX 1000000 \\ \#define $\mathrm{T} 0.1$ \\ \#define PI 3.141592653591
}

main ()

\{

FILE *outfile, *fopen();

int limit, $\mathrm{k}$;

double y[MAX] a $3, a 2, a 1, a 0, b 3, b 2, b 1, b 0, w$;

outfile = fopen ("sin4.dat", "w");

$/ * w$ is just the center frequency of the filter in rad/s

$$
\mathrm{w}=2 * \mathrm{PI} * 4 / 350 \text {; }
$$


/* the coefficents obtained from Dctave for sampling period $\mathrm{T}=0.1 \mathrm{ps} * /$

$$
\begin{aligned}
& \mathrm{a} 3=1.91694882190063 \mathrm{E}-9 ; \\
& \mathrm{a} 2=-1.91699012219715 \mathrm{E}-9 ; \\
& \mathrm{a} 1=-1.91686533312918 \mathrm{E}-9 ; \\
& \mathrm{a} 0=1.91690696649260 \mathrm{E}-9 ; \\
& \mathrm{b} 3=-3.999825180552219 ; \\
& \mathrm{b} 2=5.999578676849286 ; \\
& \mathrm{b} 1=-3.999681803027921 ; \\
& \mathrm{b} 0=0.999928309389544 ;
\end{aligned}
$$

/* this section fills the first four output values and writes to a file */

$$
\begin{aligned}
& \mathrm{y}[1]=0 ; \\
& y[2]=a 3 * \sin (w * T) ; \\
& y[3]=-b 3 * y[2]+a 3 * \sin (2 * w * T)+a 2 * \sin (w * T) ; \\
& y[4]=-b 3 * y[3]-b 2 * y[2]+a 3 * \sin (3 * w * T)+a 2 * \sin (2 * w * T) \\
& +a 1 * \sin (w * T)
\end{aligned}
$$

fprintf(outfile, "\%lf \%20.18lf In", $1 * \mathrm{~T}, \mathrm{y}[1]$ ); fprintf (outfile, "\%lf \%20.18lf $\backslash \mathrm{n} ", 2 * \mathrm{~T}, \mathrm{y}[2]$ ); fprintf(outfile, "\% lf \%20.18lf In",3*T,y[3]); fprintf(outfile, "\% If \%20.18lf $\backslash \mathrm{n} ", 4 * \mathrm{~T}, \mathrm{y}[4]$ );

/* input from user is used to select the length of the simulation

printf("Enter the simulation length. i.e. 1000 is $100 \mathrm{psec} \backslash \mathrm{n} "$ ); 


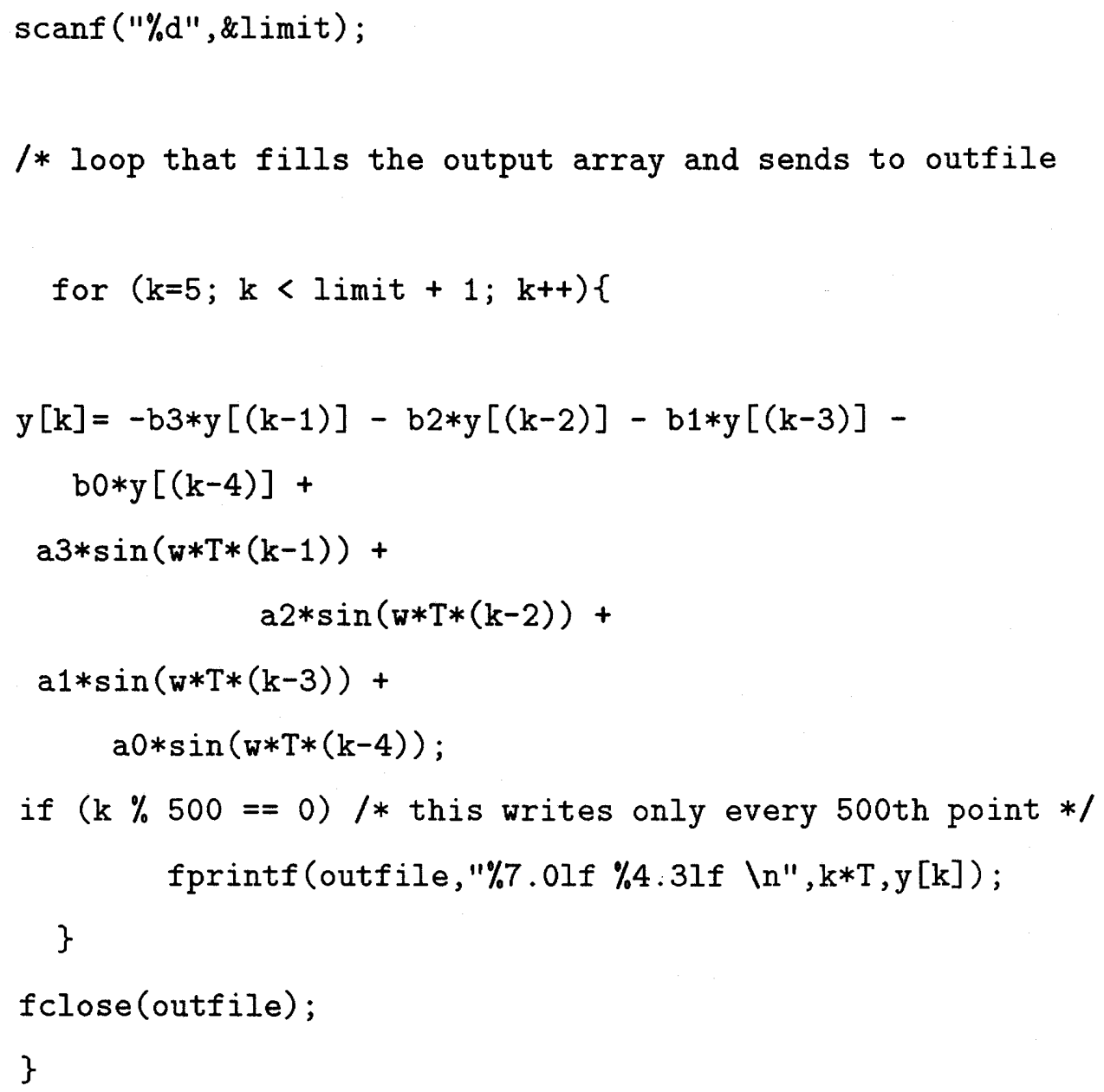




\section{Filtersim1g.c}

/* This program has a Gaussian wavetrain (Figure 2.5) for input of a */ /*2.856 GHz BP and outputs the simulated data to a file. The output */ /* plot is Figure 3.8. */

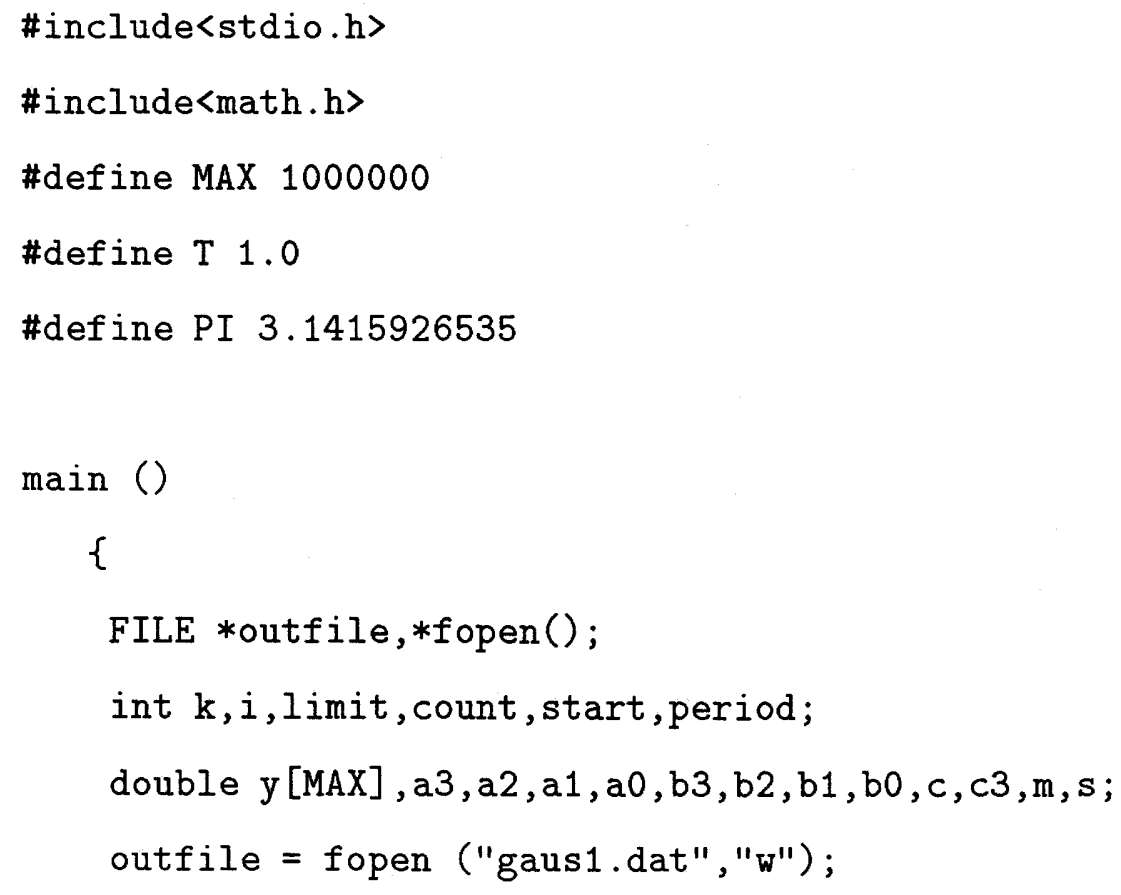

/* These are the initial conditions set by the user for the simulation. 
/* They include the length of simulation, the sigma of the Gaussian

/* pulse, the period of the wavetrain and the position of the peak of */

/* the first doublet (mean).

printf("Enter the value of $\mathbf{k}(1000$ is $1000 \mathrm{psec}) . \ln ")$;

$\operatorname{scanf}(" \% d ", \& l i m i t)$;

printf("Enter the value of sigma ( 8.0 is 8 psec). $\ln ")$;

$\operatorname{scanf}(" \% 1 f ", 8 s)$;

printf("Enter the value of period (350 is 350 psec). $\left(\mathrm{n}^{\prime \prime}\right)$;

scanf ("\%d", \&period);

printf("Enter the value of the mean ( 7.0 equals $7 *$ sigma). $\backslash n ")$;

$\operatorname{scanf}(" \% 1 f ", \& m)$;

/* initial conditions for $2.856 \mathrm{GHz}$

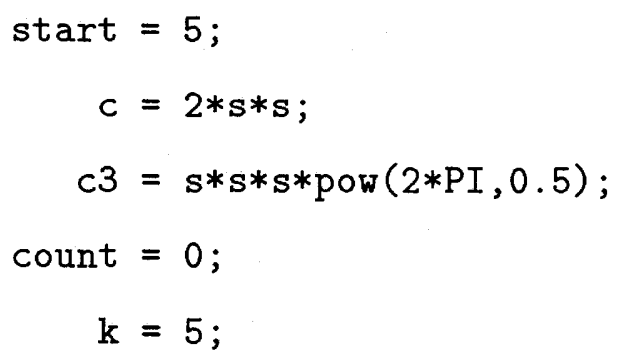

/* the coefficents obtained from Octave for sampling period $\mathrm{T}=1.0 \mathrm{ps} \quad * /$

$$
\begin{aligned}
& \quad \mathrm{a} 3=1.19799712372526 \mathrm{E}-8 ; \\
& \mathrm{a} 2=-1.19806893295049 \mathrm{E}-8 ; \\
& \mathrm{a} 1=-1.19785372731940 \mathrm{E}-8 ; \\
& \mathrm{a} 0=1.19792545882902 \mathrm{E}-8 ; \\
& \text { b3 }=-3.999176259747399 ;
\end{aligned}
$$


$\mathrm{b} 2=5.998173464205498 ;$

$\mathrm{b} 1=-3.998817883723882 ;$

$\mathrm{b} 0=0.999820783110653 ;$

/* this section fills the first four output values and writes to a file */

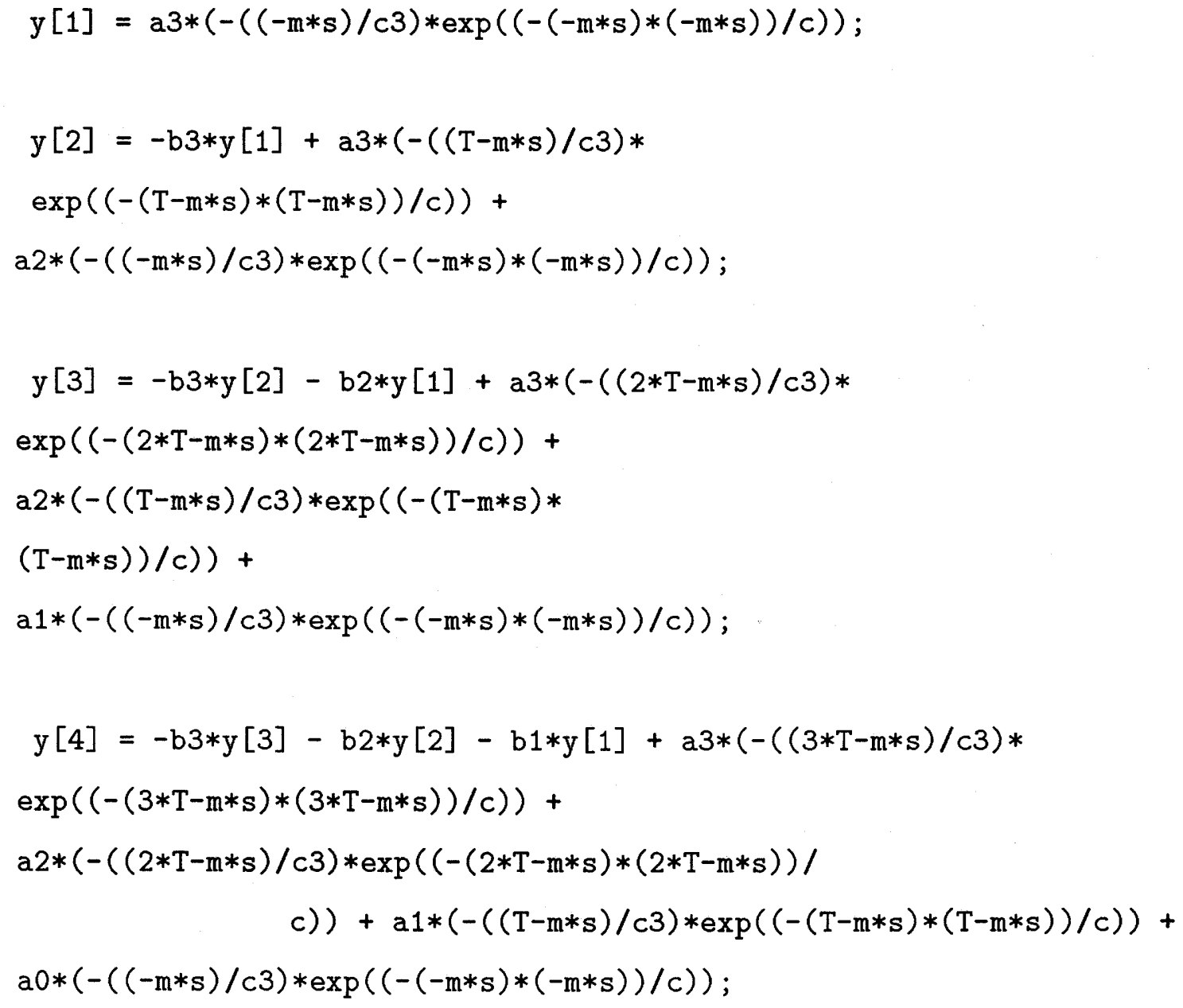


/* The WHILE loop continues the FOR loop for the simulation length. The */ /* FOR loop continues the process of filling the output array for each */ /* successive wavetrain period. The $\mathrm{k}$ values repesent the sampling points $* /$ /* for the length of the simulation and $i$ resets for each successive */ /* wavetrain period. */

while ( $k<$ limit $)\{$

for $(i=$ start $; i<\operatorname{period}+1 ; i++)\{$

$\mathrm{y}[\mathrm{k}]=-\mathrm{b} 3 * \mathrm{y}[(\mathrm{k}-1)]-\mathrm{b} 2 * \mathrm{y}[(\mathrm{k}-2)]-\mathrm{b} 1 * \mathrm{y}[(\mathrm{k}-3)]-$

$\mathrm{b} 0 * \mathrm{y}[(\mathrm{k}-4)]+$

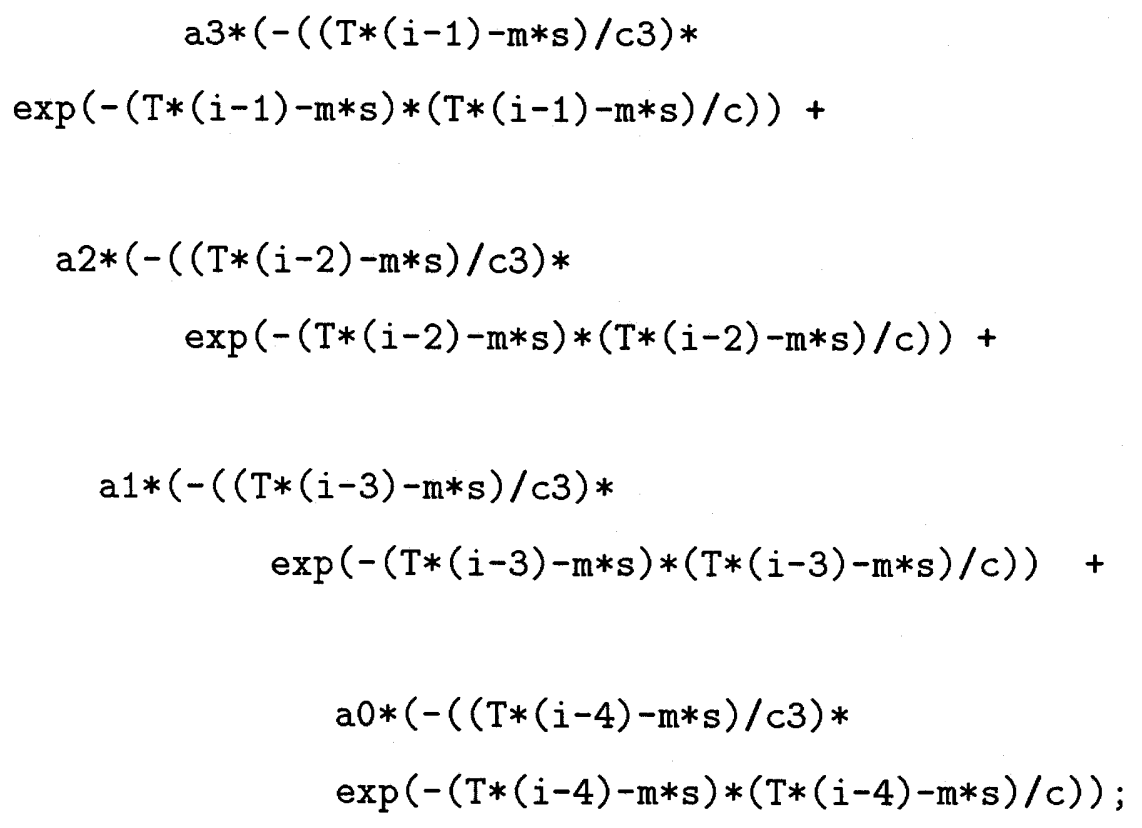




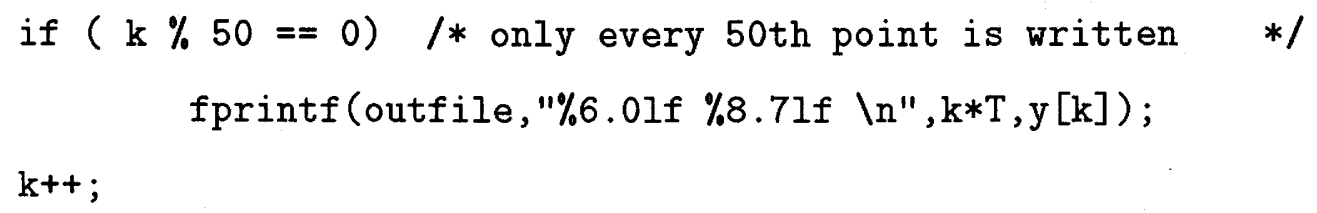




\title{
Filtersim4g.c
}

/* This program has a differentiated Gaussian wavetrain (Figure 2.6) for */ /* input of a $11.424 \mathrm{GHz} \mathrm{BPF}$ and outputs the simulated data to a file. The*/ /* output plot is Figure 3.9 .

\author{
\#include<stdio.h> \\ \#include<math.h> \\ \#define MAX 1000000 \\ \#define $\mathrm{T} 1.0$ \\ \#define PI 3.1415926535
}

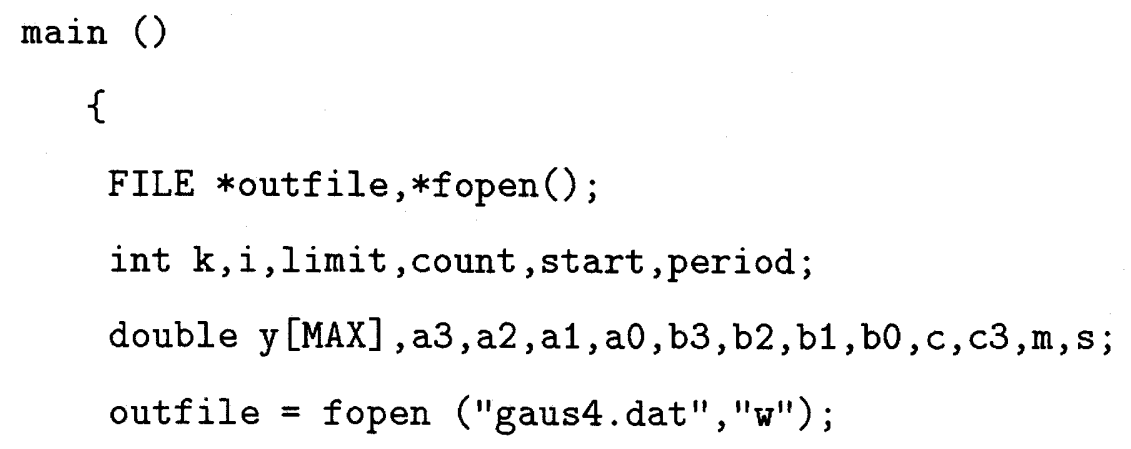

/* These are the initial conditions set by the user for the simulation. */ /* They include the length of simulation, the sigma of the Gaussian */ 
/* pulse, the period of the wavetrain and the position of the peak of */ /* the first doublet (mean). */

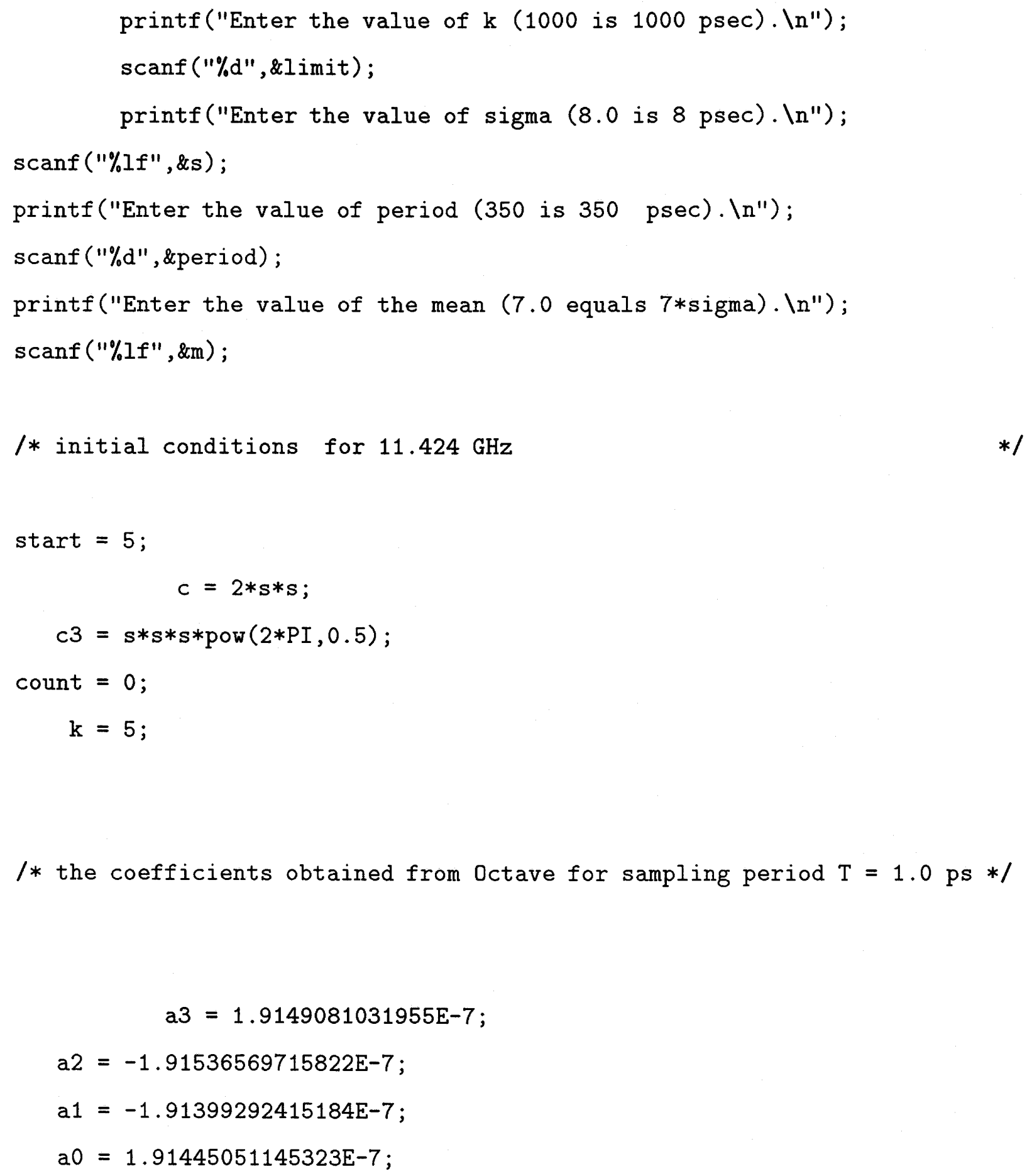


b3 $=-3.988976490760761$;

$\mathrm{b} 2=5.977266556236755$;

$\mathrm{b} 1=-3.987546834871388$;

bo $=0.999283325130680$;

/* this section fills the first four output values and writes to a file */

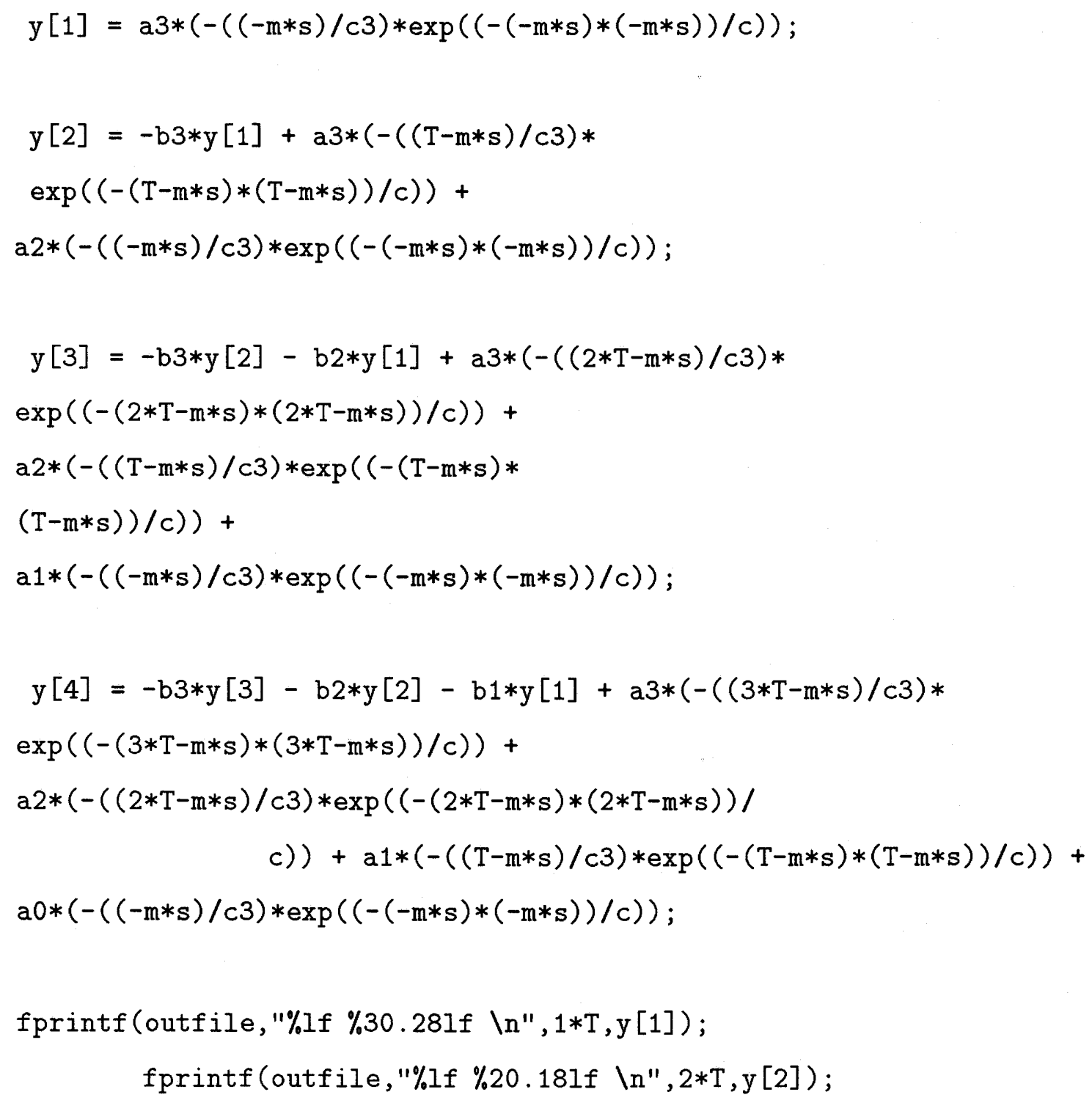


fprintf(outfile, "\%lf $\% 20.181 f \backslash \mathrm{n} ", 3 * \mathrm{~T}, \mathrm{y}[3]$ );

fprintf(outfile, "\%lf \%20.18lf $\backslash n ", 4 * T, y[4]$ );

I* The WHILE loop continues the FOR loop for the simulation length. The */ /* FOR loop continues the process of filling the output array for each */ /* successive wavetrain period. The $\mathrm{k}$ values repesent the sampling points $* /$ /* for the length of the simulation and $i$ resets for each successive */ /* wavetrain period. */

while (k < limit ) \{

for $(i=$ start; $i<$ period $+1 ; i++)\{$

$\mathrm{y}[\mathrm{k}]=-\mathrm{b} 3 * \mathrm{y}[(\mathrm{k}-1)]-\mathrm{b} 2 * \mathrm{y}[(\mathrm{k}-2)]-\mathrm{b} 1 * \mathrm{y}[(\mathrm{k}-3)]-$

$\mathrm{b} 0 * \mathrm{y}[(\mathrm{k}-4)]+$

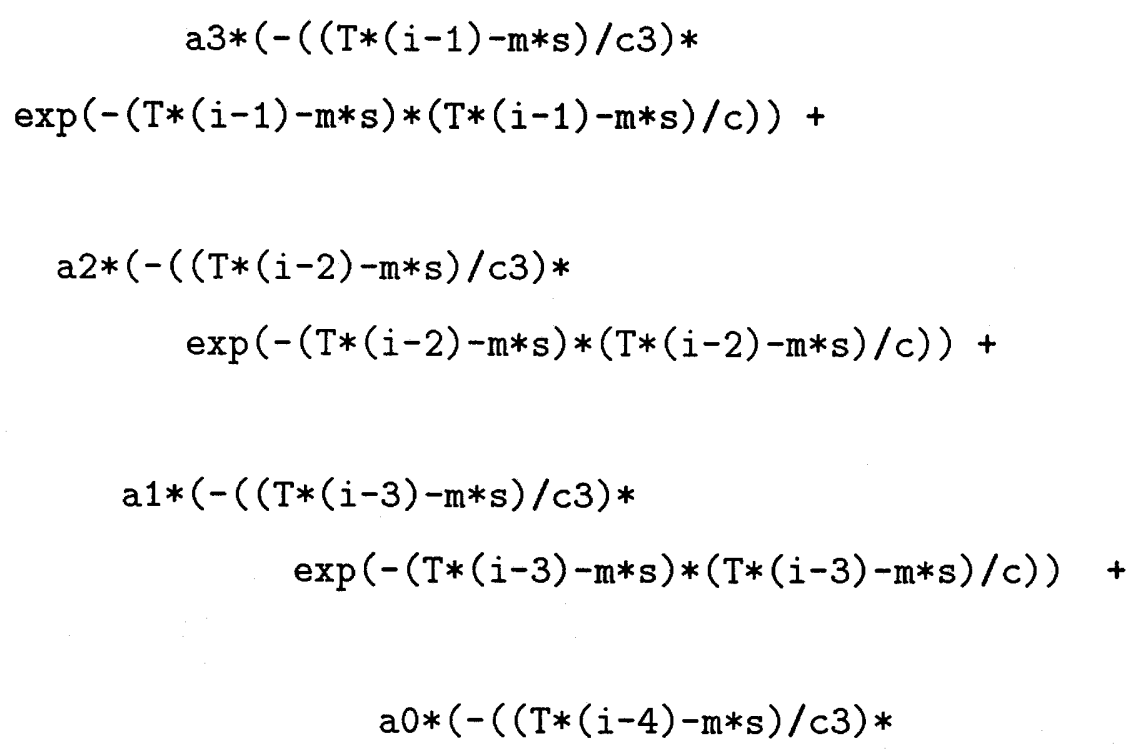




$$
\exp (-(T *(i-4)-m * s) *(T *(i-4)-m * s) / c))
$$

if $(\mathrm{k} \% 50==0) \quad / *$ only every 50 th point is written */ fprintf (outfile, "\%6.0lf \%8.7lf $\backslash \mathrm{n} ", \mathrm{k} * \mathrm{~T}, \mathrm{y}[\mathrm{k}]$ );

$\mathrm{k++}$;

\}

count++;

$\mathrm{k}=$ count $*$ period +1

start $=1 ;$

\}

fclose(outfile);

\} 


\section{Appendix C}

\section{Bench Test Results}

The results from the bench tests for various buttons are included below. All horizontal scales are $200 \mathrm{~ns} /$ div. The vertical scales vary.

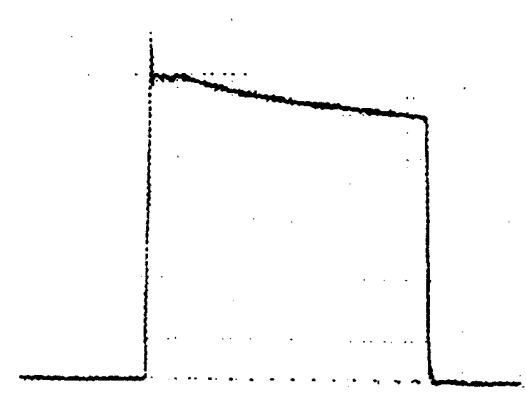

Figure C.1: $\quad$ V.S. $=4 \mathrm{~mW} / \mathrm{div} ;$ TSO Raw Output

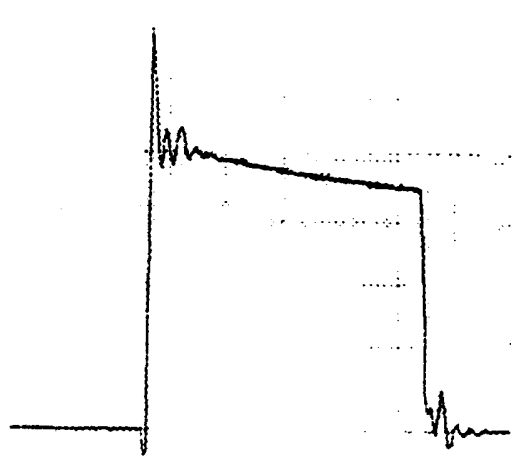

Figure C.3: V.S. $=3 \mathrm{~mW} / \mathrm{div} ; \mathrm{TSO}$ Output at $2856 \mathrm{MHz}$

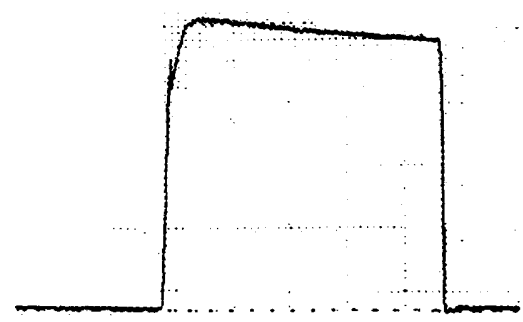

Figure C.2: V.S. $=200 \mu \mathrm{W} /$ div; Raw Beam Pipe Output

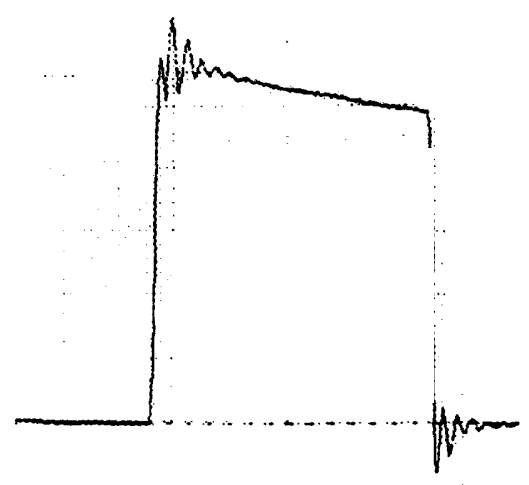

Figure C.4: V.S. $=60 \mu \mathrm{W} /$ div; Beam Pipe Output at $2856 \mathrm{MHz}$ 


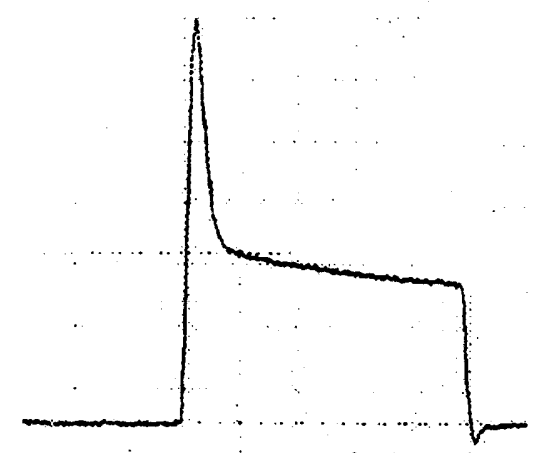

Figure C.5: V.S. $=30 \mu \mathrm{W} /$ div; TSO Output at $11424 \mathrm{MHz}$

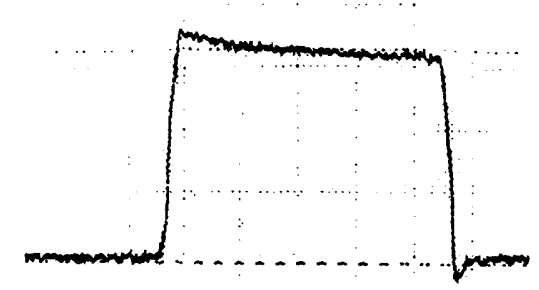

Figure C.7: V.S. $=5 \mu \mathrm{W} /$ div; Raw Output for $0.5 \mathrm{~cm}$ Diameter Button

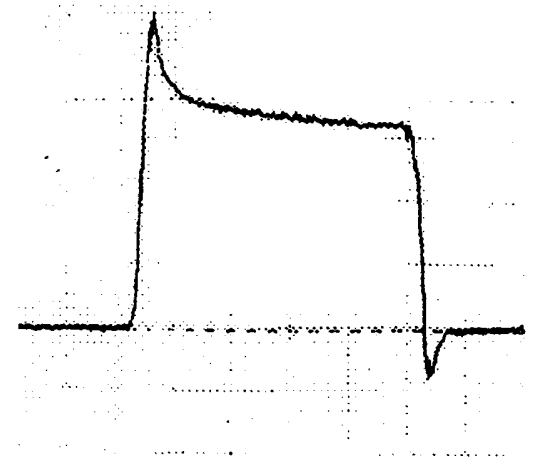

Figure C.6: V.S. $=7 \mu \mathrm{W} /$ div; Beam Pipe Output at $11424 \mathrm{MHz}$

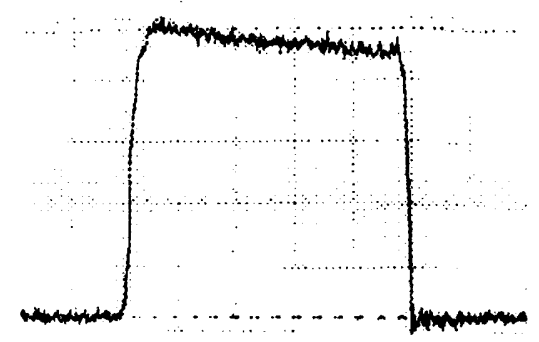

Figure C.8: V.S. $=8 \mu \mathrm{W} /$ div; Raw Output for $1 \mathrm{~cm}$ Diameter Button 


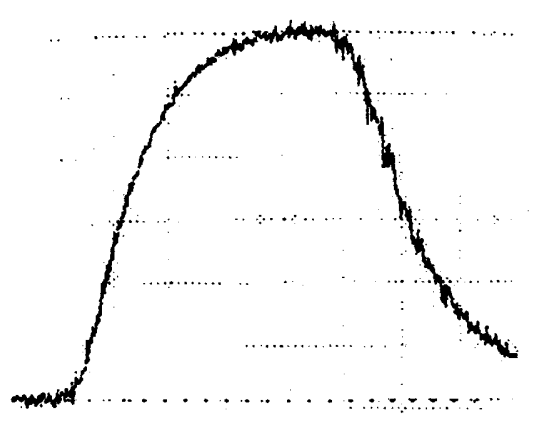

Figure C.9: V.S. $=600 \mathrm{nW} /$ div; 2856 $\mathrm{MHz}$ Output for $0.5 \mathrm{~cm}$ Diameter Button

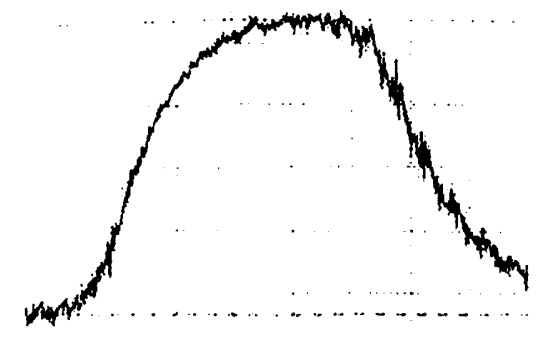

Figure C.11: V.S. $=500 \mathrm{nW} /$ div $; 11424$ $\mathrm{MHz}$ Output for $0.5 \mathrm{~cm}$ Diameter Button

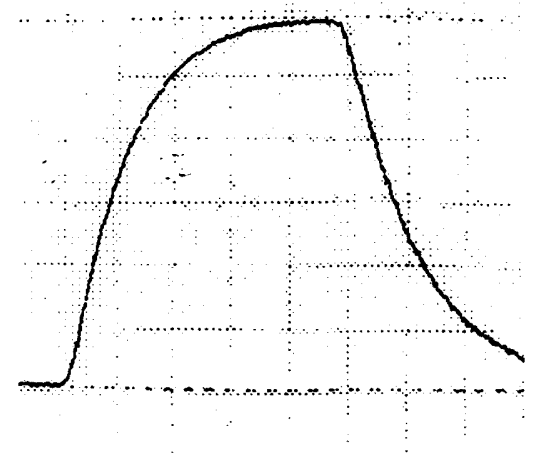

Figure C.10: V.S. $=2.5 \mu \mathrm{W} /$ div $; 2856$ $\mathrm{MHz}$ Output for $1 \mathrm{~cm}$ Diameter Button

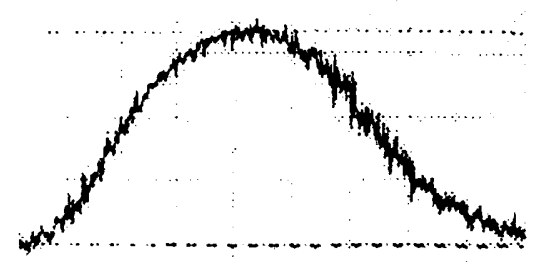

Figure C.12: V.S. $=450 \mathrm{nW} /$ div; 11424 $\mathrm{MHz}$ Output for $1 \mathrm{~cm}$ Diameter Button 


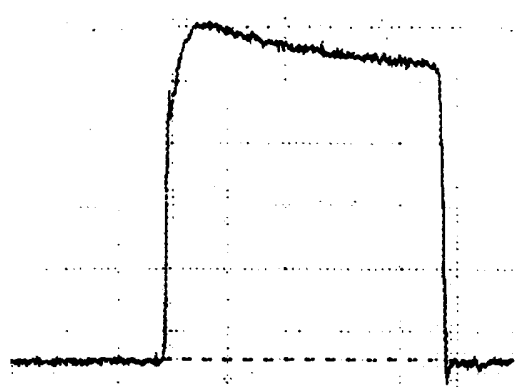

Figure C.13: V.S. $=12 \mu \mathrm{W} /$ div; Raw Output for $2.2 \mathrm{~cm}$ Diameter Button

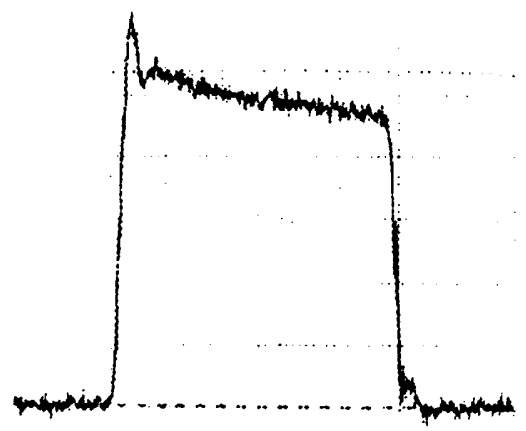

Figure C.15: V.S. $=6 \mu \mathrm{W} / \mathrm{div} ; 2856$ $\mathrm{MHz}$ Output for $2.2 \mathrm{~cm}$ Diameter Button

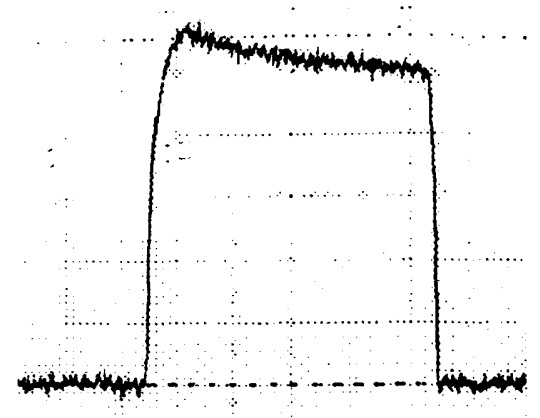

Figure C.14: V.S. $=6 \mu \mathrm{W} /$ div; Raw Output for $2 \mathrm{~cm}$ Square Button

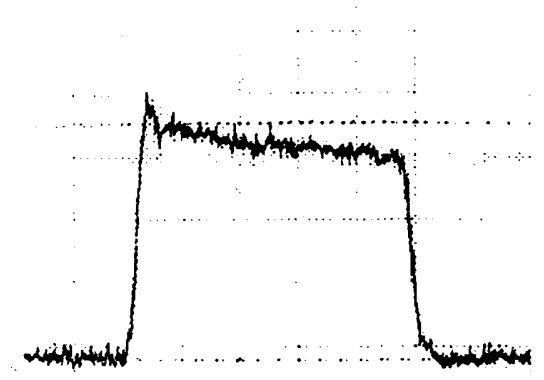

Figure C.16: V.S. $=6 \mu \mathrm{W} /$ div; 2856 $\mathrm{MHz}$ Output for $2 \mathrm{~cm}$ Square Button 


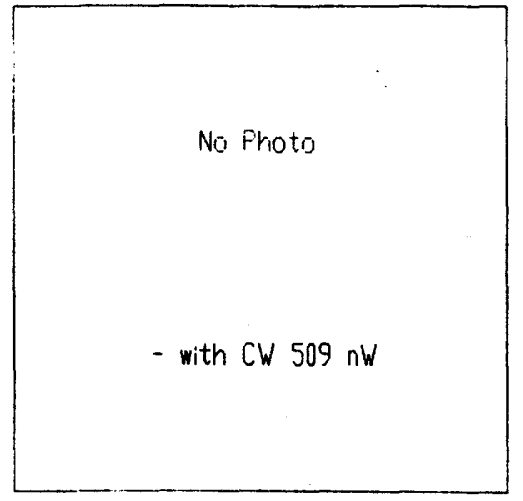

Figure C.17: Continuous Wave Output for $11424 \mathrm{MHz} 2.2 \mathrm{~cm}$ Diameter Button

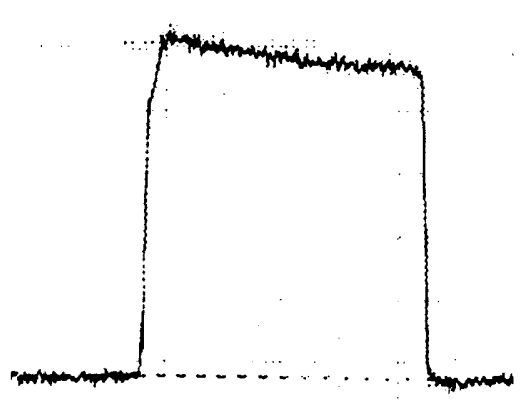

Figure C.19: V.S. $=8 \mu \mathrm{W} /$ div; Raw Output for $1 \mathrm{~cm}$ Square Button

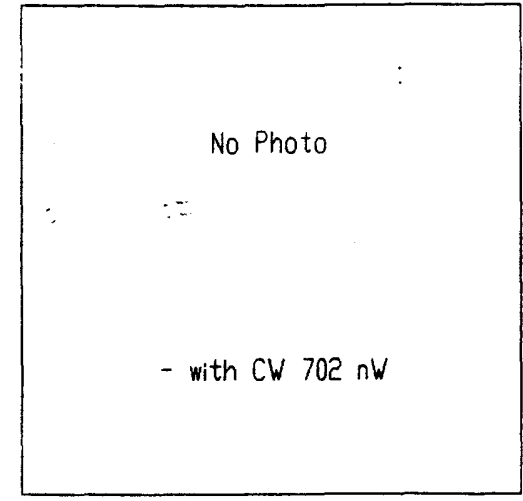

Figure C.18: Continuous Wave Output for $11424 \mathrm{MHZ} 2 \mathrm{~cm}$ Square Button

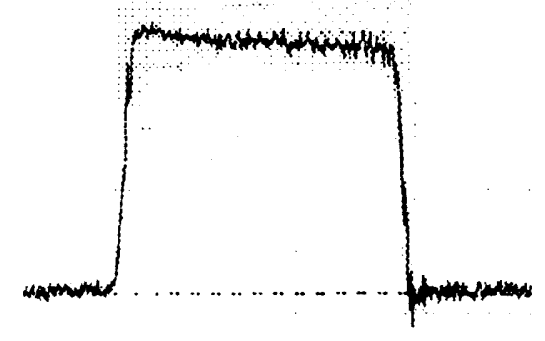

Figure C.20: V.S. $=6 \mu \mathrm{W} / \mathrm{div} ;$ Raw Output for $0.5 \mathrm{~cm}$ Square Button 


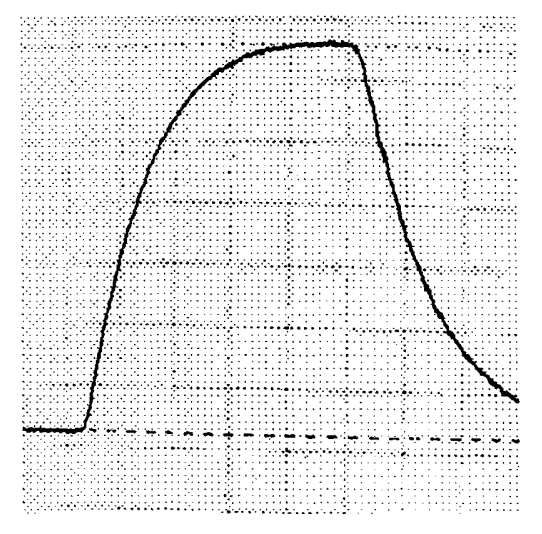

Figure C.1: V.S. $=2.5 \mu \mathrm{W} /$ div; 2856 $\mathrm{MHz}$ Output for $1 \mathrm{~cm}$ Square Button

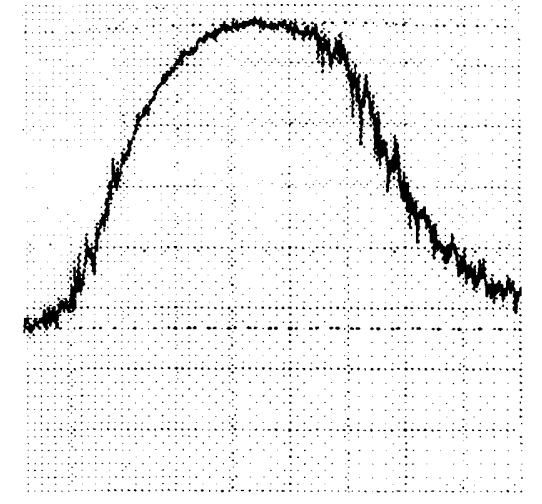

Figure C.3: V.S. $=450 \mathrm{nW} /$ div; 11424 $\mathrm{MHz}$ Output for $1 \mathrm{~cm}$ Square Button

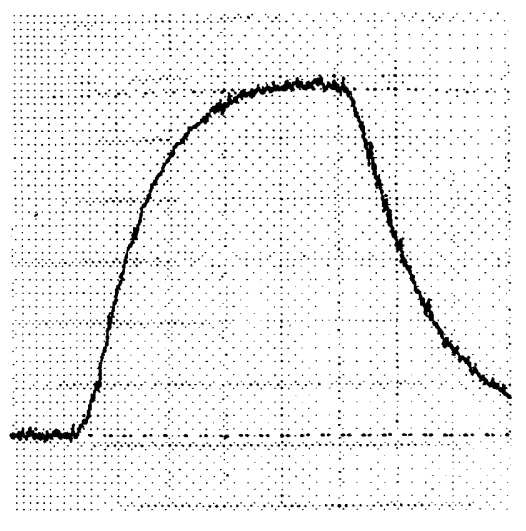

Figure C.2: V.S. $=800 \mathrm{nW} /$ div; 2856 $\mathrm{MHz}$ Output for $0.5 \mathrm{~cm}$ Square Button

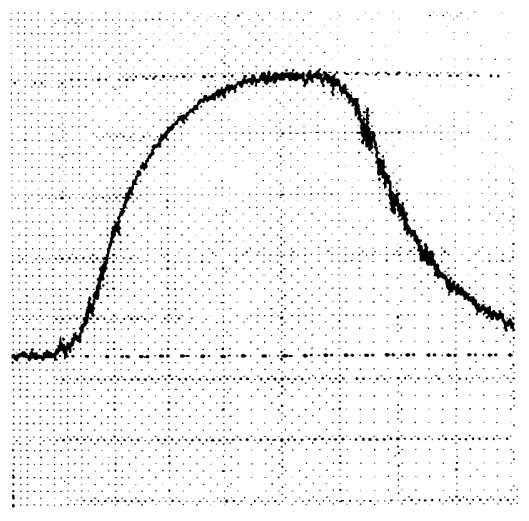

Figure C.4: V.S. $=800 \mathrm{nW} /$ div; 11424 $\mathrm{MHz}$ Output for $0.5 \mathrm{~cm}$ Square Button 


\section{Appendix D}

\section{April Results with Peak Power Meter}

The results of the April run are illustrated below. The output of the filters measured by the Peak Power Meter and were stored on the NeXT computer as digitized data points. The prebuncher phase was varied and the output was subtracted from the orginally stored data points.

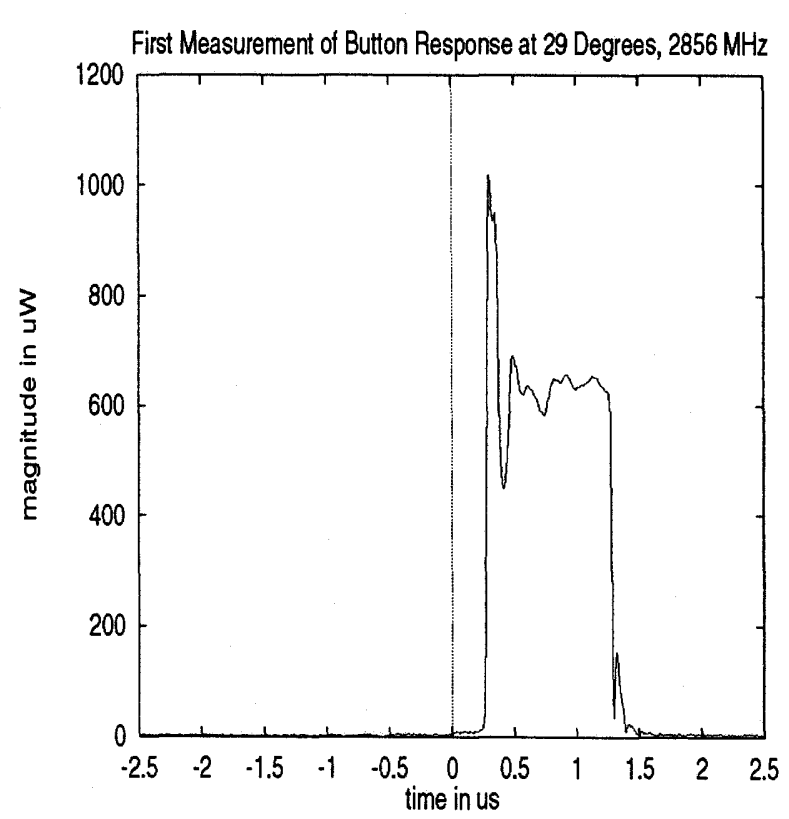

Figure D.1: $2856 \mathrm{MHz}$ : Prebuncher Phase $29^{\circ}$

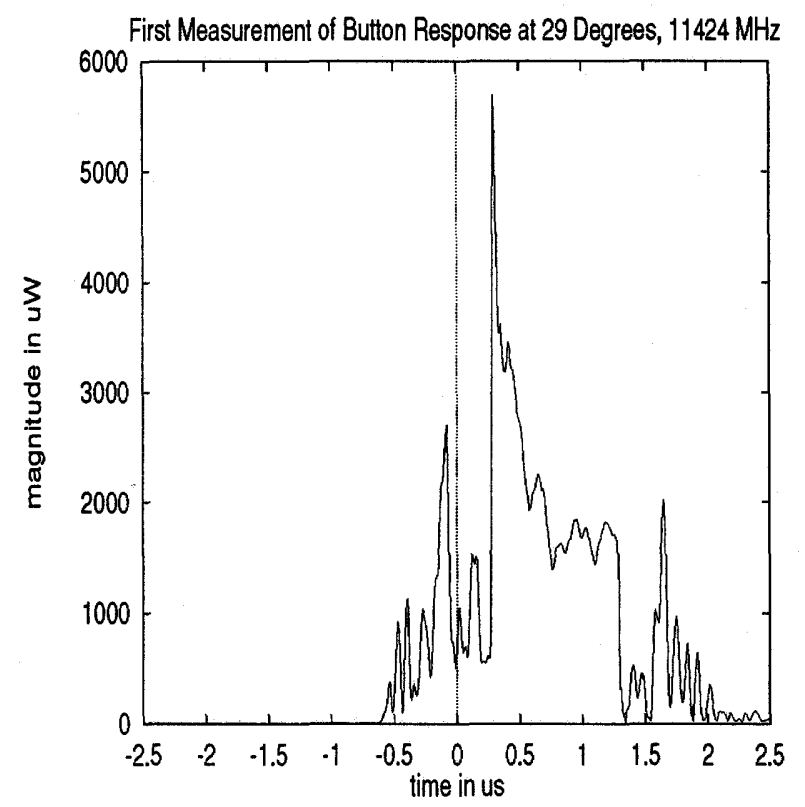

Figure D.2: $11424 \mathrm{MHz}$ : Prebuncher Phase $29^{\circ}$ 


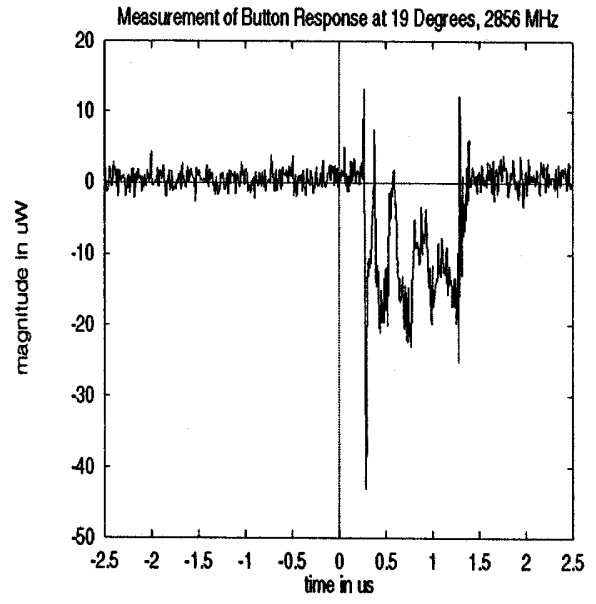

Figure D.3: $2856 \mathrm{MHz}$ : Prebuncher Phase $19^{\circ}$

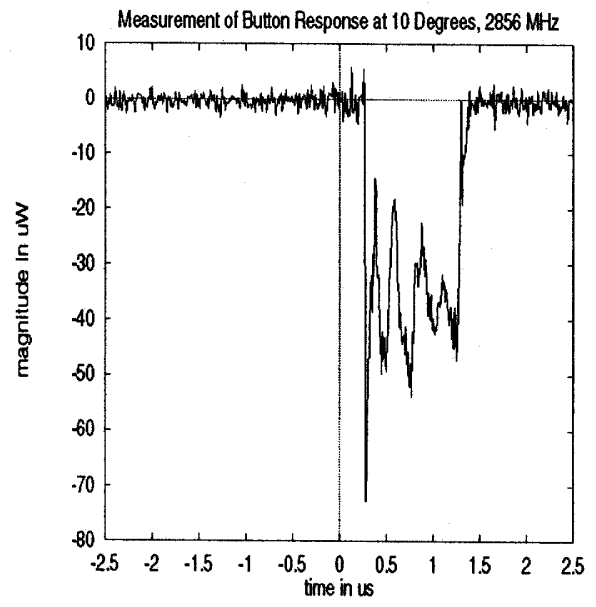

Figure D.5: $2856 \mathrm{MHz}$ : Prebuncher Phase $10^{\circ}$

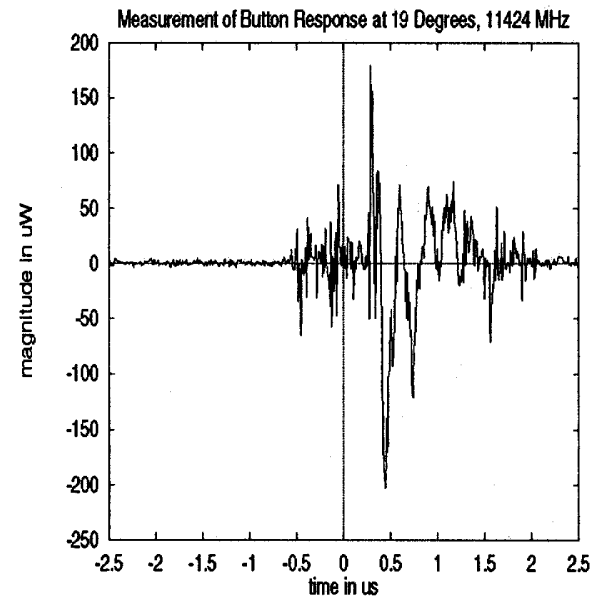

Figure D.4: $11424 \mathrm{MHz}$ : Prebuncher Phase $19^{\circ}$

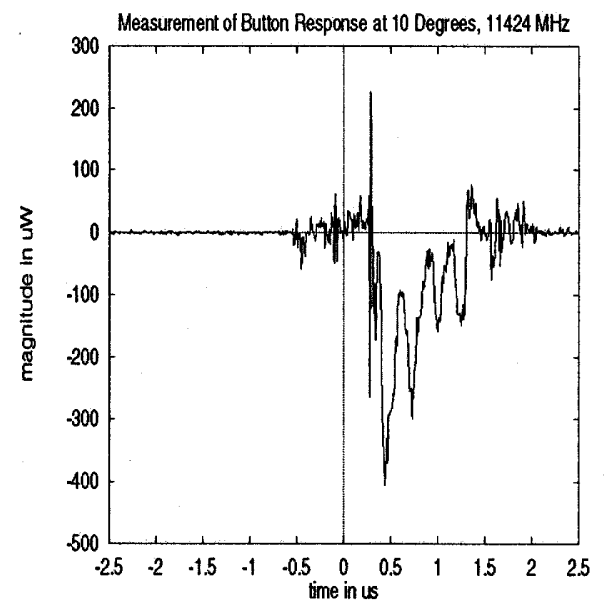

Figure D.6: $11424 \mathrm{MHz}$ : Prebuncher Phase $10^{\circ}$ 


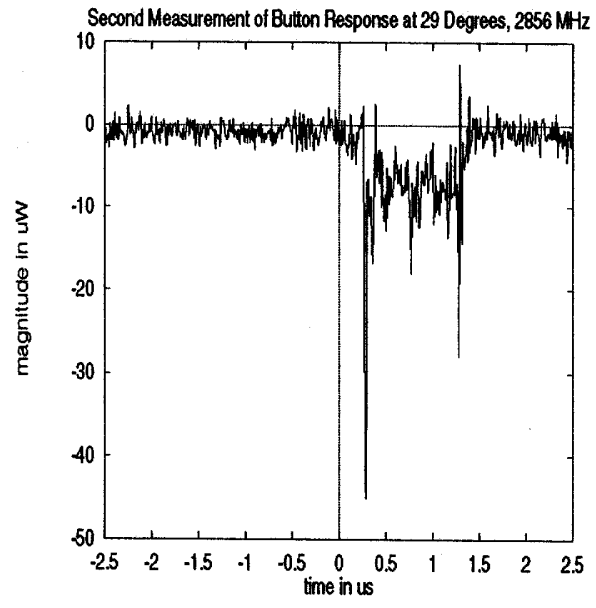

Figure D.7: $2856 \mathrm{MHz}$ : Prebuncher Phase $29^{\circ}$

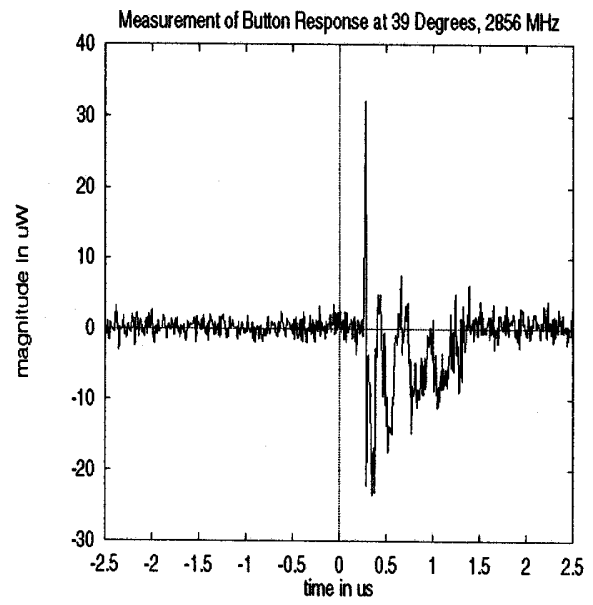

Figure D.9: $2856 \mathrm{MHz}$ : Prebuncher Phase $39^{\circ}$

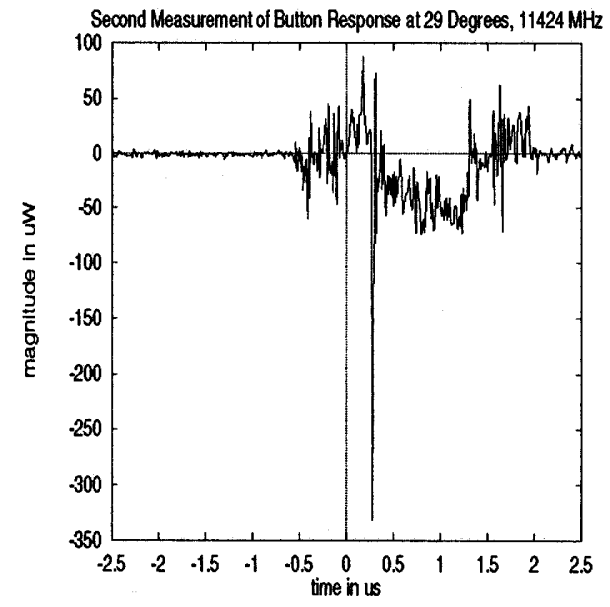

Figure D.8: $11424 \mathrm{MHz}$ : Prebuncher Phase $29^{\circ}$

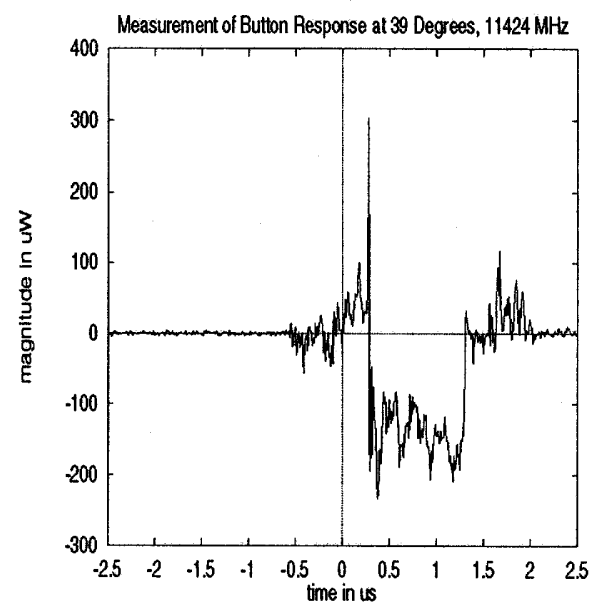

Figure D.10: $11424 \mathrm{MHz}$ : Prebuncher Phase $39^{\circ}$ 


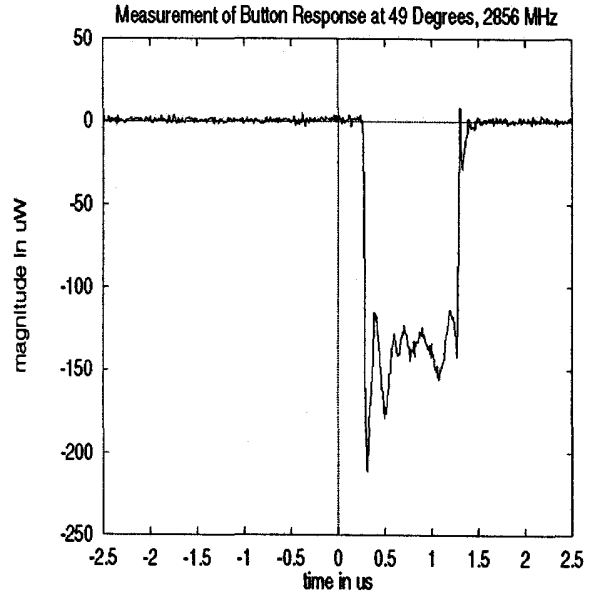

Figure D.11: $2856 \mathrm{MHz}$ : Prebuncher Phase $49^{\circ}$

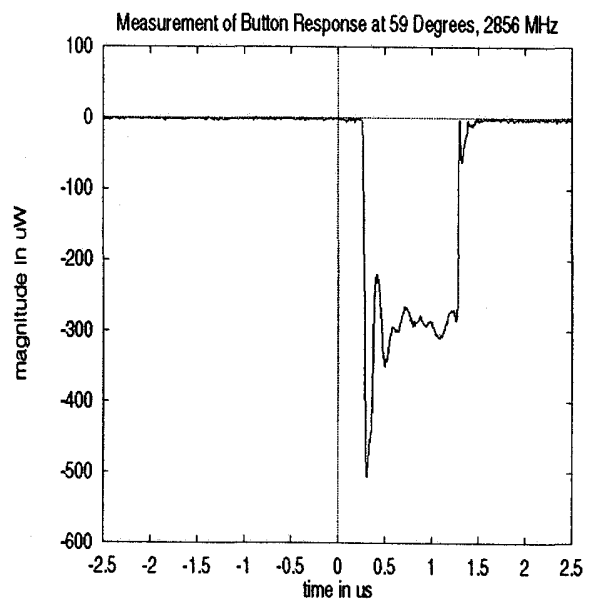

Figure D.13: $2856 \mathrm{MHz}$ : Prebuncher Phase $59^{\circ}$

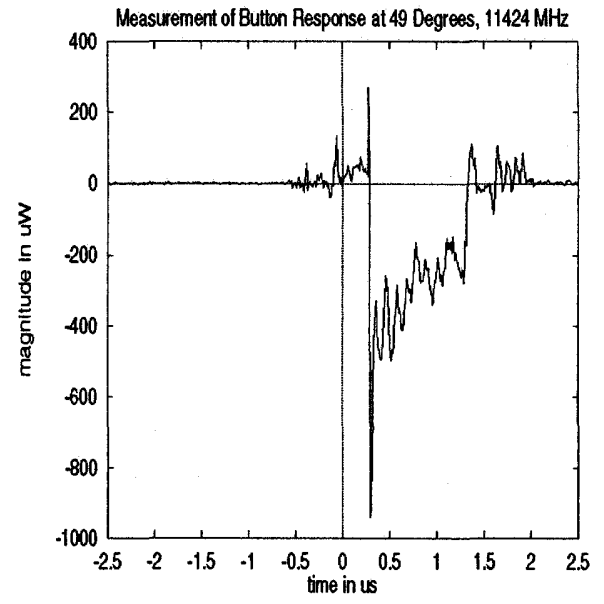

Figure D.12: $11424 \mathrm{MHz}$ : Prebuncher Phase $49^{\circ}$

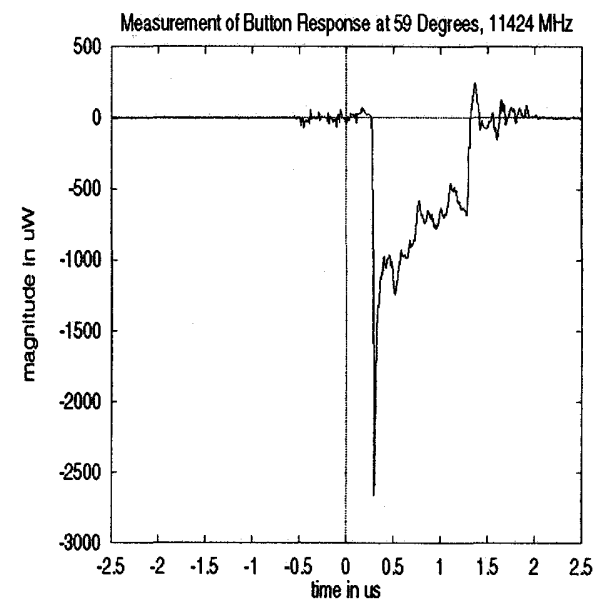

Figure D.14: $11424 \mathrm{MHz}$ : Prebuncher Phase $59^{\circ}$ 


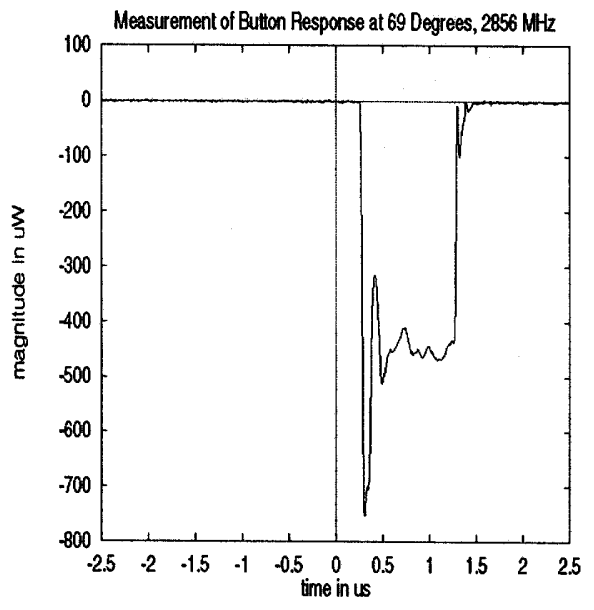

Figure D.15: $2856 \mathrm{MHz}$ : Prebuncher Phase $69^{\circ}$

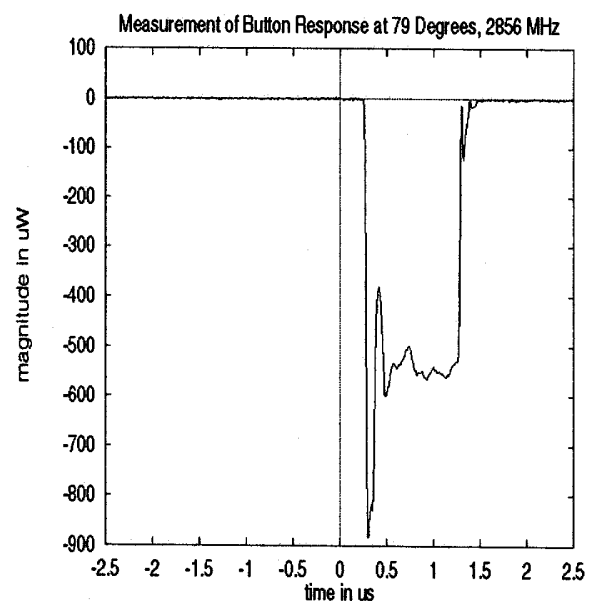

Figure D.17: $2856 \mathrm{MHz}$ : Prebuncher Phase $79^{\circ}$

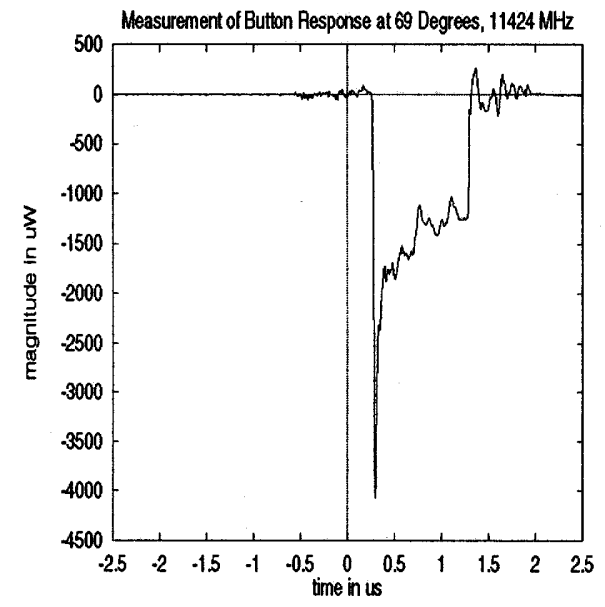

Figure D.16: $11424 \mathrm{MHz}$ : Prebuncher Phase $69^{\circ}$

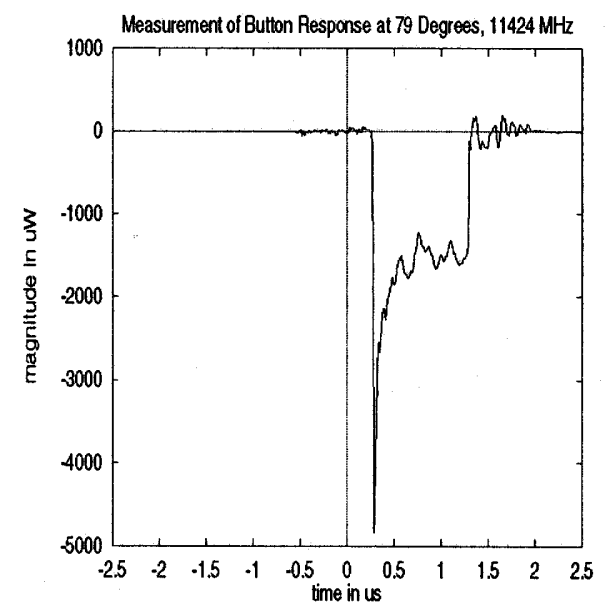

Figure D.18: $11424 \mathrm{MHz}$ : Prebuncher Phase $79^{\circ}$ 


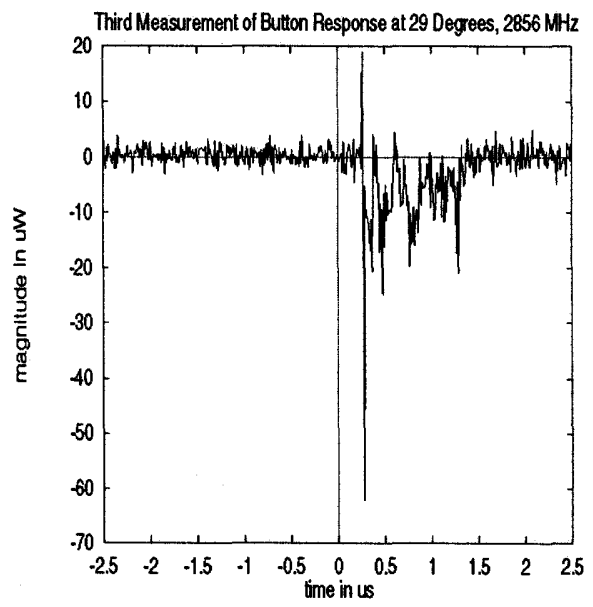

Figure D.19: $2856 \mathrm{MHz}$ : Prebuncher Phase $29^{\circ}$

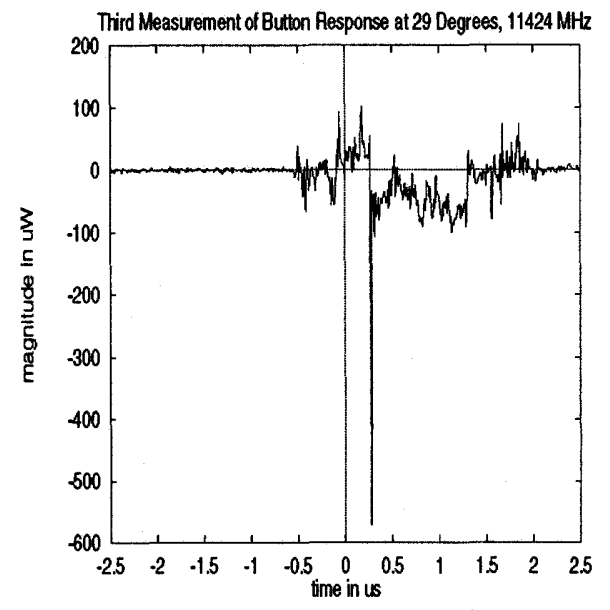

Figure D.20: $11424 \mathrm{MHz}$ : Prebuncher Phase $29^{\circ}$ 


\section{Appendix E}

\section{April Results with Crystal Detectors}

The scope traces as saved by the NeXT computer are illustrated below. The raw output of the crystal detectors are given first in Figures D.1 and D.2. Then the noise is subtracted from the pulse and illustrated in Figures D.3 and D.4. The other Figures have the prebuncher phase varied and the output recorded.

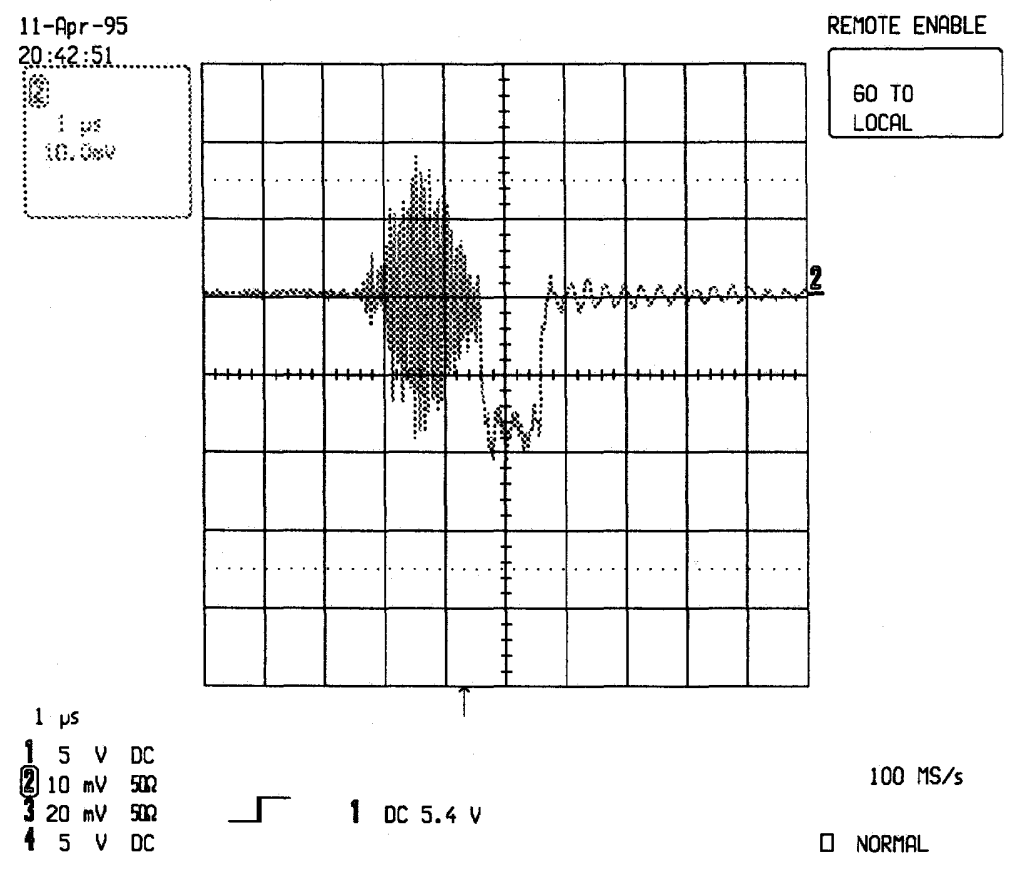

Figure E.1: Raw $2856 \mathrm{MHz}$ Signal 


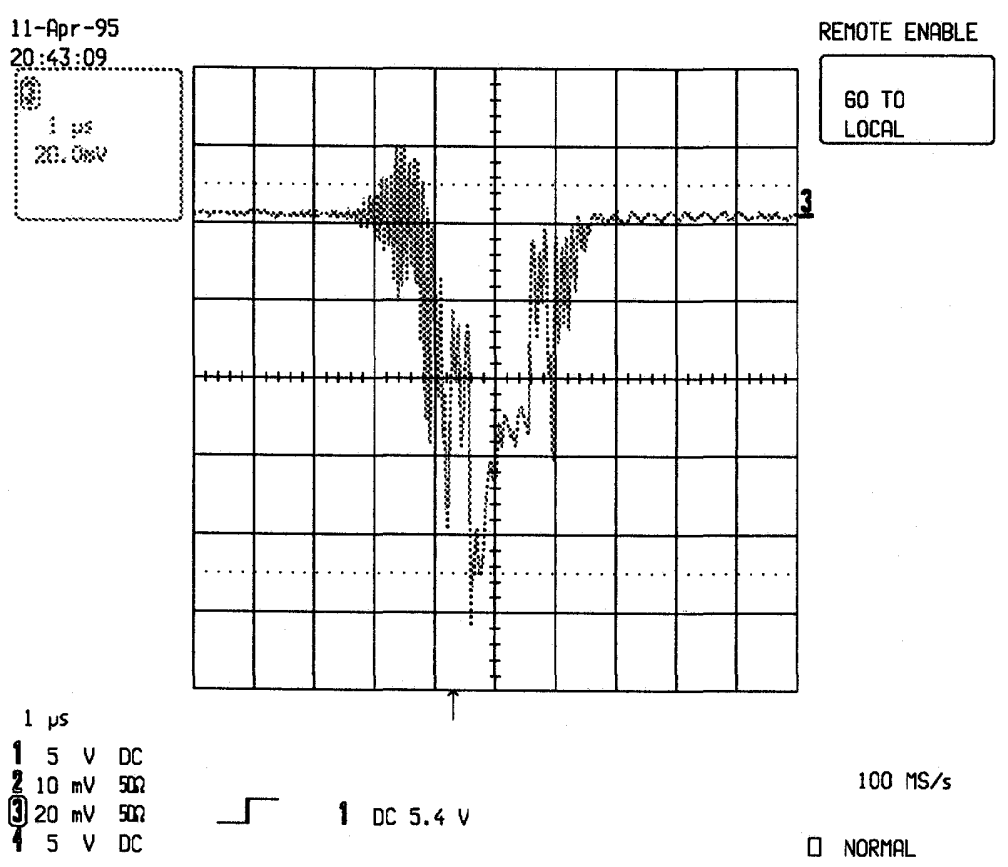

Figure E.2: Raw $11424 \mathrm{MHz}$ Signal

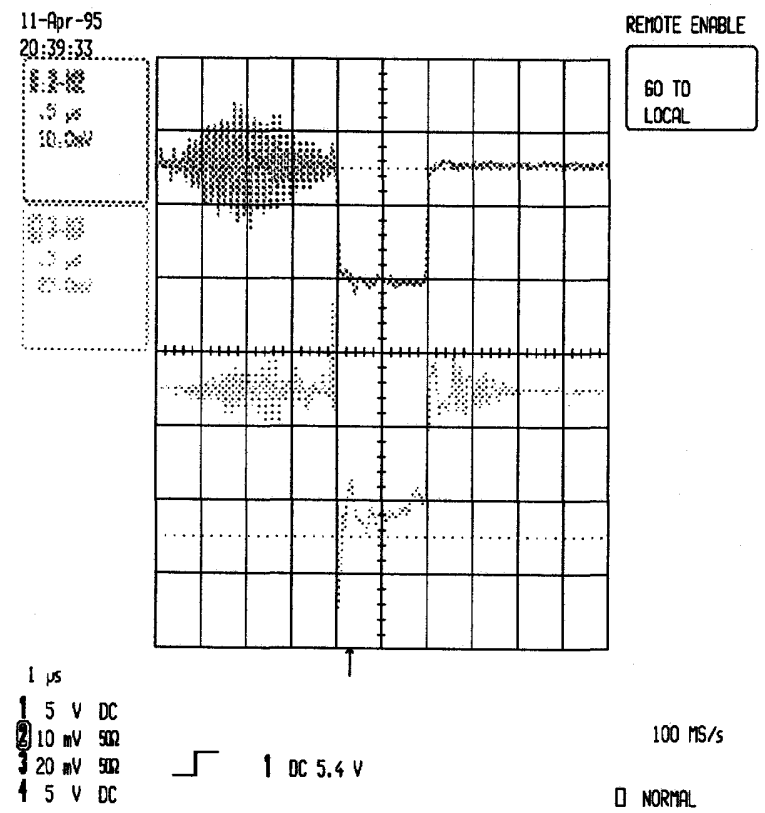

Figure E.3: Output of Monitor after Noise Subtraction 

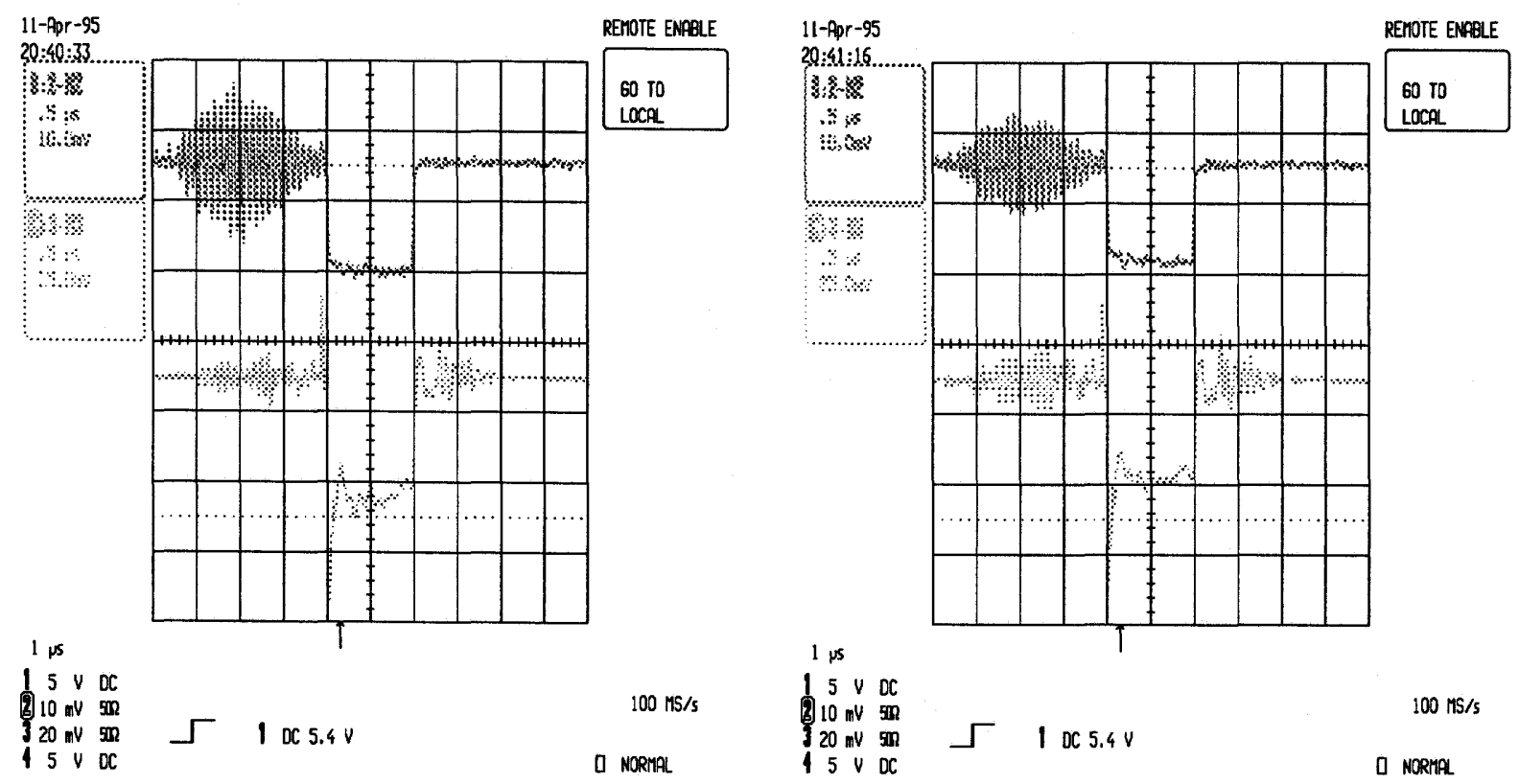

Figure E.4: Prebuncher Phase $9^{\circ}$

Figure E.5: Prebuncher Phase 49

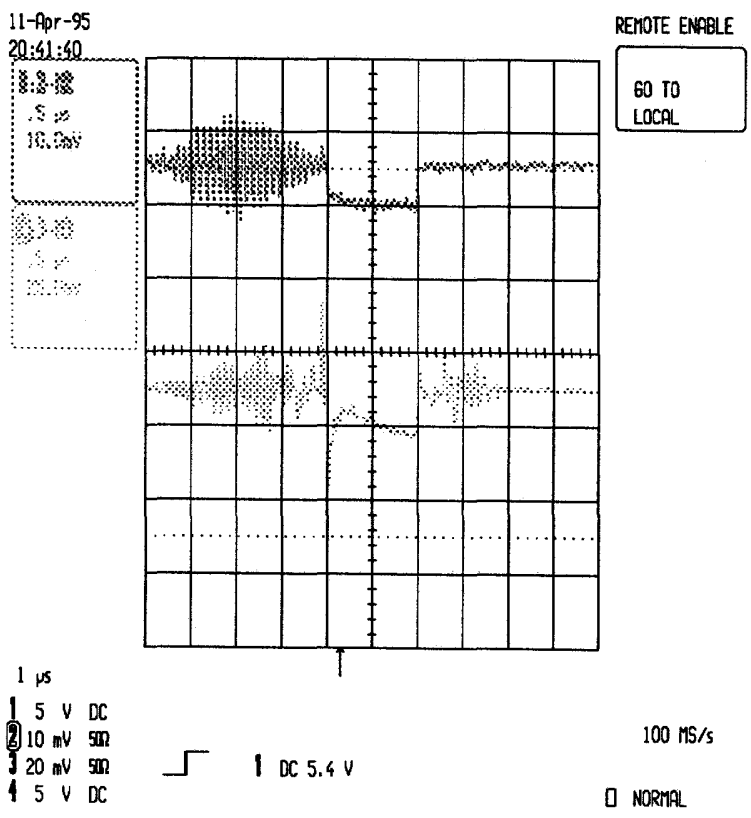

Figure E.6: Prebuncher Phase $69^{\circ}$ 


\section{References}

[1] Alpha Industries, Microwave Semiconductors, 1983.

[2] Andrew Corporation, Andrew Corporation Catalogue 33, 1986.

[3] J. Berk and R. F. Koontz, Injector, in The 2 Mile Linear Accelerator, edited by R. Neal, Benjamin, New York, 1968.

[4] J. Borer and R. Jung, CERN Publications 84-15, 438 (1984).

[5] K. L. Brown and et al., Proceedings of the 1961 International Conference on High Energy Accelerators, pp. 79-117, Brookhaven, 1961.

[6] P. Brunet and X. Buffet, Electron Injectors, in Linear Accelerators, edited by P. M. Lapostolle, North-Holland Publishing Company, Amsterdam, 1st edition, 1970 .

[7] J. H. Cupérus, Nucl. Instrum. and Methods. 145, 219 (1977).

[8] L. O. Dallin, High Duty Factor Monochromatic Extraction from EROS, PhD thesis, University of Saskatchewan, 1990.

[9] J. W. Eaton, Octave Documentation Guide, 1.0 edition, 1994.

[10] L. J. Geis, Transform Analysis and Filters, Prentice-Hall, Inc., Englewood Cliffs, N.J., 1989.

[11] D. A. Goldberg and G. R. Lambertson, AIP , 537 (1992).

[12] Haimson Research Corperation, $13 \mathrm{MeV}$ Injection Linear Accelerator Model HRC-735 Instruction and Maintenance Manual, 1980.

[13] T. Ieiri, A Bunch Length Monitor by Detecting Two Frequency Components of the Beam, in Proceedings of the 7th Symposium on Accelerator Science and Technology, Osaka, Japan, December 12-14 1989.

[14] G. Jackson, private communication, 1994.

[15] J. D. Jackson, Classical Electrodynamics, John Wiley \& Sons, Inc., New York, 2nd. edition, 1975.

[16] G. F. Knoll, Radiation Detection and Measurement, John Wiley \& Sons, New York, 2nd. edition, 1989.

[17] R. Kollath, Particle Accelerators, Sir Isaac Pitman and Sons Ltd., London, 1967. 
[18] R. E. Laxdal, Design of an Energy Compression System for the Saskatchewan Linear Accelerator, Master's thesis, University of Saskatchewan, 1980.

[19] M/A-COM, RF and Microwave Control Components, 1993.

[20] D. Mckay, private communication, 1994.

[21] Motorola, MECL Device Data, 1987.

[22] E. Persico, E. Ferrari, and S. E. Serge, Principles of Particle Accelerators, W. A. Benjamin, Inc., Amsterdam, 1968.

[23] C. L. Phillips and J. H. T. Nagle, Digital Control System Analysis and Design, Prentice-Hall, Inc., Englewood Cliffs, N.J., 1984.

[24] D. M. Pozar, Microwave Engineering, Addison-Wesley Publishing Company, Inc., New York, 1990.

[25] R. E. Shafer, Beam Position Monitoring, in AIP Conference Proceedings 212: Accelerator Instrumentation, edited by E. R. Beadle and V. J. Castillo, New York, NY, 1990, American Institute of Physics.

[26] J. C. Sheppard, J. E. Clendenin, M. B. James, R. H. Miller, and M. C. Ross, IEEE Transactions on Nuclear Science NS-32, 2006 (1985).

[27] Texas Instruments, Linear Circuits Data Book, 1989.

[28] H. Wiedemann, Particle Accelerator Physics, Springer-Verlag, Berlin, 1993. 